\title{
Persons and their Minds
}

Towards an Integrative Theory

of the Mediated Mind

Svend Brinkmann

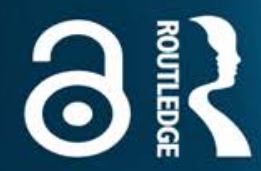




\section{Persons and their Minds}

"Persons and their Minds is a remarkably elegant, deeply scholarly and integrative proposition for a new 'hybrid psychology'. Drawing on classical and current debates in philosophy, cognitive sciences, social sciences and psychology, Svend Brinkmann proposes an original theory of persons, with their minds as sets of practices and dispositions, mediated by their brain, bodies and social worlds. Written with simplicity and humor, this monograph brings much-needed theoretical and epistemological clarifications - demonstrating why, among other things, people's actions cannot be explained by brain mechanisms. This groundbreaking work offers a new basis for understanding psychopathology and paves the way for an integrative psychology of people's everyday life in society."

-Professor Tania Zittoun, University of Neuchâtel, Switzerland

"Brinkmann's book provides the long-sought integration of the various strands of psychology and will be the starting point of the unification of the sciences of the mind."

- Professor Rolf Reber, University of Oslo, Norway

Today's approaches to the study of the human mind are divided into seemingly opposed camps. On one side we find the neurosciences, with their more or less reductionist research programs, and on the other side we find the cultural and discursive approaches, with their frequent neglect of the material sides of human life. Persons and their Minds seeks to develop an integrative theory of the mind with room for both brain and culture. Brinkmann's remarkable and thought-provoking work is one of the first books to integrate brain research with phenomenology, social practice studies and actor-network theory, all of which are held together by the concept of the person.

Brinkmann's new and informative approach to the person, the mind and mental disorder give this book a wide scope. The author uses Rom Harré's hybrid psychology as a metatheoretical starting point and expands this significantly by including four sources of mediators: the brain, the body, social practices and technological artefacts. The author draws on findings from cultural psychology and argues that the mind is normative in the sense that mental processes do not simply happen, but can be done more or less well, and thus are subject to normative appraisal.

In addition to informative theoretical discussions, this book includes a number of detailed case studies, including a study of ADHD from the integrated perspective. Consequently, the book will be of great interest to academics and researchers in the fields of psychology, philosophy, sociology and psychiatry.

Svend Brinkmann is Professor of Psychology in the Department of Communication and Psychology at the University of Aalborg, Denmark. His primary research areas are general psychology and qualitative methods. He is currently Co-director of the Center for Qualitative Studies at the University of Aalborg and member of the Center for Cultural Psychology. 
The series Cultural Dynamics of Social Representation is dedicated to bringing the scholarly reader new ways of representing human lives in the contemporary social sciences. It is a part of a new direction - cultural psychology - that has emerged at the intersection of developmental, dynamic and social psychologies, anthropology, education and sociology. It aims to provide cutting-edge examinations of global social processes, which for every country are becoming increasingly multi-cultural; the world is becoming one "global village", with the corresponding need to know how different parts of that "village' function. Therefore, social sciences need new ways of considering how to study human lives in their globalizing contexts. The focus of this series is the social representation of people, communities, and - last but not least - the social sciences themselves.

In this series

\section{Cultural Realities of Being}

Abstract ideas within everyday lives

Edited by Nandita Chaudhary, S. Anandalakshmy and Jaan Valsiner

Interaction, Communication and Development

Psychological development as a social process

Charis Psaltis and Anna Zapiti

\section{Engaging Violence}

Trauma, memory and representation

Edited by Ivana Maček

\section{Rethinking Creativity}

Contributions from social and cultural psychology

Edited by Vlad Petre Glăveanu,Alex Gillespie and Jaan Valsiner

\section{Optimizing the Self}

Social representations of self-help

Ole Jacob Madsen

\section{Imagination in Human and Cultural Development}

Tania Zittoun and Alex Gillespie

\section{Representing Development}

Pasts, presents and futures of transformative models

Edited by David Carré, Jaan Valsiner and Stefan Hampl

\section{Persons and their Minds}

Towards an Integrative Theory of the Mediated Mind

Svend Brinkmann 


\section{Persons and their Minds}

\section{Towards an Integrative Theory of the Mediated Mind}

\section{Svend Brinkmann}

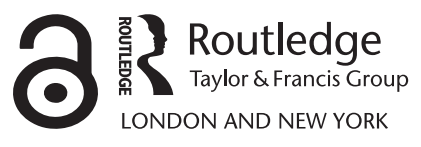


First published 2018

by Routledge

2 Park Square, Milton Park, Abingdon, Oxon OXI4 4RN

and by Routledge

7II Third Avenue, New York, NY I00I7

Routledge is an imprint of the Taylor \& Francis Group, an informa business

(C) 2018 Svend Brinkmann

The right of Svend Brinkmann to be identified as author of this work has been asserted by him in accordance with sections 77 and 78 of the Copyright, Designs and Patents Act 1988.

The Open Access version of this book, available at www. taylorfrancis.com, has been made available under a Creative Commons Attribution-Non Commercial-No Derivatives 4.0 license

Trademark notice: Product or corporate names may be trademarks or registered trademarks, and are used only for identification and explanation without intent to infringe.

British Library Cataloguing-in-Publication Data

A catalogue record for this book is available from the British Library

Library of Congress Cataloging-in-Publication Data

Names: Brinkmann, Svend, author.

Title: Persons and their minds : towards an integrative theory of the mediated mind / Svend Brinkmann.

Description: New York : Routledge, [2017] | Series: Cultural dynamics of social representation | Includes bibliographical references.

Identifiers: LCCN 2017009590 | ISBN 978II38653696 (hardcover) | ISBN 978 I 3 I 5623658 (ebook)

Subjects: LCSH:Thought and thinking. | Mind and body. | Sociology.

Classification: LCC BF44I .B755 2017 | DDC I28/.2-dc23

$\mathrm{LC}$ record available at https://lccn.loc.gov/20I7009590

ISBN: 978-I - | 38-65369-6 (hbk)

ISBN: 978-I-3 I5-62365-8 (ebk)

Typeset in Times New Roman

by Apex CoVantage, LLC 


\section{Contents}

Series editor's preface

vii

Foreword

Introduction

1 The concepts of mind and person 8

2 The mind and the brain $\quad 34$

3 The mind and the body $\quad 58$

4 The mind and society $\quad 75$

5 The mind and things $\quad 94$

6 The mind between the brain and culture 107

7 Persons and disordered minds 116

$\begin{array}{ll}\text { References } & 129\end{array}$

Index 137 
$\because$ Taylor \& Francis

Taylor \& Francis Group

http://taylorandfrancis.com 


\section{Series editor's preface}

"The mind has no breakfast"

- but we have a new theory!

It is time to set basic ideas into their rightful places - after science journalists have frantically displaced them for the sake of creating a public interest havoc. Topics like "mind", "self" and "brain" are of that kind. The promises made about the almighty power of the neurosciences have become a discourse form that makes a link with serious science in its popular representation - and hence ends up in a fashion show of multi-coloured brain images that hide the reality of basic sciences about the mind actually cover (Werbik \& Benetka, 2016). This reality - or maybe best called counter-representation of socially over-represented knowledge domain - needs to be revealed before the "bubble" of contemporary neurosciences "bursts" and we again are facing the nakedness of the Brain-King in front of all of us, the mental miserables.

Although the critique of neurosciences - or better - publicized travesties of the brain sciences - is a theme in this book, the reality of the constructive offering that the reader can encounter here is elsewhere. Brinkmann replaces both brainbased and mind-based reductionist accounts of the human psyche by a third alternative - structured dynamic processes in-between. The "in-between" involves two interspersed domains - that of the relation of the body (and the brain) with its environment, and that of the person (with her mind) with the society (and its social norms for "the mind" - patriotic, feminist, masculinist or just plainly "pathological"). The idea - that there is no way for the mind to have a breakfast - like we normal persons do easily every morning - is the crucial starting point to create a new theory of the mind-in-life and to maintain psychological theory as a framework that goes beyond any version of reductionism.

Svend Brinkmann is in the best position to systematically sieve through the various representations of the mind, and the misplaced precision on which claims of contemporary neurosciences are built. He is both a philosopher and psychologist - a rare combination in our contemporary world. In this book he undresses the excessive garments of the neurosciences in his systematic, careful 
and silently humorous manner that is his intellectual trademark across all of his books (e.g. read Brinkmann, 2011; 2013; 2017).

This book is a careful move towards a new kind of psychology. It is rooted in the ideas of Ludwig Wittgenstein, John Dewey and Rom Harré. Of these three, it is the latter two who have made constructive suggestions for psychology to develop a humanly adequate general framework. Brinkmann takes up their challenge and provides a new solution. It could perhaps be called a Theory of Human Dynamic Normative Intentionality. What is proposed in this book is a theory that deals with human life experiences as these are cultural in their nature. The proposed theory is a major contribution to our contemporary area of cultural psychology a burgeoning area of research uniting developmental and social psychology with anthropology, sociology and history - but which nevertheless has suffered in its history of past three decades from absence of unifying general theories and lack of methodology.

Brinkmann's theory is of such a unifying kind. It emphasizes the dynamic nature of the personal making up of one's mind - culturally, through signs. The beneficial background of John Dewey is clearly behind his way of building the dynamic focus of human subjectivity through semiosis. The normative focus in the theory is based on the advancement of the focus in psychology introduced by Rom Harré, but left without the elaboration of implications that the notion of multiplicity of grammars brings to our understanding of the human mind. Brinkmann makes that bold step beyond Harré - and with productive outcomes. In his own words:

If only one conclusion is to be drawn from this book's chapters, then it should be that psychology needs more than one form of understanding and more than one level of analysis. The brain alone is not enough, nor is the body, culture or materiality. This is why the attempts at monopolization that we have seen in the history of psychology have been unproductive when they seek to dispel one of the two halves of psychology that were outlined in the beginning of the book - the nomic or normative. Against this background we have to conclude that both psychologies - or rather both sides of psychology - are relevant and indispensable within its respective domain. But this is where the problem comes in: can we have both of these psychologies at the same time?

Brinkmann answers the last question in the affirmative. The notion of multiple grammars fits here - human beings are constantly constructing experiences through signs, organized by the norms of multiple grammars at the same time. The result is sometimes clarity, at other times confusion. The latter was Ludwig Wittgenstein's diagnosis of psychology as science in the 1950s. Unfortunately Wittgenstein had no intention for getting out of the confusion - his "language games" did not provide constructive solutions. Brinkmann does.

How does he arrive at a productive solution? By accepting that the intentional action by human beings - the idea that Franz Brentano posited in the 1870s but 
failed to elaborate in the dynamic realities of living - requires the use of multiple normative orders. These are "grammars" - that organize human experiencing. The reader will find these grammars the framework that guides intentional actions. Intentionality is always finding its expressions through the grammars-guided production of experiences. And the grammars themselves are human inventions. Human psyche regulates itself through creating normative systems for oneself, and then acting as if negotiating the borders of such normativity (Valsiner, 1999).

The result of Brinkmann's theoretical synthesis is a hybrid theory - inclusive of biological, psychological and social facets, and not prioritizing any of the levels. What links the levels is the agentive role of the human being who seeks cultural solutions for biological necessities and feels annoyed if these are not available. In Brinkmann's own final synthesis:

... the mentalistic or mindist approach, which regards the mind as a mental world in its own right, which can be discussed independently of neural, physical, social and technological conditions. Such a world does not exist. The mind is not a "world", nor is it a "thing", but rather a range of skills and dispositions that exists in the world and which is identified on the basis of a normative order of human activities and practices.

The reader of this book is up to the experience of seeing a new theoretical system in the making. For Brinkmann psychology is not "in crisis" but a productive arena for new value of careful philosophical thinking in the solving of basic problems of human being. I think we have much to learn from him.

Jaan Valsiner

Aalborg, January, 2017

\section{References}

Brinkmann, S. (2011). Psychology as a Moral Science: Perspectives on Normativity. New York: Springer.

Brinkmann, S. (2013). John Dewey: Science for a Changing World. New Brunswick, NJ: Transaction Publishers.

Brinkmann, S. (2017). Stand Firm: Resisting the Self-Improvement Craze. Cambridge: Polity Press.

Valsiner, J. (1999). I create you to control me: A glimpse into basic processes of semiotic mediation. Human Development, 42, 26-30.

Werbik, H. \& Benetka, G. (2016). Kritik der Neuropsychologie. Giessen: Psychosozial Verlag. 
$\because$ Taylor \& Francis

Taylor \& Francis Group

http://taylorandfrancis.com 


\section{Foreword}

In a certain sense, this book has an almost impossible ambition: To articulate a general theory about persons and their minds in a single book. There are so many classical and modern ideas about what the human mind is that a full explanation and discussion could fill countless books. A book about the mind is therefore always a partial contribution to the existing corpus of ideas that needs to be understood in the historical and cultural context in which it is written. There is so much disagreement about what the mind is, how it should be studied and whether it even exists that you would end up with a very fragmented book on the subject if you did not find a specific perspective from which to analyze it. In this book I present just such a perspective; it does not, therefore, represent a neutral introduction to the subject. The book only indirectly provides an overview of theories and studies of the mind, and is, in a more direct sense, a specific theory of the mind, which also seeks to synthesize many diverse theories under the umbrella term of cultural psychology. One of the book's ambitions is to show that many of these theories can coexist.

A first edition of the book was published in Danish in 2009, entitled The mindbetween synapses and society, by Aarhus University Press. The manuscript has been thoroughly revised and expanded for the English-language version. I wish to thank Aarhus University Press for giving me full permission to develop the Danish manuscript into a longer and more comprehensive book in English, and Asher Ariel for help with translations. I would also like to thank Cecilie Eriksen, Jacob Klitmøller, Casper Larsen and anonymous reviewers for many helpful and relevant comments on the original manuscript. I am particularly grateful to Jaan Valsiner for inviting me to write a volume for the book series on the Cultural Dynamics of Social Representation. In recent years, Jaan has been an incredible source of inspiration for me as a scholar and colleague. As always, my greatest thank you goes to my family - Signe, Jens, Karl and Ellen - for coping so well with me through the years. 
$\because$ Taylor \& Francis

Taylor \& Francis Group

http://taylorandfrancis.com 


\section{Introduction}

This book is about a phenomenon that on the surface seems like the most obvious and relevant thing for people, while at the same time it is perceived by many as one of the most mysterious and inaccessible to us - and this in spite of recent advances in modern science: the mind. On the one hand, the fact that we have a mind (whatever that means, which is the central question of this book), or that we are psychological beings, is a matter of course for us since we, as long as we are alive, have a mental life: We think, feel, act and dream, along with a host of other things that would reasonably be regarded as psychological processes. The fact that my limbs can move in specific ways is an expression of my physiological and anatomical characteristics, but the fact that they are moving because I want to achieve a specific objective with that movement (like writing a book by moving my fingers on the keyboard) is the expression of specific psychological capacities within me. Everything we do falls within the field of psychology (although not exclusively within this field, since neighbouring disciplines like sociology and anthropology are also relevant when studying human action), and the fact that we can do things intentionally is what justifies the assertion that we have a mind. That is why the mind, at first glance, is entirely obvious and continuously present for us.

On closer inspection, however, the mind has proven extremely difficult to examine scientifically, and the sciences that deal with the mind (psychology and psychiatry in particular) are full of controversial views that certainly are not obvious, including the notion that the mind does not even exist (as argued by eliminative materialists), that it can be reduced to processes in the brain (according to some neuroscientific theories), that it can be dissolved into social relations in its entirety (as some social constructionists have claimed), or that individuals do not have any privileged insight into their own mental and psychological lives (as the behaviourists say). In the book we will see examples of these and many other challenging, controversial and hopefully exciting ideas about the mind, which if it exists - can reasonably be said to be the most complex phenomenon in the universe.

People are greatly interested in theories of the mind around the world. Psychology is one of the university subjects with the most applicants in many countries, 
and psychological knowledge is used almost everywhere in society where people are involved: in education, parenting, business, marketing, politics and many other fields (Brinkmann, 2008a). Popular science books on all sorts of psychological phenomena, from self-esteem to psychopathy, are being read like never before, and self-help books that encourage the use of psychological techniques like coaching, self-improvement and therapy, are among the best-sellers (Brinkmann, 2017). Much of the current discourse on the mind is marked by almost religious language, which recommends that you "find yourself" and "develop yourself", but, almost paradoxically, nearly as much of the discourse is marked by a scientific way of thinking. Books about brain research and the effects of genetics on behaviour are read with great interest today and they stand side by side on bookstore shelves with New Age-inspired self-improvement literature. We have many different conceptions of the mind these days, which are all far from compatible, but which affect the way we understand ourselves to varying degrees. This book seeks to create a language of the mind that enables the integration of some of the actual portrayals of the mind that can and should be integrated, but which at the same time allows for rejection of the numerous portrayals that are unproductive, distorted or patently wrong.

One false portrayal depicts the mind as a disembodied object, which is based in, or which can be directly identified with, the human "inner world". This portrayal plays a major role, for example, when we imagine that we each possess an "inner self" or an "inner potential" that can and should be realized both at work and in our private lives. Whereas this portrayal is associated with some semi-religious conceptions, where psychology acts as a kind of secularized religion and where the Christian notion of a disembodied soul is merely replaced by a psychological notion of a disembodied self, another equally false portrayal is more often associated with modern science. This is the portrayal of the mind as simply an aspect of the brain. In recent years, confidence levels in the results of brain research and their importance for psychology and psychiatry have been extreme (see Chapter 2). This book in no way rejects modern brain research, but raises serious doubts about the current imperialistic colonization of brain research that we are witnessing today in the fields of psychology, psychiatry, culture, science and even ethics and theology. One goal of this book is to put brain research in its rightful place among the many types of research that are needed to understand the mind. But it belongs in only one place among the many.

This book is about the human mind as a product of nature/biology and culture/ society, respectively. My own scientific background is in philosophy and psychology, which literally means the science (logos) of the mind (psyche). There has been a distinct ideological conflict between perspectives from biology and society - or between the brain and culture - in scientific psychology in recent years. The central message of the book is that there is no contradiction between the biological investigation of the neural basis for the mind (brain) and the societal imprinting of the mind (culture and social practices). Consequently, I try to develop a comprehensive and coherent understanding of the mind that is explicitly multidisciplinary, 
and which implies that neuroscientists and cultural anthropologists, for example or scientists and humanists more generally - should not be regarded as being in opposition to one another, but should instead be seen as each having a grasp on the two sides of the hybrid phenomenon that we call the mind (this is argued in Brinkmann, 2011b; 2012, which together comprise some of the background research for this book). Although my point of view is broad, inclusive and pluralistic, the book is not neutral in its position - far from it. The book attempts to show that two approaches to the mind in particular are flawed: The first is the mentalistic approach, which says that the mind is a world in its own right and that it can be studied independently from the brain, body and culture. Mentalism is the view that there are self-contained mental states (e.g. beliefs or emotions) that underlie human actions - that there is a dualism between the mind and the world, and that there is an external relation between mental states and observable actions, such that private mental states cause the actions that other people can see.

Mentalism can be traced back to the philosopher René Descartes (1596-1650), who believed that the mind was a thinking thing - an independently existing metaphysical realm - which was connected to a living organism almost only by chance. For Descartes the mind was a substance, a kind of medium for the metaphysical soul. Although there are practically no psychologists and philosophers who adhere to Descartes' form of mentalism today, mentalism has nevertheless survived in many newer guises and it is often seen, paradoxically enough, among researchers who explicitly criticize Descartes - certain materialists, for example, who believe that everything in existence must be understood in terms of physical matter. Some of them can be criticized for being "neural Cartesians", since they may well have killed Descartes' idea of the mind as an independent metaphysical realm, but at the same time they have retained his idea that the psychological realm is an inner, private world, which is now merely believed to exist in the brain and not as a substance of the soul (Coulter \& Sharrock, 2007, p. 11). Other psychological researchers in the cognitive science tradition (Gardner, 1987) also perpetuate mentalism by operating with a theory of the mind as inner, mental representations that are sought to be understood in separation from the world they represent.

The second overarching approach that this book is critical of is the impending reductionism that we have seen in recent years in psychology, where brain research in particular is experiencing rapid growth. In very general terms, the reductionist approach argues that " $\mathrm{A}$ is nothing but B". Reductionism may well be a legitimate approach in certain fields. For example, I think it is reasonable to make the following reduction: "Crystal healing is nothing but an activity that exploits the placebo effect". But when it comes to the relationship between the brain and the mind in general, I think it is a misguided approach. I would argue, for example, that one would be mistaken to say that "psychological processes are nothing but electricity in a pattern of synapses," although I do not doubt that neural activity is a necessary condition for the existence of psychological processes as such.

My pluralistic and anti-reductionist perspective has not simply been conjured up out of thin air, but is mainly based on the thinking about the mind that has 
developed in the wake of Ludwig Wittgenstein's (1889-1951) philosophy. Wittgenstein, who was undoubtedly among the most important thinkers of the twentieth century, dealt intensively with the philosophy of psychology and was highly critical of the psychology of his day because he did not think that its experiments answered the basic conceptual problems that plagued the science. This book is not a thorough interpretation of Wittgenstein's philosophy, nor of the specific part of his philosophy that relates to the mind, but is instead based more on the insights that some of Wittgenstein's more recent successors have developed in the fundamental thinking about the mind. ${ }^{1}$ I would highlight three researchers in particular that are crucial to the perspective developed in this book: Peter Hacker, Rom Harré and Jeff Coulter. ${ }^{2}$ They come from a philosophical, psychological and sociological research environment, respectively, which is completely in line with the multidisciplinary approach of this book. In recent years, all three of them have used Wittgenstein's thinking as a springboard, in part to criticize mentalism and mind-brain reductionism, but also to develop more constructive suggestions for what modern thinking about the mind should look like. Harré and Coulter have also been involved in empirical research, and Harré in particular has been constructive and not just critical; he has developed what he calls a "hybrid psychology", which, like this book, seeks to integrate the neural and the cultural in an understanding of the mind that does not end in a case of reductionism (Harré \& Moghaddam, 2012).

In addition, the American pragmatist John Dewey (1859-1952) and the Russian psychologist Lev Vygotsky (1896-1934) are important sources of inspiration for this book's arguments, and the French phenomenologist Maurice Merleau-Ponty (1908-1961) also plays a certain role. The book, like its sources of inspiration, is multidisciplinary and the reader should therefore not expect a book about psychology in the traditional sense. The book is more precisely about the mind, where a psychological angle is just one of many, and where philosophy in particular is also brought into play. I give many examples from psychology throughout the book, but the book is not intended as an introduction to psychology's many areas, such as clinical psychology and personality psychology. Rather, it is an attempt to define what the mind is, and it thus represents a philosophically inspired general psychology, closely aligned with cultural psychology, which I see as the most sophisticated and philosophically mature version of contemporary psychology (Valsiner, 2014). I share with many cultural psychologists a distrust of a reification of the mind and also a wish to put the concept of the person at centre stage in the discipline of psychology. The book is thus called Persons and their Minds to underline the fact that minds are skills and dispositions of persons, the latter being psychologically fundamental (Harré, 2016).

The book's basic idea, to which I continually return and reveal the implications of, is that the mind as a concept refers to a range of abilities and dispositions of living human beings and not to any particular "thing" attached to persons. A widespread metaphysical preconception is that what something "is" is identical to what it is "made of" (Coulter \& Sharrock, 2007, p. ix). This may apply when 
we talk about phenomena like water, which we all know is "made of" water molecules, each of which consists of two hydrogen atoms and one oxygen atom. But if we transfer this metaphysical concept to the mind, we can easily end up saying strange things such as "the mind is the brain." If the mind, however, is not a thing but rather a range of abilities and dispositions (to think, feel and act), then we avoid this conclusion because these abilities cannot be attributed to the brain in a meaningful way. The brain does not think, nor does it feel or act. Only human persons, or creatures that act like humans in required ways such as some non-human animals, can meaningfully be said to think, feel and act. Naturally, they could not do so without their brains, but that does not mean that their brains are performing these activities. Similarly, you cannot drive a car without an engine, but that does not mean that it is the engine that is driving. The book attempts to describe some consequences of this insight, which seems so simple at first glance.

The idea of this book is that we need a number of very different levels of analysis in order to understand our abilities and dispositions - and thereby understand our mind. I highlight the brain, the body, society's social practices and things/ technologies as being relevant analytical dimensions for understanding the human mind. None of these levels can stand alone, and, in principle, none of them can be dispensed with either. If you imagine an everyday phenomenon such as a group of schoolchildren building a bridge in order to learn practical mathematics, there is a huge amount of psychological processes involved. The most important among them is that the children have the intention to build the bridge. But they can have such an intention only provided that they have brains that function in certain ways. They also have to have bodies with which to act in the physical world and realize their intentions. They also have to be able to work together, so that when one of them acts, the others respond accordingly in a social context. The children are also standing on the shoulders of the traditions of mathematics, education, building bridges and cooperation, whether they are conscious of it or not. And, finally, the children are also in a world of things; these are in part physical things, including the materials that the bridge will be built from, with all of the possibilities and limitations that these things entail for the children's realization of their goal, but also things that have semantic content, such as the books that the children can consult to get ideas for the construction of the bridge. At first glance it seems to be a simple psychological event - wanting to build a bridge - but it turns out that it depends on a wide variety of conditions such as brains, bodies, social relationships and culture as well as physical objects. That is the main thesis of this book: that this complexity applies to the mind in its own right as well as to all mental phenomena.

The book begins with a chapter that provides some background knowledge about the key concepts of the person and especially the mind. In this context, I also touch on some of the history behind the ideas that underlie the contemporary investigation of the mind. This is probably the most difficult chapter, since it describes lengthy and complicated historical developments in a very condensed format. The reader may want to skip this chapter initially and then return to it 
after having read the remaining chapters. Next, I focus on the four analytical levels, which consist of the brain, the body, society's social practices and things and which also constitute the book's four central chapters (2, 3, 4 and 5). In Chapter 2, which discusses the brain, I work from the premise that the brain is a necessary organ for a psychological life to exist, but I argue at the same time that the mind cannot be said to end at the skull - and that the mind's location therefore cannot be restricted to the brain. Chapter 3 expands the boundaries of the mind, so that the psychological realm can also be said to include the body as a central component. In recent years, this has been investigated in particular by phenomenologists of the body, based on the work of Maurice Merleau-Ponty. But not even the body's skin forms the boundary for where the mind ends, because a complex psychological life also requires certain social practices and relations, certain forms of human sociality - or society in the literal sense ("society" comes from Latin societas "fellowship, association, alliance, union, or community," from socius "companion") - which contributes to shaping the abilities and dispositions that constitute the mind. Social practices, including family and community life, are the subject of Chapter 4 , which is based in particular on current theories in cultural psychology. Next, in Chapter 5, I argue that not even a pure, intersubjective sociality ("society") marks the boundaries of the mind, because to fully understand the mind we also have to include the things and technologies that surround us. Most mental abilities and dispositions are possible only because we as people avail ourselves of tools, artefacts and other material objects (everything from reading glasses and alphabets to personal computers), which therefore become essential for the sophisticated forms of mental life that we humans have developed. The book's perspective in this regard comes close to the point of view of modern theoretical psychology called externalism - which means that the mind, in a certain sense, is something "external" and does not exist solely inside people's heads (Noë, 2009). Man is a tool- and technology-using species, so the notion that we are cyborgs, whose lives depend on things and technologies, is not just a distant vision of the future. We have been this way since the Stone Age.

The argument, then, is that we should not look for a "boundary" within which the mind can be confined (not the skull, skin, community or material objects) because the mind - as a range of abilities and dispositions - is understandable only if we include every level: the brain, the body, society and things. The mind is "spread out" over all of these levels. A metaphor for the mind could be an onion that you can peel without ever finding the core. We cannot find the mind as a "thing" by peeling away analytical layers and, for example, ending up looking into the brain, because the mind is simply not a thing. But neither is it nothing, which is also an important point for the book's rejection of reductionism. The concept of the person emerges as the necessary connection point between the different layers. Mental phenomena belong irreducibly to persons and not to any of the layers or tools that persons use in living their lives. Chapter 6 summarizes the arguments that back this up and outlines a "hybrid psychology" with inspiration from Harré in particular, which, as a science, is intended to involve the brain, the body, society 
and things when the mind is investigated. The final Chapter 7 applies the theory to mental disorders and develops a way of thinking about the psychological problems that humans face from time to time in their lives.

\section{Notes}

1 For works that specifically deal with Wittgenstein's philosophical psychology, reference can be made to Budd (1989) and Harré and Tissaw (2005).

2 See (Bennett \& Hacker, 2003; Coulter, 1979; 1989; Coulter \& Sharrock, 2007; Hacker, 2007; Harré, 1998; 2002). 


\section{Chapter I}

\section{The concepts of mind and person}

When we reflect on the psychological concepts in our language, ordinary language should have, if not the last word, then at least the first word, as the philosopher John Austin said. In this context, it is interesting that the word "mind" is actually not very commonly used in ordinary language. When the term is used, it is often in a context where we are talking about people's difficulties. We can talk about people having "psychological problems" and we understand what is meant by "a fragile mind" (and of course "a strong mind" as well), but only rarely do we talk about the fact that "thinking is a mental process" or that "the mind can be confined to the brain". In that sense, both "mind" and "mental" are scientific and semi-technical words. In this book's perspective, this means that we should be wary when theorizing or constructing scientific models of "the mind". What is it exactly that we are theorizing about and constructing models of? This is particularly important when dealing with the sciences that examine human experiences and actions, because if the results produced by these sciences cannot be traced back to something that is literally meaning $f u l$ for the people who are the subject of the research, then there is reason to doubt the legitimacy and validity of the research altogether.

This differs from the natural sciences, where there is no requirement that theories about quarks and superstrings need to mean something to so-called "ordinary people". But if psychologists tell people that they (psychologists) have discovered that feelings are something completely different than what we thought they were, then there is good reason to doubt that it is actually feelings that these psychologists have discovered something about. Again, as mentioned in the introduction: the psychological realm is something that is "close" to us and something we are all familiar with, so there is a limit to how ground-breaking the "discoveries" made by the sciences that study the mind can actually be. That is not to say that you cannot discover anything new about the mind, but even Freud's (1856-1939) psychoanalysis, which was very revolutionary and shocking to many people's understanding of the self, had to draw upon terms taken from ordinary language ("feeling", "sexuality", "dream", etc.) in order to be formulated, and psychoanalysts have to use such terms extensively in the same way as the terms are used in everyday language for the theory to be at all meaningful. This is an absolutely fundamental point, and it means that the sciences of the mind and psychology 
must always have a perspective from "within" human existence - at least in part. There are limits to how far the sciences of the mind can be from recognizable and everyday matters (from what is sometimes referred to as "folk psychology") before these sciences are no longer about what they intended to be about at the outset. For example, it is perfectly legitimate and necessary to study the importance of the brain for the mind, but if this leads to psychological concepts and explanations from everyday life being replaced by more "scientific" neurological concepts and explanations, then you quickly end up in a situation where you are no longer talking about something psychological. As we shall see in the next chapter, this is the problem with what is known as "eliminative materialism", a philosophical position which, among other things, aims to replace our vocabulary from "folk psychology" with a more "scientific" vocabulary. The achievement of this aim would mean that, for example, instead of saying "it hurts" we should say "my C-fibres are stimulated". One of the problems with this is the fact that it is not interesting for the person who was hurt that his C-fibres are stimulated as such - rather, what is interesting is that his finger was hit by a hammer and it hurts!

However, terms like "mind" and "consciousness" are more common in everyday language than "psyche". The latter term is older and virtually synonymous with the term "mind" and I will use them interchangeably throughout the book. "Psychological state" can be translated into "state of mind", and the slightly outdated "sentiment" translates into "emotion", which is a more modern psychological term for what we call feelings in everyday language. The term "consciousness" is a bit different. "Consciousness" is probably more widespread in ordinary language than "mind" (for example in questions such as "is he conscious?"). There is a branch of philosophy that we call "philosophy of mind" in English, and this term is often translated into other languages as "philosophy of consciousness". In Danish it is thus "bevidsthedsfilosofi". The philosophy of consciousness has become a major and important area in international philosophy, and there is also great interest in consciousness research within the fields of psychology and neuroscience. But even though the concept of consciousness is closely related to the concept of the mind, it cannot replace it. The most obvious reason is that not all psychological phenomena are conscious, although there may be grounds for considering that they must potentially be conscious in order for us to be able to correctly classify them as psychological. All phenomena of consciousness are psychological, but not all mental phenomena are conscious, which means that the concept of the mind is broader than the concept of consciousness. The human ability to solve complex problems, for example, is an ability that can be said to belong to the mind (and thus rightfully studied by psychology), and this is true regardless of whether problem solving is done consciously or not. Not all forms of problem solving are conscious in the sense of involving experiences of certain qualities (or qualia), and the concept of problem solving is not an experiential concept, but a normative concept in that it is about solving problems correctly. Consciousness is not, however, a normative concept, but an experiential concept; it is about what we experience and what we are aware of. The distinction between the normative 
and the experiential disciplines of psychology is elaborated in further detail below. For the time being we can say that the term "consciousness" refers specifically to our awareness and our ability to perceive the world, whereas the term "mind" also refers to all sorts of other ways of being in the world that are not necessarily directly related to perception and experience, but which instead have to do with acting or thinking, for example.

A third term that I have already used, which is related to "mind" and "consciousness", is the "mental". One can talk about "the mental realm" as akin to "the mind". In principle, this book could have used this term in its title, but it is not as flexible, linguistically speaking, since it does not work very well as a noun (it is reasonable to speak of "the mind", but "mentality" doesn't really work all too well). That is why I ended up using the term "mind", which has the disadvantage of being somewhat abstract and technical, but which in turn is broad enough to capture what this book is really about.

Like so many other words, the term "mind" has a predecessor in the ancient Greek in the form of psyche. In Greek mythology, Psyche (from the Greek psukhe meaning soul or breath of life) is a goddess who is married to the probably more well-known god of love, Eros. The goddess Psyche personifies the soul and is depicted as a woman with butterfly wings. Psyche and Eros had a daughter together named Hedone, who is the goddess of pleasure. We have an interesting Greek family of gods representing the soul, love and pleasure, and the first-mentioned lent her name to the human mind or psyche. It is thought-provoking that pleasure is an offspring of the soul and love: It is love that gives rise to pleasure and not the other way round. Incidentally, however, Psyche was not born a goddess, but was a princess whose beauty could compare with Aphrodite's (the goddess of beauty).

For the Greeks, Psyche did not represent the soul in the Christian sense, nor is it equivalent to a modern understanding of the mind as the seat of thoughts and emotions. In Greek thought, such as that of the philosopher Aristotle (384-322 $\mathrm{BC})$, about whom we will hear more below, the psyche is practically a biological concept. The mind is spirit, but first and foremost a biological kind of spirit. The mind is the first principle of living things, so all living things - animals, plants, humans - have psyche in virtue of being alive. The Greek psukhe also means respiration, and when you "expire" (from Latin meaning to breathe out) literally, you cease to be alive. In Danish we say "udånde", which literally means that "spirit" goes "out". According to Aristotle, the human mind distinguishes itself from the minds of other animals and plants in that it is rational. We can think and talk because we can use concepts, and, as rational beings, we can act on the basis of more or less good reasons. We can do this because we are raised, educated and cultivated by our social circumstances; "polis andra didaske", said the Greeks ("the city is man's teacher"). Since the times of the Greeks, the belief has been that we, as psychological beings, belong to biology (nature) on the one hand, and to society (the city) on the other. This duality is also the focal point of this book. Persons have minds because they result from cultivated nature in "cities" (a broad term here standing for organized social relationships). 
Before human beings began investigating the mind scientifically by using various psychological methods, and before we started treating psychological disorders with psychotherapy and psychotropic drugs, other concepts and practices were relevant for what we now regard as the mind. A large portion of these concepts and practices were religious and were associated with what is conceived of as the soul in Christianity. We must be wary here of uncritically thinking that the Greek concept of psukhe, the Roman concept of anima and the Christian concept of the soul correspond to what we today call the mind (Danziger, 1997). There have been a number of crucial conceptual transformations in the course of history. A large portion of theories about the mind in modern psychology depend on a basic concept of an "inner world", but this is far from the Greek concept of the mind as a biological principle and from the Christian idea of a substance of the immortal soul.

As the philosopher Charles Taylor has argued, the modern idea of the psychological realm as an inner world of thoughts and feelings is "a function of a historically limited mode of self-interpretation, one which has become dominant in the modern West and which may indeed spread thence to other parts of the globe, but which has a beginning in time and space and may have an end" (Taylor, 1989, p. 111). Taylor thus relativizes the notion of the psychological realm as an inner world to a specific culture and historical epoch, the beginning of which can be approximately determined as the eighteenth century. Interpreting the psychological realm as an inner, private world is a historically and culturally specific notion, derived from the Christian conception of an immortal soul; this conception, however, also existed prior to Christianity, among certain ancient Greek philosophers, for example. But for Aristotle, the concept of the mind was something altogether different, and therefore it is difficult to believe that the historically changing discourses about "the psychological realm" have always been about the same thing. In certain respects there are also similarities among the concepts of the mind that have dominated in different epochs. Both the Christian conception of the soul and the modern conception of the mind, for example, have been involved in governing human life in a similar manner. In medieval society, people were to live their lives in a certain way in order to achieve salvation of the soul and avoid perdition, which was a process that could be controlled by the Christian clergy. In modern society, the goal of existence, according to some psychological self-help literature, has correspondingly been the realization of the psychological self, mediated by listening to and accepting what psychotherapists, coaches and other self-improvement experts have to say. It is not difficult to see structural similarities between the way the soul was involved in the pre-modern governance of human life and the way in which conceptions of the mind shape our lives today (Rose, 1999). For many of us, developing ourselves, our personalities or our minds is not a right but an obligation that is imposed on us in the educational system (when, for example, we have to develop our social and emotional skills) or at work (when we are sent to personal development courses or when we are coached by our managers) (Brinkmann, 2017). In this sense, conceptions of the mind are not only scientific but also 
political and woven into various societal power relationships, just as the religious conceptions of the soul have always been.

Kurt Danziger, an influential historian of psychology, argues that the modern idea of the psychological realm as a distinct research field with its own subject matter can hardly be said to have existed before the eighteenth century (Danziger, 1997, p. 21). Before that time, its existence was thought of in religious, ethical and medical terms, but not yet in psychological terms. In short, people did not think of themselves as having a mind in the modern sense. But in the eighteenth century, British empiricist philosophers, perhaps especially David Hume (1711-1776), started to regard the mind as a piece of machinery with Newton's mechanical physics as a scientific model. When this mechanical psychology was combined with the experimental practices for investigating the life of the soul, which were developed by German physiologists about 100 years later, we had, for the first time in history, isolated the mind in the modern sense, which academic psychology takes for granted today. Some very specific conditions had to be met in order for it to make sense to start regarding the mind as a scientific object, and there are indications that these conditions are in large part linked to the historical processes of individualization. Psychology did not emerge as a science of the individual mind until the individual entered into history as an independent figure and God, tradition and society took the backstage. This is why we also see that psychology historically spread most quickly in relatively individualistic Western cultures, and it is not until much later that more collectivist cultures like Arabic or East Asian societies have developed psychology, which they have often imported from the West despite the fact that they already had well-developed scientific disciplines in other areas. Our conceptions of the mind are always historically and culturally influenced, and sometimes we are not even talking about the same thing, although we may use the same word: the mind.

\section{Two main tracks in the history of ideas about the mind}

Even though I think there is reason to caution against reading the Western history of ideas uncritically, as if the changing ideas about the psyche/soul/mind have always been about the same thing, I will tentatively identify the two main tracks in thinking about the human mind that still influence our conceptions today. These main tracks can be called (1) the mind as consciousness and (2) the mind as rationality. The first track focuses on the mind primarily as a mechanism for experiencing the world, while the second track regards the mind as a designation for our ability to think and act rationally. These two tracks are still both very important in current psychological research: the consciousness standpoint underlies much of neuropsychology, where consciousness is often sought to be reduced to something neurological, whereas the rationality standpoint primarily underlies various forms of cultural psychology, and it is a version of this that I shall defend in this book. I should emphasize, however, that the two tracks are not mutually excluding in the sense that the former track denies that people can be rational or that the latter 
track denies that we experience the world. That is not the point, but it is, rather, to find the foundational principle of the mind in either consciousness or rationality.

\section{The mind as consciousness}

Figure 1.1 aims to provide an overview of the historical development of conceptual relationships (inspired by Hacker, 2007, p. 27).

We can trace the consciousness approach to the mind back to the father of Western philosophy, Plato (427-347 BC), because of his argument that a human being consists of a body and a soul, and that it is the soul that essentially is the human; this idea finds particular expression in the dialogue Phaedo. The notion that all human beings carry something immaterial within them, which is what individualizes them (i.e. that which makes the individual who he or she is), was of great importance for the originator of modern philosophy, René Descartes, who, in the first half of the seventeenth century, developed his dualistic theory of two essentially different metaphysical substances: the extended substance and the thinking substance (Descartes, 2002). The thinking substance is the human mind, which Descartes understood as consciousness. For Descartes, to think something is to have conscious awareness of something - an idea, as he called it, or a "mental representation", as many modern cognitive scientists call it.

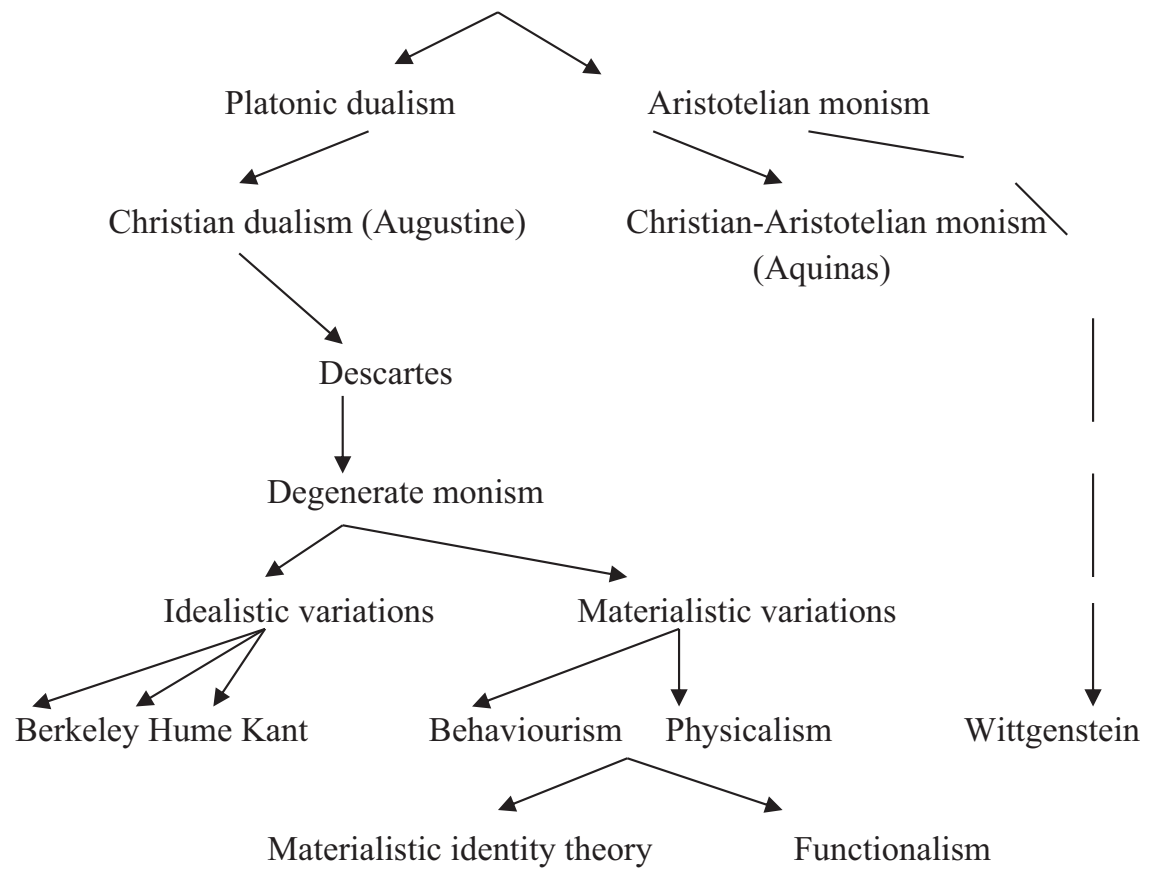

Figure I.I Conceptions of the human mind 
Descartes' concept of the "idea" is akin to Plato's, but whereas Plato saw ideas as referring to the basic cosmic forms that underlie and organize the world and appear to us phenomenally through our sense perception, Descartes' philosophical revolution consisted in attributing these ideas to man's inner self. In popular terms, he put the universe into the human mind and believed that the only things that people could ever perceive are the ideas that appear in their consciousness. An important transitional figure in the conceptual history from Plato to Descartes is the Church Father Augustine (354-430). For probably the first time in history, Augustine elaborated the very notion of the inner world that has been so central to modern man's self-understanding. Descartes could then subsequently assert that the inner world was populated with ideas. Consciousness is understood here as a kind of container in which there are certain objects, ideas or experiences that presumably correspond to phenomena in the outer world. But strictly speaking, we can only assume this - we can never know it - because when the only thing we can recognize is the content of our own consciousness, everything else (the outer world, other people's existence) becomes purely hypothetical, and we do not really have any reason to believe that anything other than the content of our own consciousness should exist. This absurd conclusion is called solipsism - the idea that one's own mind (understood as consciousness) is the only thing that exists.

Descartes had to perform some difficult moves in his arguments to try to avoid the solipsism to which his own starting point had led him. The existence of God was among the issues he argued for in the course of his philosophy, and his view led him, as mentioned, to conclude that there are two essentially different substances: the mental (consisting of ideas as experienced) and the physical (consisting of extended things). This dualism might seem strange to a modern, scientifically minded reader, but it is not as foolish as it is sometimes made out to be. For example, it is obvious that there is a difference between the physical Mount Everest, which is 8,848 metres high, and my mental conception or idea of Mount Everest, which is certainly not 8,848 metres high. My conception is not even 8,848 millimetres high, nor does it weigh 8,848 grams, because that is the case with this as well as all other mental phenomena: they do not extend in space nor do they have mass. There is no length, height or weight to my ideas and conceptions.

Descartes was right to respect this difference between material phenomena (e.g. Mount Everest) and psychological phenomena (e.g. my conception of Mount Everest). Some of the more recent and contemporary materialistic philosophers and scientists, by contrast, have a tendency to neglect this difference when trying to find the place in the brain where, for example, my "representation" of Mount Everest is supposedly located. They envision my idea of Mount Everest as being, in some sense, a small (mental) representation of the real Mount Everest in all its grandeur. The basis for Descartes' thinking about mental objects is that these have no spatial properties or other physical dimensions, although he was not completely consistent on this point when he nevertheless tried to confine the location of interaction between the body and soul to the pineal gland in the brain. Descartes' problem is how these two essentially different things can interact. Thinking 
about Descartes' problem can cause some serious bouts of headaches - and I speak from experience! And how do we even make room for phenomena of the mind and consciousness in a world that modern science has taught us is material? It was a great relief for me personally to discover that these unsolvable problems ought not to be resolved, since they are formulated in a way that makes them unsolvable from the outset. The very premise of the problems is wrong. The premise is that the psychological realm is a kind of consciousness container, and that psychological phenomena are objects in that container. In my opinion, we can resolve these problems only by dissolving them, in other words, by starting from quite a different premise, which is what Aristotle and much later Wittgenstein did - and what the rationality viewpoint of the mind more generally attempts, as is elaborated in this book.

Although Descartes, in his own way, was right to respect the difference between physical things and mental conceptions and did not seek to reduce the one to the other, he was wrong in that he interpreted this difference as a specific metaphysical difference that required the development of a dualistic world view. The difference in the way that we can meaningfully speak of the physical and the mental, respectively, is in reality - as this book will attempt to prove - a grammatical and not a metaphysical difference. It is thus not a difference that relates to the world's basic structure and being (metaphysics) but rather a difference that stems from the fact that we necessarily talk about different phenomena in different ways (grammar). The concept of being "grammatical" comes from Wittgenstein's philosophy, and grammar is to be understood as the network of rules and norms that determines which uses of language make sense and which do not (Wittgenstein, 1953). For example, it makes no sense to say that "the colour red weighs two kilograms", but not because red weighs a different amount or because we have not yet developed sufficiently sophisticated methods for weighing colours. The reason is simply that we cannot meaningfully attribute weight to colours - at least not when using the concept of colours that we currently avail ourselves of. To give another example, we can say that a book weighs 1 kilogram, but we cannot ask how much its contents weigh. If its content is weighty, it is only a figure of speech; the fact that the plot of a novel does not weigh anything is not a deep metaphysical insight. This "insight" does not require that we develop a dualistic metaphysical perspective about the difference between tangible and intangible things. More modestly, it requires that we become aware that different linguistic or grammatical rules apply when we talk about a thing's physical properties and its abstract properties, respectively.

We will see that many of the problems that the sciences of mind have faced over the course of history in fact stem from grammatical problems in the sense of Wittgenstein, rather than being empirical problems. What it comes down to, if we are to make progress in developing knowledge of mind, is thus not merely conducting new experiments and other empirical studies. Of course these are also necessary, but if we neglect the basic work, which is to clarify first what our concepts about the psychological realm really mean, then we risk conducting thoughtless or even 
meaningless studies. In his major work, Philosophical Investigations, Wittgenstein expressed it this way in the very last lines, which deal with psychology's status as a science:

The confusion and barrenness of psychology are not to be explained by calling it a "young science"; its state is not comparable, for instance, in its beginnings. (Rather with that of certain branches of mathematics. Set theory.) For in psychology there are experimental methods and conceptual confusion. (As in the other case, conceptual confusion and methods of proof.)

The existence of the experimental method makes us think that we have the means of solving the problems that trouble us; though problem and method pass one another by.

(Wittgenstein, 1953, p. 232)

Problem and method pass one another by in the science of the mind, according to Wittgenstein, because the problems are (at least primarily) conceptual, while the dominant methods are experimental (and typically seek to demonstrate a statistically significant difference between two populations). A great deal of experimental psychological research is simply conceptually naive and sometimes outright tautological. To say that something is tautological means that it does not prove or disprove empirical hypotheses, but instead is built on unrecognized conceptual relations. For example, an experimental study on the relationship between a stimulus such as an "unexpected situation" and the human response of "surprise" is tautological because surprise is defined as what one feels in unexpected situations (Smedslund, 2008). If such a psychological experiment, contrary to expectations, were to show that there is no connection between these two variables, then we would not revise our hypothesis about a real relationship but instead look for an error in our methods of measurement. The fact that we would react like this is generally an indication that we are dealing with a conceptual, rather than an empirical, relationship. One goal of this book is to conduct a conceptual investigation of the mind in order to avoid spending time on such pseudo-scientific studies.

In addition to Descartes' dualism between the mental and the physical, another of his dualisms has proved particularly persistent in Western thinking about the mind, and that is the dualism between the inner and the outer. If we commit ourselves to the notion that all existing objects can be localized in space, and if we acknowledge that thoughts, feelings and ideas cannot be located in the outer world, then we are obliged to invent an inner world where these phenomena can be placed. It is for this reason that the dominant depiction of the mind since Descartes - in science as well as in "folk psychology" - has been one that conceptualizes the mind as an inner world. This depiction is often coupled with a belief that the "things" that can be confined to the inner world are conscious experiences - and thus we have the main assumptions behind the idea of the mind as consciousness. ${ }^{1}$ Some consciousness researchers actually speak of consciousness as a "Cartesian Theatre" (Baars, 
1997). The mind is understood here by analogy with a stage on which conscious phenomena appear, and, regardless of the scientific progress that has been made since Descartes, it is plain to see that the mentalistic and reifying conception of the mind has been maintained.

Many modern consciousness researchers regard themselves as materialists and therefore cannot accept Descartes' idea of two essentially different metaphysical substances, only one of which is material. Instead, they argue that consciousness, as a "Cartesian Theatre" or the like, must have material dimensions like everything else in the universe, which means that it must be an aspect of the brain. They therefore attribute psychological states and conscious experiences to the brain, which as such comes to look like a materialist version of Descartes' "thinking thing" (see Bennett \& Hacker, 2003, pp. 68-70, for numerous examples of this kind of Cartesian materialism). In the next chapter we will elaborate on why it is problematic to attribute psychological properties to the brain (e.g. that it experiences, reasons, chooses, etc.). At the moment it is enough to observe that we could easily end up adopting this view as soon as we accept the premise that the mind is essentially defined as consciousness. The argument goes roughly as follows: Descartes was right in that conscious experiences form the core of the psychological realm; however, these experiences cannot (as he mistakenly believed, according to contemporary scientists) be attributed to a metaphysical other world (metaphysical dualism is rejected here); therefore, conscious experiences and thus the psychological realm must be properties of the brain (the dualism between the inner and the outer world is thus retained).

We can characterize the philosophers who reject Descartes' metaphysical dualism but accept his inner-outer dualism as "degenerate monists" (see figure above) (Hacker, 2007, p. 27). They are "degenerate monists" because they accept most of Descartes' premises, but they deny the existence of one side of his metaphysical dualism. Monism is the viewpoint that there is only one metaphysical substance in the universe, for example, that everything in existence is fundamentally composed of physical matter in motion. There are two types of degenerate monists: (1) the idealistic type (which is rare today), which includes the empiricist theories of Berkeley and Hume and (on some interpretations) Kant's transcendentalist theory, and (2) the materialistic type (which is dominant today), which includes behaviourism and the various forms of physicalism that will be discussed in the next chapter (see again the figure above).

The idealistic monists started from the premise that Descartes was right in saying that the only thing that we stand in epistemological relation to (and thus can have knowledge of) is the content of our own consciousness. Bishop George Berkeley (1685-1753) drew the logical conclusion from this, claiming that, consequently, only the contents of consciousness - or ideas - exist (hence the term "idealism"). "Esse est percipi" said Berkeley: To be is to be perceived by a consciousness. The fact that something exists simply means that it is perceived, but fortunately for the proverbial tree in the desolate forest, which is not perceived by humans or animals, Berkeley thought that it is nevertheless perceived by God's 
consciousness and thus exists, despite the fact that it is not observed by any mortal beings. Thus, according to Berkeley's metaphysical theory, everything is mental, ideal, conscious.

The Scottish enlightenment philosopher David Hume continued and radicalized Berkeley's idealism, among other things by pointing out that if Berkeley was right in that something exists only insofar as it is perceived by a consciousness, then the self does not exist because we do not perceive the self when we have conscious experiences. We can perceive the smell of coffee, the taste of liquorice and pain in our fingers, but to date no one has managed to perceive a self, which therefore according to Hume - must be considered non-existent. Hume was also inspired by Isaac Newton's mechanical physics, which had charted the laws of motion of physical bodies, and Hume believed that philosophy would chart the laws of psychological objects in a similar manner. In this sense, Hume is the grandfather of scientific psychology because he, as the subtitle of his impressive work $A$ Treatise of Human Nature indicates, was attempting to "introduce the experimental method of reasoning into moral subjects" (Hume, 1978). And by "moral subjects", he meant the phenomena which today are studied by psychology: how people think, feel and act, and not least what factors make them do so.

The German enlightenment philosopher Immanuel Kant (1724-1804) regarded Hume's conclusions as scandalous. But we can nevertheless place Kant's idealistic theory of the mind in the same group as Berkeley and Hume's "degenerate monism". Kant is undoubtedly one of the greatest philosophers and I cannot do justice to his views and arguments here, but the way in which his philosophy has been read by modern psychologists undoubtedly places him in the same camp as Descartes. He was read, for example, by the influential psychologist Howard Gardner, who, among other things, is the originator of the current theory of multiple intelligences, as an early cognitive psychologist who was interested in how knowledge is created when the outer world meets the various schemes, forms of intuition and conceptual categories that organize our experiences (Gardner, 1987). These schemes can be understood as an early version of what is known today as cognitive structures, i.e. the mind's way of structuring and processing information so that the world can be represented to consciousness and known. This is an updated version of Descartes' idealism, and whether or not Kant himself took this view (probably not) is not something I will attempt to resolve here, but in any case it is a fact that his philosophy has been used to legitimize certain types of mentalistic psychology in contemporary times. As previously stated, mentalism is the view that there are independently existing mental states and objects in an inner, mental world, which cause external behaviour. Regardless of their many differences, the common starting point for Descartes, Berkeley, Hume and (possibly) Kant is that the psychological realm is an inner world made up of conscious experiences.

As I mentioned before, very few researchers today, if any, accept Descartes' metaphysical dualism. However, there are many researchers who basically accept Descartes' way of formulating the problem, but who then deny the idealistic side of Descartes' philosophy and argue instead for the materialistic view that 
everything in existence consists fundamentally of physical matter. This kind of materialism is put forward by both behaviourists and physicalists. Both positions are reductionist. Behaviourism, in brief, is the theory that psychological properties are properties of behaviour, and physicalism is a theory that the mind is either equal to the brain in the strict sense (this view is called the materialist identity theory) or equal to the brain's functions (a theory that is called functionalism). Materialistic identity theory and functionalism are discussed in the next chapter, where I will try to shed light on the relationship between the brain and the mind (for there $i$ s undoubtedly a relationship, although it is not a relationship of sameness or equality).

The notion that the mind must fundamentally be understood as consciousness thus stems from Plato's dualistic teachings, via the Church Father Augustine and Descartes' establishment of modern philosophy and modern forms of thinking about consciousness, which accept Descartes' dualism between the inner and the outer, but which at the same time deny that inner conscious experiences belong to a non-material substance. This story ends, for now, with modern scientists, typically psychologically oriented neuroscientists, who work from Descartes' innerouter dualism and take it for granted that the mind is "inside the head" - more precisely, that it is in the brain as a materialistic version of Descartes" "thinking thing". One example of many is the trendsetting psychologist Steven Pinker, who says that the mind is what the brain does (Pinker, 1999).

\section{The mind as rationality}

The other main track in Western ideas about the mind is almost as old as the first and can be traced back to Plato's student, Aristotle. Aristotle was a great philosopher and scientist. He is responsible for having organized the sciences in a way that by and large has stood the test of time, and he was probably the greatest natural scientist in history until Charles Darwin (1809-1882). Plato's metaphysics had built on a distinction between eternal, immutable ideas, on the one hand, and the reflection of these ideas in earthly phenomena, on the other, and Plato ascribed the human soul to the realm of the eternal world of ideas, which is why the soul must be understood as being immortal. The naturalist Aristotle rejected this view: The soul - psukhe - is not immortal, since it cannot exist without the body, so when the body dies, the soul dies too. Aristotle's metaphysics more generally implied that form and substance, ideas and matter, always appeared together. There are no ideas in separation from their earthly incarnations; they are always immanent in the material world. Form cannot exist without substance, and substance cannot exist without form. Raphael's famous Renaissance fresco, the "School of Athens", illustrates the relationship between Plato and Aristotle on this point: Plato is seen pointing up towards the eternal heaven of ideas, while Aristotle points out in the world as sign that the ideal is inherent in things.

For Aristotle, substance is something that has the possibility of changing, while form is what makes a particular substance what it is. An object can change without 
changing form (for example, if you paint your car a different colour), but it can also change form in a substantial way (for example by melting the car's metal down and using it to make knives and forks instead). Forms designate the functions of things: the car's function is as a means of transport, while knives and forks function as eating utensils. According to Aristotle, everything has a function, and we can understand things fully only if we know their functions. Even if I were to map out all possible physical and chemical facts about a car (its dimensions and weight and its molecular and atomic structure), we can understand what a car is, strictly speaking, only when we know its function. The function - the purpose-of an object cannot be deduced from knowledge about the object's substance alone its physical and chemical nature, for example. Hundreds of years later, Martin Heidegger (1889-1976), as a modernized Aristotelian on this point, would make this a cornerstone of his existential phenomenology, according to which things of the world show up for us as Zeug or "equipment", defined by the ways in which they are "ready-to-hand" in patterns of practical engagement (Heidegger, 1927). A hammer, to invoke his most famous example, is first and foremost a tool that I can use to hammer, and is only in a secondary sense a detached object made from wood and iron (Heidegger thought that we become aware of the object as "presentat-hand" with such properties only when there is a breakdown in our practice, e.g. if the hammer malfunctions and we step back and begin inspecting it).

The fundamental difference between the metaphysics of Plato and Aristotle affected their views of the mind. I have already touched on how Aristotle regarded the mind or psyche as a biological concept. His book on the mind, De Anima, is not a modern psychology of mental phenomena in the broad sense, but rather a book on biological principles for life (Aristotle, 1957). One has to go to his works on ethics to find descriptions of human activities that resemble modern analyses in social and personality psychology (Aristotle, 1976). Aristotle understood the mind quite generally as "the animal principle of life", which in essence also applied to plant life. As a principle of life, the mind is the form of the living body, according to Aristotle. The mind is thus not a thing, an entity, but represents instead the defining properties of a living thing. Aristotle compares the relationship between a living organism and its mind to the relationship between the eye and vision. If an animal were an eye, the mind would be the ability to see. The mind is thus not an object that is "attached" to the body; it is not an object at all, just as vision is not an object attached to the eye. The human mind consists of abilities, capabilities and dispositions to know the world and act reasonably in it. Abilities and capabilities cannot logically be localized in physical space, which is why it makes no sense to try and find where the mind is localized (in the brain, for example). This is a grammatical point, as Wittgenstein would say.

As the living body's form, the soul is what makes the organism what it is. What makes a human being a human being is not only that we are alive, because beech trees and seagulls are alive too. What makes us human is the fact that our mind is rational. In addition to having motor and sensory functions, which our minds share with other animals, the human mind also has rational faculties. This 
is unique and defining for human beings. We are the only species that has a substantially complex language, and we are thus the only species that can reflect on our own actions and, for example, ask whether there are good reasons to act in a certain way. That is why we are also the only moral species in a strict sense.

Our rational faculties, according to Aristotle, can be divided into those that belong to theoretical rationality and those that belong to practical rationality. Theoretical rationality, according to Aristotle, refers to our ability to comprehend those aspects of reality, which exist independently from human doings and sufferings. Scientific discovery, such as in astronomy, is the paradigm for the application of theoretical rationality according to Aristotle. Practical rationality, however, is our ability to act rationally in our own lives, which is the domain of ethics, and in relation to the community that we are part of, which is the domain of politics. In this context, Aristotle uses the term phronesis, which is our ability to act prudently in the here and now while having an eye on life as a whole.

We train our practical rationality by being socialized into the collective world of social norms. As previously mentioned, it can be expressed with the phrase: "polis andra didaske" ("the city is man's teacher"). The rational mind comes into being in the organized community - which in Aristotle's time was the city-state. Our upbringing teaches us which events give us a reason for certain reactions, which is to say that we cultivate a sensitivity about when certain actions and feelings are legitimate or perhaps even required - and when they are illegitimate (McDowell, 1998). We do not learn merely to act mechanically, but to consider whether there are enough good reasons for us to do something. We thus acquire a "second nature". The mind has both a "first" and "second nature". The mind's "first nature" is our biological predispositions (e.g. instincts), of which we share many with the other animals, especially mammals and higher primates. The mind's "second nature" is then the acquired, encultured and socialized aspects of our lives - and not least our practical rationality. Theoretical and practical rationality are both essential abilities of the well-cultivated mind.

For the sake of completeness it can be added that, just as Augustine was crucial for the continuation of Plato's dualistic human understanding, the Catholic Church's most important philosopher, Thomas Aquinas (1225-1274), was crucial for Aristotle's thinking to be able to continue to influence Western medieval philosophy. Aquinas's major project was incorporating thinking about Christianity with Aristotle's philosophy. Aristotle's world view was fundamentally teleological, meaning that he believed that every object should be understood in the context of its purpose (telos in Greek). Aquinas endeavoured to combine this with Christianity's conception of a creative and sustaining God. But the Aristotelian teleological cosmology suffered a major blow with modern science's breakthroughs in the sixteenth and seventeenth centuries, when scientists like Galileo (1564-1642) and Newton (1642-1727) replaced the image of a purposeful universe with an image of a mechanical universe. The mind's place in this new scientific view was as a piece of machinery among other pieces of machinery. At least Hume was of this opinion, as we saw above, and it cleared the way for scientific investigations of 
the mind and later the establishment of modern scientific psychology in the nineteenth century, when psychologists began conducting experiments, for example, by confronting a subject with a particular stimulus in order to observe the causal responses. Because the teleological world view went under, Aristotle's approach to the mind did as well - at least until recently.

The breakthrough that resulted from this mechanical world view partly explains why there is no mention in the figure above of an Aristotelian, monistic understanding of the mind from the time of Thomas Aquinas in the thirteenth century to Wittgenstein in the twentieth century. This is illustrated by a dotted line from Aristotle to Wittgenstein in the figure above. Aristotle's, Aquinas's and Wittgenstein's ideas of the mind were incomprehensible within the mechanistic metaphysics that modern natural science was based on and helped to promote. How could there be reasons and purpose in a universe that could be comprehensively explained by causes and laws? How could there be meaningful actions in a world of purely physical events? These questions could not be answered within mechanistic metaphysics.

Part of Wittgenstein's philosophy consisted in breaking down the images that have kept us trapped intellectually since the mechanical world view emerged, including the image of the mind as some inner thing, and of the universe as a piece of machinery, so that we can better orient ourselves with regard to life's basic questions. We can say that Wittgenstein's basic understanding of the mind asserts that our mental life is not to be understood in a causal space, with causes and effects, but rather in a normative "space of reasons" (McDowell, 1998). We became acquainted with one example of an analysis of mental life in a normative space above when pointing out (with Smedslund) the conceptual relationship between experiencing surprise and an unexpected situation. This is a normative relationship, and not a causal or empirical one. This is because surprise is what you ought to feel in an unexpected situation, and if you do not, it is because you did not perceive the situation as unexpected. In much the same way, the relation that exists between bachelors and unmarried men is not causal or empirical. It is nonsense to say that being unmarried causes you to be a bachelor. Rather, it is a conceptual and normative relationship. Everyone who has mastered the term "bachelor" knows that they should (thus the normative) use it for unmarried men, since it is this class of people that the term refers to. The idea of the normative space of reasons plays a central role for me when we are to understand the human mind: the mental world is interconnected in a network of normative necessities, as well as in a network of causal necessities (Brinkmann, 2011a).

This also applied to Aristotle's thinking about mental life. When Aristotle discusses a psychological phenomenon like anger, he argues that this phenomenon cannot be comprehensively understood by scientists, who hunt for causalities or seek to reduce the phenomenon to its physical and chemical constituents (see Robinson, 1989). Natural scientists in Aristotle's day used to define anger as "boiling of the blood" (today we have other physiological and brain-related explanations) and this is not wrong, according to Aristotle, but it is only half of the story. To 
get the whole story, we have to supplement the scientific causal relation of the blood heating up with a normative or rhetorical explanation that considers the anger as a meaningful expression in an interpersonal situation. Today we would probably call the rhetorical side of anger discursive. Anger should be understood normatively or discursively as a response to having experienced injustice. Just as a bachelor is defined as an unmarried man, anger is defined as a justified response to experiencing injustice. We may well get angry in other situations too, but others will rightly reprimand us and say that there is no reason to be angry (just as we could use the term "bachelor" incorrectly and others will correct us). The physiological side of anger must be given a scientific, causal explanation, while the discursive side must be understood with reference to the specific situation that justified the anger normatively, e.g. if an injustice had actually occurred. Of course, it is a fact that people will react differently to a given situation - for example with anger - so an essential part of a psychological understanding is also about physiological differences among individuals as well as individual life story differences that manifest themselves in individuals within different discursive or normative frames of reference.

In somewhat technical terms we can say that anger as a psychological phenomenon has both a nomic side, i.e. it is part of nature's causal chains that can be described by laws, but that it also has a normative side, where the angry person not only can be described by laws, but can (if her mind is cultivated adequately) follow local norms for when anger is a legitimate response to events in the world. The order of non-human nature is purely nomic, while the order of human existence is also normative (Hacker, 2007). Nature cannot be said to follow natural laws and therefore it cannot break them - rather, it can be correctly described by these laws. People can, however, follow social norms and are therefore also capable of breaking them. A list of opposites is summarized in Table 1.1.

In this book, I argue that the normative side logically prevails over the nomic side when it comes to psychological phenomena. If a phenomenon existed solely in a nomic and not a normative context, it would be wrong to regard it as a psychological phenomenon and then potentially study its physiological correlate. We investigate the physiological correlate of something only when we know what it correlates with (e.g. anger). The reason that, for example, we perceive anger as a psychological (normative) phenomenon, but fatigue or constipation as purely physiological (nomic) phenomena, then, is that only anger exists in what can be

Table I.I The nomic and the normative

\begin{tabular}{ll}
\hline Nomic & Normative \\
\hline Cause & Reason \\
Behaviour & Action \\
Behaviour can be described by the & Individuals can either follow or break \\
laws of nature and statistics & rules and norms \\
\hline
\end{tabular}


called a moral order where it makes sense to look for a reason to be angry (Harré, 1983, p. 36). Anger exists in webs of normative necessities in human social practices, since it can be justified or unjustified, but this does not apply to fatigue or constipation. We can have a reason to be angry, whereas fatigue and constipation are not explained with reference to reasons, but to causes instead. There are situations in which you should be angry, when you experience a blatant injustice that violates an individual's rights, for example, and there are situations in which anger is inappropriate, for example, getting angry with an infant who is not yet responsible for its actions. But we cannot say in a corresponding manner, that there are situations where you should or should not be constipated. Constipation is not a normative phenomenon - and is therefore not psychological (although it may well be related to psychological processes, say, if it turns out that angry persons are in higher risk of suffering from it).

I will continually return to the normative view of the mind in the following pages. This view applies not only to emotions like anger, but equally well to reasoning, memories, actions, perceptions, and other psychological phenomena. When we argue, for example, our reasoning is open to judgement in the context of the normative standards of good reasoning; the most basic of these standards come from logic. Reasoning is thus a psychological and not, for example, a physiological phenomenon. When we remember, we try to remember correctly, which means that remembering is a normative phenomenon. And when we act, the action can be judged from a variety of different norms (e.g. ethical, aesthetic, instrumental), but the fact that something can be judged is what makes us call it an action rather than a mere physical movement. If a doctor tests my reflexes by tapping below my kneecap, my reaction is not to be judged as a more or less correct action (in the normative sense), nor am I to be morally blamed for my reaction. The reaction is instead regarded as purely mechanical: Does the reflex mechanism work or not? The reaction is in fact not an action, but is pure behaviour, pure movement. Thus we are dealing with a nomic process here. But if I move my leg in a way that is physically identical to the reflex movement, but this time in order to kick a ball and score a goal in a football match, then we are dealing with another type of process; i.e. an action that can be judged as more or less good and appropriate in relation to the norms that define the game of football. Here we are back in the normative world, hence in the psychological domain.

In the tradition of Aristotle and Wittgenstein, the mind is, in short, something normative. The mind is not a "thing" that can be comprehensively understood as an element in the chain of mechanical causes and effects in the physical world, nor is it a "consciousness principle", as Descartes believed. The mind is "a distinctive range of capacities of intellect and will, in particular the conceptual capacities of a language-user which make self-awareness and self-reflection possible". (Bennett \& Hacker, 2003, p. 105). In other words, the mind is an array of rational faculties, a widely ubiquitous term for our abilities and dispositions (Coulter, 1979, p. 13). The mind is not a term that indicates a location or a thing: "the mind is not a thing; talk of our minds is talk of world-involving capabilities that we have and activities that 
we engage in" (Putnam, 1999, pp. 169-170). As psychologists, we should therefore develop an understanding of the mind that takes into account the fact that there is a much closer relationship between the mind and the world than Descartes' dualisms have led us to believe. From the viewpoint of "world-involving capabilities" (to use Putnam's apt phrase) there is no more a metaphysical divide between the mind and the world than there is between our hands and the things we manipulate. The mind should be understood as a complex extension of our hands, which allows us to do something in the physical world - which therefore can be assessed normatively (Brinkmann \& Tanggaard, 2010). The pragmatist philosopher John Dewey expressed this thought by saying that we should not perceive the mind as a noun but as a verb:

Mind is primarily a verb. It denotes all the ways in which we deal consciously and expressly with the situations in which we find ourselves. Unfortunately, an influential manner of thinking has changed modes of action into an underlying substance that performs the activities in question. It has treated mind as an independent entity, which attends, purposes, cares, and remembers.

(Dewey, 1934, p. 268)

In reality, the mind is not a thing behind our actions that does something, but is instead a term for the abilities we have to do anything in the first place, which can then be judged as more or less correct, adequate, good or productive (for Dewey's views on the mind, which, like Wittgenstein's, are essential for this book, see Brinkmann, 2013).

I have presented this other track in the history of Western thought about the mind as a rationality point of view in order to emphasize this perspective's focus on the significance of normativity for psychological phenomena. That is to say that the normative and the rational are related. Something is a psychological phenomenon within a normative framework only if one can distinguish between it being more or less correct; for example, more or less correct actions, perceptions, memories or emotional reactions. The fact that this point of view is called the rationality viewpoint does not mean that irrational things (e.g. phobias) are not a matter for psychology. Phobias are psychological (and not physiological) problems, precisely because we judge them in the light of standards of rationality, which is something we do not with, say, appendicitis (which is therefore not a psychological problem, but a physiological one). Moreover, this viewpoint does not entail excluding feelings and emotions from the field of psychology or having psychology deal only with thinking and cognition. On the contrary, it is about the fact that feelings are psychological to the extent they can be evaluated to a greater or lesser degree based on reasons - in other words, that they are more or less rational. It is rational to fear fearsome things, because a sense of fear will encourage us to escape, but it is dysfunctional to be afraid of harmless things. If something is completely outside the sphere of the normative - outside of the normative space of reasons, where it makes sense to ask people for rational explanations for their 
thoughts, actions and feelings - then the phenomenon, like in the case of constipation, belongs to physiology (or similar sciences), but not to psychology. ${ }^{2}$

One should, therefore, not misunderstand the rationality viewpoint as expressing an over-intellectualization of our mental life. The vast majority of everything we do takes place habitually and unconsciously and it is only when things go wrong or when we run into problems that we need to stop and use our reason and our ability to reflect (Dewey, 1922). The point is thus not that everything we do is rational. The point is rather that everything we do - if it can reasonably be regarded as psychological - must potentially be able to be considered relative to normative frameworks. So: the fact that I cry when I see a sad film is a spontaneous and immediate process, but it is psychological, because I can later consider whether I might have misunderstood the film and overlooked the fact that it was not sad at all (but comedic instead, for example). The next time I see the film I may have realized that there was not any reason to cry at all, but a reason to laugh instead. I cannot reflect in a similar manner on my digestive system's reasons for reacting with constipation when it deals with a certain type of food, because it has no reason to react like it does. It merely reacts as a purely physiological and chemical process, which is precisely why digestion is not psychological and is instead a phenomenon rightfully studied by physiologists. The rationality viewpoint will therefore in no way deny that we are often irrational, driven by emotions, or immoral. Something can be immoral or irrational only if it can also be moral or rational. The rationality viewpoint insists that when we talk about psychological processes, we talk not only about something that happens, but about something that is done - and therefore we can always judge, at least as a matter of principle, whether it is done well or poorly, morally or immorally, rationally or irrationally.

\section{Mindism and personism}

An immediate question arising for the rationality viewpoint, however, is about the reference of psychological terms. If the concept of mind names our rational powers and dispositions, then who has these powers and dispositions? It is not the mind that has them, because that would be circular. A key point of the present book is that it is wrong to ascribe psychological predicates to the brain, but it is equally wrong to ascribe them to the mind. The brain does not think, but neither does the mind (Bennett \& Hacker, 2003, p. 105). It is misguided to say that "my mind is sad" or "my mind remembers what I had for lunch yesterday". It is correct to say that $I$ am sad, or that $I$ remember something. This $I$, however, does not refer to an inner experiencing or thinking thing, because it does not refer at all. Rather, saying "I am sad", for example, is a mode of expressing sadness for linguistic creatures. As Wittgenstein would say, "I am in pain" does not refer to anything, but is an expression of the pain one feels. In short, it is only the person who can be sad and remember yesterday's meals. It is not the person's brain (although the brain is needed for the person to have emotions and memories), and it is also not the person's mind. The mind stands for the person's abilities to feel emotions, remember 
things past, and much else, but it is irreducibly the person who does these things, and not the mind. Similarly, we can say that the car moves through the city. Moving is here equivalent to the mental functions of a person, and we cannot sensibly say that the car's movement is what is moving it (just as we cannot sensibly say that the person's mind is doing the thinking).

This means that we need a robust concept of the person as fundamental in psychology. A forceful argument to this effect has been advanced by Elmer Sprague (1999). He invokes a useful distinction between two philosophies of the mind, which he calls "mindism" and "personism" respectively. Mindism is very close to what I have described as the consciousness track in Western thought about the mind. Sprague mentions Descartes, Locke, Hume, Davidson, Fodor, Dennett and Searle, and he could include many other contemporary cognitive scientists. Mindists take what Sprague calls a "spectator stance" to mental phenomena and ascribe psychological properties to the mind as a peculiar kind of thing. Personists deny that the mind is a thing or an object, and they make the person the only subject of mental verbs (p. ix). Representatives of Sprague's personism are much rarer than mindists in the history of philosophy, and he focuses on just three: Aristotle, Wittgenstein and Ryle (1949). The personists attack the spectator stance of the mindists, according to which we "observe" each other - including the "contents" of our own minds - as if they were objects. Instead, personists defend an agent stance, according to which acting persons in relationships represent the prime psychological reality. Psychology should thus primarily be about persons and their doings, and minds do not exist as special kinds of objects. There is no "intelligence" as a reified object, for example, locked in a mind or brain, but only people acting (more or less) intelligently. There is no inner "memory" in a reified sense, but only people remembering things (more or less) correctly. This may seem like behaviourism, but it is not, for it does not involve a denial of the obvious fact that persons experience many things that they do not have to share with others, and, also unlike behaviourism, the notions of meaning, reasons and actions become central to understand the lives of persons - rather than mechanism, causes and behaviours. This whole book can be read as an attempt to develop a "personist" foundation for scientific psychology, and I return later to the central concept of the person.

\section{Science of the mind: A brief scientific history}

In addition to this book's perspective on the mind as a range of rational abilities and dispositions that can be ascribed to living persons, we have a wide range of sciences that study the mind. Not only psychology but also sociology and social anthropology deal with the human mind from a social and cultural perspective, respectively. But psychology naturally has a certain centrality as the principal science of the mind, especially today, when we live in a psychological society, because we all use psychological concepts to understand our own and others' behaviour - shyness has become social phobia, sadness has become depression and personal imbalances are now often seen as personality disorders, to name just 
a few psychological difficulties (for a critique of diagnostic pathologization of human life, see Brinkmann, 2016a).

Consistent with the two-sided image of the mind that I have outlined as having both a nomic and a normative side, scientific psychology has also been two-sided throughout its historic development. This has given rise to great battles within the science over the correct way to regard the mind. This helps to explain why psychology is full of dualisms like mind-brain, individual-society, action-behaviour, causereason, explanation-understanding, quantitative-qualitative and many others. It seems that when we talk about human life in the psychological sense - in other words, not merely about purely biological life but about individuals' existence then we inevitably run into such dualisms. Some researchers have argued this means that we should have two psychologies: the first psychology would be experimental, quantitative and explain by causes, and the other psychology would be descriptive, qualitative and understanding by interpretation (the locus classicus is here Dilthey, 1977, as we shall see below). This distinction corresponds roughly to the distinction between the nomic and the normative.

The relationship between these two psychologies is a thread throughout most of the history of psychology, and unfortunately modern psychologists have often been far less nuanced than Aristotle, who, as mentioned, respected the hybrid nature of psychological phenomena. Modern psychology is very much a product of the British empiricism of the 1700s and especially of David Hume's attempt to develop a science of the mind using Newton's mechanical physics as a model. Hume wanted to apply a way of thinking to the mind that was experimental and explained by causes, just as Newton had successfully done with the physical world. Hume never really realized this program, but the later empiricist John Stuart Mill (1806-1873) did; Mill has been singled out as being primarily responsible for having created modern scientific psychology since he formulated its scientific theoretical basis (Gazzaniga \& Heatherton, 2003).

Mill insisted on the duality between human and social sciences. In addition to an elementary psychology that explained causes on the basis of experiments, Mill thought there was a need for what he called "ethology", which was to be the science of the formation of the human character. Mill's ethology has nothing to do with modern biology (today the term ethology denotes the study of animal behaviour), but refers instead to the Greek concept of character and custom: ethos. Ethology is the study of human character and morality. Mill's viewpoint was that human character is formed under very specific circumstances - historical, social and cultural and this is why it is difficult to lay down universal laws for it. At one point Mill says that only "an oriental despot" would be able to educate people under the strictly controlled conditions necessary in ethological experimental science, and even then it would be impossible to control every single character-forming impression (see Cahan \& White, 1992, p. 226). In Mill's major work, A System of Logic, published in 1843, he classifies psychology among "the moral sciences" and he believes not only that psychology's subjects belong to the moral (i.e. the normative) domain in a broad sense, but also that psychology's mission is to contribute to humanity's 
improvement in accordance with utilitarian principles (Mill, 1843). In any event, Mill was operating with a dual psychology: an experimental one and an "ethological" one. Both of them, however, should be inductive and empirical. When Mills' work was translated into German in 1849, "moral sciences" became "Geisteswissenschaften", i.e. sciences of the spirit (Kessen \& Cahan, 1986). "Geisteswissenschaften" was a new word in German at the time, at least in the plural form, and it paved the way for Wilhelm Dilthey (1833-1911), who, if anyone, stands out as the one to bisect the science of psychology, as we shall see.

Dilthey is famous for having made clear the methodological differences between the natural sciences, on the one hand, and the humanities and social sciences, on the other (Dilthey, 1977). His most famous quote is: We explain nature; we understand mental (or spiritual) life [Die Natur erklären wir, das Seelenleben verstehen wir]. This means that the nomic, causal world must be explained by uncovering the laws of nature, while the normative world must be understood by grasping the norms that order our historical lives. Mental (spiritual) life is, above all, historically determined, according to Dilthey, which is why we do not get a real insight into it through introspection, i.e. by turning "inward" into our own psychological world, or through experiments, but only by considering how it is historically embedded. If there were to be a science of humans and their minds, claimed Dilthey, then it should be an anthropology that aims to understand the totality of human experience in its context. It is commonly recognized in historical philosophy that we cannot formulate general historical laws like "every time an heir to the Austro-Hungarian throne is shot in Sarajevo, a new World War breaks out" - precisely because historical events are unique.

If natural science, in its essence, works deductively-nomologically, that is to say that it explains an individual phenomenon by subsuming it under a general law, then we must conclude that man's historical and mental life does not lend itself to scientific study. As far as psychology is concerned, the conclusion is therefore that if its subject is historical, as Dilthey believed, then psychology should not belong primarily to the natural sciences. The theoretical scientific discussions that Dilthey set in motion have been very significant for later cultural thinkers in psychology - not least for hermeneutically oriented psychology, such as some of the cultural psychology discussed in Chapter 4, represented by names like Richard Shweder (1990a) and Jaan Valsiner (2014).

These discussions were also significant from a purely methodological perspective since they formed two basic ways to investigate the psychological realm: on the one hand, we can use various types of experiments in which researchers seek to isolate a specific factor that they believe will be able to explain a particular phenomenon. For example, if we are interested in what conditions are necessary for people to behave generously, we can create a psychological experiment. We then need an experimental group in which people are asked to donate money, for example, while they know that they are being observed, and a control group in which people believe they are not being watched. And we will then possibly find that statistically there is a significant difference and that people are more generous 
when others observe their behaviour. We then seek to explain the generous behaviour as being caused by this particular factor. The behaviour is thus approached as something that simply happens.

While this experimental logic regards humans as natural objects that, like everything else in the universe, work on the principle of cause and effect, other methodological traditions take a completely different approach. Within the field of what is called qualitative research, there is insistence on understanding people's actions as meaningful, i.e. people are viewed as meaning-making agents and, for example, selected individuals are interviewed about their experiences of generosity and this is potentially supplemented with field studies of human activities in real situations (Brinkmann \& Kvale, 2015). Reducing the complexity of the human world through an experiment is not the goal in this tradition; it is rather to study the psychological processes as wholes in the real and complex contexts where they actually play out. One seeks to understand and not to explain. Generosity in this context is not something that just happens, but something that is done in the form of generous actions that people can talk about.

Here we have the basic distinction between the quantitative and qualitative methods, and this is reflected in psychology's method books and has given rise to many fruitless battles. In reality the methods are not in competition since they do not provide different answers to the same question. On the contrary, they provide different answers to different questions. When you conduct experimental research with quantitative methods, the question you ask is "how much". For example: "How much of human generosity can be explained by the fact that we want to be seen positively in the eyes of others?" Within qualitative research, however, we ask more descriptive "what" and "how" questions, such as: "What is a generous act from people's perspectives?" or "How do we experience generosity?" Qualitative methods have been marginalized for decades and have often been accused of being subjective and unscientific because they do not give results in the form of a numeric value. Just as this book will show that normative psychology is inevitable, it will also show that this applies to qualitative psychology as well: We must logically know what the psychological phenomena are qualitatively, and describe how they are played out in social and cultural practices, before it makes sense to ask "how much" questions that seek explanations.

A large number of psychologists since Dilthey have dealt with the dualization of psychology - in Europe, USA and Russia. One from each of these geographical regions can be mentioned here: Wilhelm Wundt, John Dewey and Lev Vygotsky. Wilhelm Wundt (1832-1920) is the official founder of psychology, according to scientific mythology, since he created the first psychological laboratory for the study of psycho-physical processes at the University of Leipzig in 1879. But beyond this experimental part of psychology, he also created the other side of the science of the mind, which was based on ethnographic data about languages, myths and culture: a Völkerpsychologie. In the American context, the first psychology textbook was written by John Dewey in 1887. In a later article from 1917 entitled "The Need for 
Social Psychology", Dewey says that all psychological phenomena can be divided into either the physiological or the social, and when we have relegated elementary sensation and appetite to the former, all that is left of our mental life, our beliefs, ideas and desires, falls within the scope of social psychology (Dewey, 1917, p. 54). A modern successor of Dewey's thinking, the late Richard Rorty, believed that, by extension, mentalistic psychology will go under as developments are made in brain research, in much the same way that alchemy did after chemistry emerged as a mature science. For Rorty, there was no mental level between the brain and culture. Therefore, it is not the mind that is the brain's software, as modern psychologists have otherwise asserted. According to Rorty, culture - social life - is the software: he said that "culture is to software as the brain is to hardware". (Rorty, 2004, p. 231). We see here that Rorty dissolves psychology into its neighbouring sciences, which seems to be a constant danger for psychology because of its duality or hybrid nature. On the one hand, the fact that psychology has such a close relationship with both the biological and sociological sciences has provided a rich science, but, on the other hand, it has meant that it has been regularly argued that psychology is therefore superfluous. The argument in this book is that if psychology is constructed on the basis of either mentalism (mindism) or reductionism (which we may call "brainism", paraphrasing Sprague's distinction), then Rorty and others are correct in saying that it is without justification. But if we start from a non-mentalistic and non-reductionist psychology (personism), which does not regard the mind as a "mental thing" but as a range of rational abilities and dispositions of living and acting persons, then psychology has an important place among the sciences as the study of how people act and create meaning.

One of the non-American psychologists who was engaged in this kind of discussion was the Russian Vygotsky, with whom we will become acquainted later in Chapter 4. Vygotsky wrote insightfully about the crisis in psychology in the late 1920s (Vygotsky, 1927). The crisis consisted in scientific fragmentation and dualisms. Vygotsky believed that the two psychologies should be supplemented with a third psychology - primarily on Marxist foundations. The crisis in psychology was precisely about the fact that there existed many different psychologies, which, however, were assembled in two general families: an explanatory psychology belonging to the natural sciences, on the one hand, and a descriptive, phenomenological psychology, on the other (phenomenology will be explained in Chapter 3). Vygotsky viewed the diversity in psychology as a sign of scientific immaturity, but dualism is nevertheless - and productively so - clearly reflected in his important work to develop a cultural and historical psychology of the "higher mental functions". He perceived these as being created by linguistic signs and symbols and (other) tools, and as being something other than the mental functions that are biologically determined. For Vygotsky, there is thus a clear distinction between lower and higher, biologically determined and culturally mediated, which is not far from the nomic and the normative dimensions discussed above. 


\section{Conclusions}

Throughout this book I will touch on many kinds of psychological research that come from both "the first psychology" and "the second psychology". A goal of the book is to show that the two psychologies can and must coexist, but that we must take care to elucidate the proper relationships between them. This will be addressed directly in Chapter 6. First and foremost, we need to maintain that the "second psychology", the descriptive, understanding, interpretive and normative kind, is justified and necessary for a proper understanding of the mind, even though mentalism and reductionism deny this, each in its own way. In this chapter I may not have delivered any real "knock-down argument" against the mentalistic and reductionist traditions that stem from thinking about the mind as consciousness (my first track in the history of ideas), but I hope that it will become clear in the following chapters why it is a much better alternative to think about the mind as rationality (my second track in the history of ideas from Aristotle to Wittgenstein) and in terms of personism. In summary, we can say that the first track considers the mind to be a mechanism that primarily represents the world and thus makes us conscious of properties of the world, while the second track considers the mind to be a name for the rational abilities and dispositions of persons to act in the world. Whereas the first track is concerned with uncovering the causal laws that are believed to determine how we represent the world in our minds, the second track is concerned with our ways of acting as always being open to normative judgements and interpretations. The psychological realm in the former case is thus something mechanical and causal, whereas in the second case it is meaningful and normative.

In my opinion, there are such major problems in regarding the mind as a representational mechanism, regardless of whether the representations are Cartesian ideas or cognitive psychological information bits, that the first main track creates far more scientific and philosophical problems than it solves: What is it about a mental representation that makes it represent what we think it does? If mental representations exist, where are they and what form do they take? How can one person communicate her mental representation to another person? How do we even know whether our mental representations correspond to anything in the outside world? As we shall see in the following chapters, once we ask these questions we run into a philosophical impasse. The cure is not to provide better answers to the questions, but to start from a completely different tack; and that is to ask the questions that the Aristotelian tradition would ask. Here, the fundamental question is not about how the mind (the inner world) can represent the outer world. Instead, a fundamental question is: If the mind is a range of abilities and dispositions, what do we need to know in order to be able to understand how these abilities result in actions? My proposal for an answer will be given in the following four chapters on the brain, the body, society and things. These are regarded as layers of mediators that make possible the sophisticated psychological life forms of persons. 


\section{Notes}

1 Not all philosophical or psychological traditions envision consciousness as "an inner world". For example, the phenomenology of Edmund Husserl (1859-1936) is concerned with describing consciousness or the mind as "intentionality", i.e. as a directedness that always points beyond itself and refers to something other than itself, which was the inspiration for phenomenological psychology (e.g. Giorgi \& Giorgi, 2003). This is closely aligned with the perspective of the present book and its focus on rationality and more precisely normativity. There was in Husserl's phenomenology an acute awareness of the normativity of our experience. Intentionality was certainly a key concept from him, which he took from Brentano. It means that experience is always about something our thoughts, feelings, perceptions and actions are always directed at something. But, as Crowell puts it, "intentionality is not simply the static presence of a "presentation' in a mental experience (Erlebnis) but a normatively oriented claim to validity." (Crowell, 2009 , p. 13). In colloquial terms, this means that what we experience can only be "about" something (intentionality), because there are more and less correct and valid ways of experiencing it (normativity). For example, we may see a dangerous snake in the forest, but - on closer scrutiny - it may turn out to be an innocent branch, and our intentional orientation toward the object involves a normative underpinning of trying to "get it right". Simply put, it means that we experience normativity, valences and values in objects and events - something the Gestalt psychologists took up after Husserl (Köhler, 1959).

2 I am well aware that I am conveying a minority view within scientific psychology here, and the reader should of course be aware of this as well. The vast majority of modern psychology can be understood as seeking to uncover causal relationships rather than to elucidate normative connections in the social world. The forms of cultural psychology and discursive psychology conveyed in this book, and which, like me, put forward the view that normative explanations are far more important than causal explanations in psychology, represent a small portion of psychology overall; the vast majority is still positivistic (see Harré \& Tissaw, 2005, pp. 16-19). 


\section{The mind and the brain}

Today, many people - scientists and laymen alike - will as a matter of course say that the psychological realm is essentially an aspect of the brain. Many people have a notion that the mind is in the brain or even that it is the brain. The mind is what the brain does, as it is sometimes expressed. The psychological realm is perceived as something intracranial, something "inside the head". This notion is also prominent in the public debate in my own country, Denmark, for example, where Lone Frank, a neurobiologist and science journalist, with a best-selling book on The Fifth Revolution (Den femte revolution) - the revolution of the neurosciences after the scientific revolutions of Copernicus, Darwin, Freud, and Watson and Crick - has made a significant contribution to the public discussion about the possibilities and implications of brain science in terms of human self-understanding. The book makes it clear that, at present, brain explanations are increasingly replacing psychological, humanistic and social-scientific explanations for human behaviour: "Whereas in the past speculations were made with reference to culture and to a mind that was curiously detached from the organism, the physical brain is now steadily becoming the reservoir and terminus for all of the questions we put to human nature and existence." (Frank, 2007, p. 18; my translation). As a reader of newspapers in both Denmark and the English-speaking world, it is not difficult to recognize this description all too well: the results of neuroscientific research take up more and more space, often coupled with an interest in evolutionary psychology. Biological and genetic explanations of human behaviour are increasing, while culture-oriented explanations are on the decline.

According to Frank, this is a sound consequence of advances in brain research; it is a consequence of the fact that we are coming to realize that we are our brains: "my brain - it is me!" (Frank, 2007, p. 17). When we acknowledge that we are our brains, then it is of course in the brain we need to look to learn more about ourselves. The way to get to know ourselves is not through individual self-discovery as in psychotherapy, for example, nor is it through a preoccupation with the historical and cultural conditions in which we live our lives. When we are our brains, it is obviously the brain that is interesting and not our biography, culture or history. And, according to Frank, we also need to accept that we are "responsible for own brains" (p. 140). Responsibility for dealing with life's essential tasks can 
no longer be "pushed over onto a convenient collective and shapeless mass like 'society'. The individual is irretrievably on the path back to the centre" (p. 114). The individual is back because the brain is back - and your brain is precisely your brain; you are your brain. Frank is very good at formulating sharp ideas, but they are nonetheless representative of a pervasive and significant way of thinking about humanity today.

When, according to such current ideas, we are our brains, it is clear that brain sciences obtain a centrality that more traditional humanistic perspectives on man have had in the past. It is also understandable why brain research has been expanding into various other subjects in recent years, resulting in peculiar configurations like neuro-ethics and neuro-theology, and also why brain research has entered into a partnership with the commercial sector, for example. According to newspaper stories, the neuro-marketing firm Mindmetic, for example, has developed an emotion detector that uses brain measurements to enable advertising messages to be best adapted to consumers and their emotional preferences. ${ }^{1}$ The company Nordic Living will be "emotion-testing" 100-125 new products in this way, and it can predict how an advertisement will be received with $75 \%$ accuracy. It could be argued that it would be cheaper, easier and more reliable to simply ask the consumers who are exposed to the advertisements. But the people behind neuromarketing conclude quite the opposite: companies will be able to save money by using brain methods because traditional qualitative methods like focus groups are too slow and imprecise. The chairman of the Consumer Council in Denmark has understandably sounded the alarm and fears that, in the future, neuro-marketing will hit the mark every time so that our patterns of consumption will be able to be controlled remotely.

My view is that this debate is interesting primarily because of the surprisingly deep faith in brain research that has been flourishing in recent years. This faith suggests that we view neuroscientists to a great extent as the new wise men or priests who possess unquestionable knowledge about man that is far more tenable than that of other professions. If you take a look behind the catchy rhetoric that is used in neuro-marketing, for example, there is not much evidence to suggest that brain research could ever become a "remote control" for people - in much the same way as with the arguments and conclusions that Lone Frank so enthusiastically puts forward in her book. There are a lot of exaggerated, science-fiction conclusions and hot air in the debate about the brain and the mind. In this chapter, I will show that it is simply philosophically and scientifically untenable to say that "we are our brains" (Noë, 2009, likewise puts forth a number of such arguments). I will also argue that this notion of the brain has questionable ideological effects on our society. It will simply impoverish our lives if we "voluntarily" become part of the project, which in recent years is in the process of reducing thinking, feeling and acting people to their brains. If we start using these ways of thinking and talking about the brain, which are quite legitimate and indispensable in neuroscience, to understand our mental life more generally, then we will cut ourselves off from understanding key aspects of ourselves. 


\section{The brain as an organ}

There is no doubt that the brain is an amazing organ that we have actually come to know a great deal about. In humans the brain is the part of the central nervous system that is located inside the skull. The spinal cord is also part of the central nervous system, and it runs from the brain down to the second lumbar vertebra. All vertebrates have brains, but other living beings manage without. Jellyfish, for example, have decentralized nervous systems that are not controlled by a central organ, and mushrooms do not have a nervous system at all. The brain of an adult human weighs about 1,400 grams; a little less in women, and a little more in men. It contains about 100 billion neurons, which are nerve cells, each of which communicates with approximately 10,000 other neurons (Bloom \& Lazerson, 1996). This makes the brain an incredibly complex organ. The number of possible neural circuits is hyper-astronomical - the number has to be written as 10 followed by at least a million zeros. In comparison, the number of particles in the universe is written as 10 followed by "only" 79 zeros (Edelman \& Tononi, 2000, p. 38).

The connections between the neurons of the brain are made electrically and chemically via synapses, which are the spaces between the neurons where neurotransmitters, or chemical messengers, transmit impulses from one neuron to the next - or from one neuron to other cells such as muscle cells. The neurons emit chemical messengers that cross what is called the synaptic cleft and bind to specific receptors on the postsynaptic neuron. The whole process takes place in a matter of milliseconds. When a neurotransmitter binds to the postsynaptic neuron, it becomes less negatively charged, and if it becomes sufficiently negative, then it "fires", meaning that it conveys the impulse to the other neurons to which it is connected (Edelman \& Tononi, 2000, pp. 40-41). Some of the most important systems of neurotransmitters are amino acids and monoamines, which include norepinephrine, dopamine and serotonin. Changes in these last three may be related to psychological changes, where, for example, a well-known (but highly controversial and quite possibly wrong) hypothesis is that depression is related to serotonin deficiency. The well-known SSRI antidepressants work by inhibiting the reuptake of serotonin, thereby increasing the concentration of the substance in the brain.

The brain consists of different structures, which can generally be divided into the cerebrum and cerebellum. The cerebrum is involved in higher mental functions such as memory, thinking, problem solving and the ability to speak, while the cerebellum is involved in the control of more automatic functions, including, for example, the ability to keep one's balance. Respiration and similar basic processes are controlled by the medulla oblongata, which connects the brain to the spinal cord. Some specific areas of the brain can be designated as being more or less directly associated with a specific function; for example, Broca's and Wernicke's areas in the left hemisphere are active in language and speech, but many other mental functions are not similarly associated with a particular, isolable area. Often - and possibly always - the cerebrum as a whole is involved as the neural basis of a mental function. 
The question of cerebral localization, i.e. whether the cerebral foundations of specific mental functions can be localized to specific areas of the brain, has been heavily debated throughout the history of brain science and continues to be controversial today (Gerlach \& Starrfelt, 2007). An early view from the eighteenth century was formulated by what is called phrenology, where the leading figure of the movement, Franz Joseph Gall (1758-1828), believed that personality traits and abilities were represented in specific parts of the brain. He also believed that it was possible to determine how well developed these traits and abilities were in individuals by measuring the size of certain locations on their skulls. If your skull bulged a lot on the morality spot, then you were a highly moral person.

Phrenology is often ridiculed today, but we would do well to remember that in its time it was a highly respected scientific approach with its own scientific centres, journals and standards for good phrenological research. Nowadays neuroscientists do not measure the size of bulges on the skull when conducting neuroscientific studies. Instead, they can directly measure activity (e.g. blood flow) in different parts of the brain using various imaging techniques and create a graphical representation of this activity, like the ones that most people have seen pictures of in books or on television. Regardless of how amazing these imaging techniques are from a purely technological standpoint, it is still unclear whether they are as conclusive in the scientific sense as some neuroscientists believe. First, many such studies are built on a principle of high cerebral localization, which is debatable, and second, it is uncertain whether the activities that can be measured in the brain are crucial for the mental function that one is interested in. It is possible that the activity in a particular place in the brain is merely a concomitant of a function rather than playing a crucial role in the function (Gerlach \& Starrfelt, 2007 , p. 446). When some neuroscientists today claim to have found the place in the brain where morality or love is located, then it is, in my opinion, just as wrong as when phrenologists believed they could measure a person's morality or love based on the bumps on their skull. I think that history's verdict on the simplistic conclusions in current brain research will be just as tough as today's verdict is on phrenology.

As many will know, the brain consists of two halves, and a lot of pop science and New-Age thinking has adopted the view that the left brain is analytical and intellectual, while the right brain is holistic and creative. This should probably be considered as both conceptual nonsense (because a hemisphere cannot be "creative" in any meaningful sense) and also scientifically wrong, because in reality both hemispheres are involved in the vast majority of mental functions. Nevertheless, it is an example of how brain research influences people's thoughts in their everyday lives. Sometimes this notion of the two hemispheres is coupled with the idea that men and women use the one or the other half, respectively, the most. And at other times this idea of brain differences between the genders is used to legitimize different teaching methods for boys and girls in primary schools. In Denmark, the teacher Ann-Elisabeth Knudsen has been an advocate of this view in the public debate, which many school leaders have embraced, and she believes 
that boys become losers these days because they are subjected to an educational approach that is best suited for girls' brains. On her website, she writes that boys should be forced to do at least a half-hour workout with bead plates every day, as she believes this will strengthen the linguistic area in their frontal lobes.

Based on such more or less well-founded recommendations, it is good to remember what it is that the brain actually does when it shapes and determines our body's functions - biological as well as psychological. And the brain's function, as an organ, is basically to send electro-chemical signals along specific circuits. This happens as a causal or nomic process, as I called it in the previous chapter. These electro-chemical processes are necessary for our organisms to be able to live, breathe, keep balance, move and so on. Our biological functions are thus dependent on cerebral processes, and our mental functions are as well. Without brain processes we could not think, feel or act. That is a simple truism.

But does that mean that mental functions are in the brain? Does it mean that thoughts and feelings are "inside the head"? Does it mean that it is the brain that thinks, feels and acts? I have an adverse opinion on the matter. Although having a brain that functions in a certain way is a necessary condition for the existence of mental life as we know it, it is not the same as saying that mental life is equal to the brain's functioning. As previously mentioned, it is similar to a vehicle: you cannot drive a car without an engine, but that does not mean that it is the engine that is driving. It is the car as a whole that is driving, just as it is the individual who thinks, feels and acts - and not that individual's brain. My argument is not empirical here, and no empirical data can be found that speaks either for or against it. Instead, it is an argument based on an analysis of the meanings of concepts. Regardless of what empirical neuroscience might teach us, it is a mistake to attribute abilities and dispositions to the brain, which make sense only when attributed to the organism as a whole.

Many people - scientists and laymen alike - make this mistake, which has been dubbed "the mereological fallacy" (Bennett \& Hacker, 2003). Mereology is the study of the relationships between parts and wholes, and the mereological fallacy consists in attributing characteristics to a part of something (e.g. the brain), where it is meaningfully attributable only to the whole (e.g. the organism). The worldfamous neuroscientist Antonio Damasio, for example, commits a mereological fallacy when he describes how the brain is aware of an image of an object, or when he analyzes how the brain perceives something (Damasio, 1994, p. 263; 1999, p. 189) (see also the extended critique of Damasio in Brinkmann, 2006). The brain cannot be aware of anything or perceive anything. What it can do is transform energy, and this energy transformation obviously affects how a person can be aware of something or perceive something, but it does not change the fact that it is the person and not the brain that can do these things.

Not only Damasio, but countless other leading neuroscientists excel at the mereological fallacy: Francis Crick writes that the brain has "convictions" and that it can "guess" and "interpret"; Gerald Edelman argues that the brain "categorizes" and "manipulates the rules" and Colin Blakemore writes that neurons 
have "intelligence" and "knowledge" and that they "present arguments" for the brain (Bennett \& Hacker, 2003, p. 68). This is just a small sample from a very large number of mereological fallacies in scientific literature. However, it could be argued that this is merely an innocent metaphorical way of expressing oneself; these scientists are well aware that the brain cannot do these things (which is actually Blakemore's response to the criticism), but scientists' theories and models are often dependent on their meaning what they say - literally.

One should pay attention to the fact that if it does not make sense to attribute psychological characteristics (paying attention, for example) to the brain, then it does not make sense to attribute their negations to the brain either. The fact that the brain cannot pay attention does not mean that it is therefore inattentive. The fact that the brain cannot see does not mean that it is therefore blind - in the same way as my oatmeal cannot be said to be either awake or sleeping! Again, this is not an empirical fact about brains and oatmeal, because we cannot someday discover that the brain can actually see, or that my oatmeal can actually sleep. Instead, it is a conceptual fact, i.e. a fact about how we can meaningfully use our language's psychological terms. Wittgenstein expressed this point as follows: "only of a living human being and what resembles (behaves like) a living human being can one say it has sensations; it sees: is blind; hears; is deaf; is conscious or unconscious." (Wittgenstein, 1953, §281). Our mental concepts can be applied only to living creatures with a certain range of behaviours - and the brain is not a creature in the required sense. It is an organ of such creatures.

So even though the brain is an amazing and fascinating organ, it is still an organ - and nothing else. Of course, the brain and the mental realm are linked, but so are the liver and the mental realm, for that matter. The psychologist Daniel Robinson, a current critic of brain reductionism, radically rejects the idea that a "neuro-philosophy" should be any more important than a "hepato-philosophy" (a liver philosophy) (Robinson, 2008, p. 210). I will not go that far. As a condition for mental life, the brain is an essential organ in a way that the liver is not, but Robinson is correct in that the mind is no more identifiable with brain processes than it is with liver processes.

\section{Materialistic perspectives on the brain and the mind}

In the previous chapter, I discussed some different perspectives on the mind divided into two main tracks in the history of ideas: the consciousness viewpoint and the rationality viewpoint. I argued that modern variations of the first main track are often concerned with understanding consciousness and thus the mind purely materialistically as a property of the brain (as simply as the brain as such). In this chapter, we will deal more thoroughly with the theories that link the mind very closely to the brain. This applies to the vast majority of theories in psychology, psychiatry and cognitive science nowadays, and my remarks above have endeavoured to identify why this is problematic. It is obviously not problematic to 
be interested in the significance of neural processes on psychological phenomena, but it is problematic to conclude that the mind is the brain. Hardly any psychologists or neuroscientists today are metaphysical dualists like Descartes. Nevertheless, as mentioned in the previous chapter, it can be said that many of them build on the premises that Descartes set out. They are often, in various ways, neural Cartesians or Cartesian materialists. Three major materialistic theories about the relationship between brain and mind emerged in the post-war era (see the overview figure from the previous chapter), i.e. three theories that have linked the mind very closely to the brain: the materialist identity theory, eliminative materialism and functionalism, all three of which will be discussed below.

\section{The materialist identity theory}

The materialist identity theory was first formulated by the Englishman U. T. Place (1924-2000) (1956). The theory is that the mind, in empirical terms, will turn out to be identical with brain processes. A significant aspect of the theory is that it is not presented as a logical or conceptual truth that a mental state is identical to a state in the brain (Bem \& Looren de Jong, 2006, p. 147). We cannot infer from the concept of a mental state alone that it must be identical to a state of the central nervous system (whereas we may well infer from the concept of "bachelorhood" that it refers to unmarried men).

Proponents of the materialist identity theory therefore believe that we have different concepts of mental states and physiological states. They also believe that it will turn out, as the neurosciences make further advances in the study of cognition, that mental states are equivalent to physiological states in the brain. At the same time, what is perceived as necessarily true is that (inner) mental states are the actual cause of (outer) types of behaviour. This can be said to be consistent with parts of "folk psychology", i.e. our everyday, common-sense understanding of mental life: humans, as psychological beings, have specific beliefs and desires (which are perceived as something "mental") that cause certain types of behaviour (perceived as something "physical"). This is mindism or a mentalistic view. My desire to eat strawberry ice cream, combined with the belief that I can buy the ice cream from the kiosk, causes me to move to the kiosk and make certain sounds that get the clerk to hand me some strawberry ice cream. The materialist identity theory then argues that both the desire and the belief are purely physical states of the brain.

Identity theory is based on an inner-outer dualism and places the mental realm unambiguously in the inner. There is a separation between the mental realm as something inner, in the form of beliefs, desires, experiences, etc., and the outer, which can be observed, including behaviour and movements. The inner is claimed to cause the outer, just as the outer can also affect the inner (e.g. if the kiosk is out of strawberry ice cream, which makes me buy chocolate instead). According to the theory, the inner is a part of the physical world. A classical example (within materialist identity theory) of a mental state being identical to a state of the brain, 
from an empirical point of view, is the experience of pain, which is believed to be identical to $\mathrm{C}$-fibres firing. This has subsequently been proved wrong from a neurophysiological standpoint, but this is merely an illustrative example so we will ignore that fact for now (Bem \& Looren de Jong, 2006). The problem is that, if there is to be identity between the experience (something mental) and the physical state in the central nervous system, then there must be identity between something that can be physically located (certain neurons firing in a particular place in the brain) and something else (the sensation of pain), which either cannot be located in space or can be said to be located somewhere other than where the neurons are firing. The point is that it is not your neurons that hurt when you cut your finger, but rather your finger itself. If this difference between the physical and the mental realms does exist, it is hard to understand how the two processes could be identical.

This kind of identity, which the materialist identity theory is about, is called type-identity. It is identity between a particular type of mental phenomenon and a particular type of neural process (e.g. pain and C-fibres firing). Uncovering this kind of identity relationship is an objective in many sciences; for example, temperature is type-identical with mean kinetic energy (Bem \& Looren de Jong, 2006, p. 159). Identity theory asserts, then, that a certain kind of psychological phenomena should be identical to a particular kind of neural phenomena. However, it has turned out to be particularly difficult to prove this assumption empirically. Neuroscience has simply not been able to demonstrate the kind of identity relationships that proponents of the theory thought might apply for philosophical reasons. And since identity theory does not postulate any conceptual link between the physical and the psychological, it is dependent on the fact that empirical, scientific proof of the identity relationship can be produced. Its lack of success on that point is probably one of the reasons for the materialist identity theory generally being abandoned today. Few scientists currently adhere to the theory.

Newer materialist theories are not concerned with type identity, but rather with what is called token identity, which states that we can describe only specific instances of mental events. Here it is merely argued that any instance of a mental phenomenon, for example when I calculate the square root of 25 , is at the same time an instance of a physical phenomenon, but the exact correlation between the mental and the physical can vary between species and individuals. This token identity theory leads to the view that the same mental process can be realized in different types of physical processes, which is a key assumption in the functionalist theory, as we shall see below. A calculator can calculate the square root of 25 as well as I can, even though we use very different machinery to do the job. I use my neural structure while the calculator uses its chips and circuits, but there is no doubt that some physical activity must take place in order for us - the calculator and me - to be able to make the calculation, each in our own way. A modern materialist would then insist that the calculation process, as an instance, is identical to a physical process - whether this occurs in the brain or in the calculator. 


\section{Eliminative materialism}

An extremely radical thesis about the brain and the mind called eliminative materialism was developed in the last few decades of the twentieth century (Churchland, 1981). In popular terms, the position is described based on the provocative assumption that there is no reason, as in identity theory, to discuss the identity relation between the mental and the physical, because the mental simply does not exist. And when something does not exist, it cannot be identical to something else! We should thus eliminate our concepts about the mind (hence the name of the position), as well as those of our explanations of behaviour that are based on psychological theories, and replace them with more tenable scientific concepts and theories, such as those found in the neurosciences. The most famous representatives of eliminative materialism are the husband-wife philosopher team, Paul and Patricia Churchland. Paul is said to carry a picture of Patricia's brain (from a brain scan) in his wallet instead of the usual portrait of her face, which seems to be quite a concrete expression of the idea that we are our brains! In their later writings, the couple have entered into partnership with empirical neuroscientists, especially from the so-called "connectionist" positions in cognitive science (more on this below).

Whereas the materialist identity theory can be said to respect folk psychology (that we have beliefs and desires that cause actions), eliminative materialism considers folk psychology to be an incorrect theory. According to eliminative materialism, there are no such beliefs and desires with intentionality, such as we envision from a purely folk-psychological perspective, and we must therefore replace the vocabulary of folk psychology with scientific vocabulary - and thus talk about our neurons firing rather than, say, our feeling pain. Briefly put, we should change our language to be more scientific so we can avoid referring to mental phenomena like beliefs and desires that do not even exist, and which therefore do not fit with a scientific world view whatsoever.

Eliminative materialism has been heavily criticized, and I will mention only a single point of criticism here: its attempt to replace folk psychological theory with a scientific theory rests on the premise that folk psychology is a theory in the first place, which is highly doubtful. Learning to use psychological terms in our language is not the same as learning a theory that can be falsified (Bennett \& Hacker, 2003, p. 369). For instance, when we learn to attribute mental states to other people (e.g. "he is afraid of the dog"), it is not the case that we are learning a theory that emotions (e.g. fear), in reality, are inner mental states that are separate from the behaviour that can be observed. The case is rather that we learn to use a term for the behaviour we can observe: the person recoils, avoids the dog, tries to protect himself with his arms, etc. Fear, in this case, is observable for anyone who has mastered the term "fear" and the correct use of the term is not based on any theory. Wanting to eliminate a theory that is not a theory to begin with is a problematic endeavour.

Eliminative materialism rests on the false premise that every explanation and all understanding is theoretical. When we talk about scientific theories, it is obviously 
reasonable to eliminate the false ones, but our everyday use of mental concepts is neither scientific nor theoretical, and it is difficult to imagine how human life should be lived without being able to use the explanations and understanding that our shared mental concepts provide. To understand how a body moves from point A to B, I can avail myself of physical causal explanations, but to understand why a person travels from his home to the local school to vote in the general election, I have to apply concepts that are retrieved from a system of intentions and reasons that cannot possibly be reduced to the way neurons fire (since neurons have neither intentions nor reasons to fire in a particular way).

By attempting to replace the everyday way of understanding and explaining actions with a more "scientific" way, eliminative materialism moves so far away from what it is really trying to explain that there is no longer anything to explain. For instance, if we want to understand the neurological basis for pain, which is a legitimate and important scientific ambition, then we can, for example, put people who are experiencing pain into a brain scanner, but in this case we are dependent on them being able to report in readily understood language that they have pain, in order for us to be able to interpret the scanned images in a meaningful way. In short, we have to rely on an intersubjective, "folk psychological" agreement about the concept of pain in order to be able to meaningfully read the pain's neurological correlate. This folk-psychological qualitative reporting of pain cannot be eliminated, because then the scientific project - which consists in identifying the brain's functioning - could not even get off the ground. If people could not tell us that something hurts, then we would not know when it would be relevant to scan their brains. Folk-psychological explanations and physiological explanations of behaviour do not represent competing theories on the subject, as is believed in eliminative materialism, because folk psychology is not a theory at all, but a way to give meaning to thoughts, feelings and actions.

Having said this, we also have to add that there are several current trends in our society that support and promote eliminative materialism in a way that risks making this opponent of folk psychology into a new - but impoverished - folk psychology. Biological psychiatry, for example, is rapidly growing and is close to becoming unchallenged as the explanatory system in psychiatry. Depressive patients today learn to say "I have low serotonin levels in my brain" rather than "I feel sad and empty". Parents can teach their children to say "it is my ADHD's fault" instead of "I am sorry I did not finish my essay".

I will not pass judgement here on whether these in particular are reasonable ways to express oneself or not (but see discussions in Brinkmann, 2016a; Nielsen, 2016). There is probably no doubt that a neurological vocabulary will increasingly affect our interpersonal relationships and our self-understanding in the coming years. And in my opinion there is also no doubt that, if this influence is large enough, it will have significant negative consequences for our ability to understand the depths of human existence. Even though depression is potentially accompanied by altered brain chemistry, and in many cases there is a lot to suggest that this is the case, not all forms of depression can be reduced to this dysfunctional brain chemistry. Anyone who reacts with depressive symptoms at the loss 
of loved ones in a traffic accident is reacting in a way that is deeply meaningful in an existential sense, regardless of how his or her brain is constituted. Anyone who has ever been in love knows that neurochemical vocabulary will never be able to replace the language that poets, authors and singers use to describe and express being in love. Although my objections to allowing neurological forms of understanding to have too great an influence on our self-understanding may seem old-fashioned and romantic, I do not think they are. I believe instead that they arise from hardline, irrefutable arguments about our need for multiple forms of language in order to be able to understand and express the multitude of qualities that our lives have. And when we deal with mental qualities, folk psychology cannot be eliminated.

\section{Functionalism and cognitive science}

The most prevalent perspective on the mind and the brain in psychology today is neither identity theory nor eliminative materialism, but rather what can be called functionalism in one form or another. Functionalism refers to all of the basic psychological positions that essentially consider the mind to be an information processing system, like a digital computer, where the idea of "mental representations" plays a key role. The reason that these ways of thinking are called functionalism is that mental states are perceived as having functional roles; they are causally affected by input from the outer world and have a subsequent causal relationship to other mental states and to outward behaviour (Bem \& Looren de Jong, 2006, p. 160).

In contrast to eliminative materialism, the view is that folk psychology is fundamentally right: thoughts, feelings, beliefs and desires exist, and there is therefore no need to eliminate references to these real, existing mental processes. And in contrast to materialist identity theory (as mentioned above), this point of view says that there is a one-to-one relationship between mental and neural processes but that the same mental process can be realized in many different physical systems (this was called token identity above) - and some functionalists would even go so far as to say that there is not necessarily a need for a brain for this purpose, but that a digital computer can form an adequate physical foundation for the existence of mental processes if it is designed in the right way. However, according to the theory there has to be some kind of material hardware in order for the mind to be able to exist as software (in this sense the viewpoint is materialistic), but it is not given what form this hardware must take. The basic view is that the function of the hardware (brain, computer or otherwise) is what is interesting, and not what the hardware is made of. The same function can be realized in many different materials, just like a mouse trap can be made in countless different ways and from various kinds of materials. As long as it fulfils the "mouse-catching" function, it is a mouse trap, regardless of how and from what it is otherwise constructed.

Functionalism is not just a philosophy, but is very closely linked with empirical and multidisciplinary cognitive sciences, which have experienced an explosive 
development since the mid-twentieth century, and which are often simply summed up as cognitive science. The cognitive science perspective of the mind is predominant in psychology in particular, but is also strong in linguistics, anthropology, sociology and the cognitive science of religion, and the paradigm is often so closely interwoven with the neurosciences today that the term cognitive neuroscience is used. Cognitive science in general and cognitive neuroscience specifically form the core paradigm in contemporary psychology upon which the basic empirical research and theory development has been based in recent years. Well-known psychological representatives include Stephen Kosslyn, Steven Pinker and Nobel laureate in economics Daniel Kahneman.

In a book about "the mind's new science", Howard Gardner (1987), himself a highly influential psychologist and the man behind the theory of "multiple intelligences", has written insightfully about the history of functionalism and the cognitive science paradigm, and he summarizes the following basic assumptions of the paradigm - or the "cognitive revolution", as Gardner calls it:

1 A first basic assumption is that we must work at a level of "mental representations" when investigating psychological phenomena and cognitive abilities. Mental representations are inner representations of an outer world, which exist at a level that is separate from the biological on the one hand and from the cultural on the other.

2 Moreover, a central premise is that the digital computer is the best model we have of the human mind.

3 Furthermore, cognitive science builds on consciously de-emphasizing certain factors at the expense of others. The fact that psychological phenomena have a physical and an emotional side, that they are influenced by historical and cultural factors, and that they are played out against the background of a given context is all set aside in order for researchers to be able to concentrate on the mental representations as such.

4 A fourth characteristic is the explicit multidisciplinary nature that cognitive science is based on (everything from artificial intelligence research to anthropology is relevant).

5 And lastly, Gardner points out that researchers are concerned with using empirical research to solve the epistemological problems that Western philosophy has worked with since the Greeks (Gardner, 1987, pp. 6-7).

Gardner's book is thirty years old, and as such it is a classic, but it is important to be aware that a lot has happened in the cognitive science paradigm since then. Not least, basic assumption number 1 probably needs to be revised since many researchers today would not sign off on the statement that the relevant level of mental representations is separate from, or independent of, its neural basis. Cognitive science, as mentioned earlier, has evolved into cognitive neuroscience, and many people are interested specifically in how mental representations exist in the brain. As mentioned in the section on eliminative materialism above, many 
people today work from a connectionist starting point, where the so-called "neural network" is assumed to be the basis for cognitive processes and where mental representations are understood as patterns in which the nodes in the network are activated like the brain's synaptic structure. However, the fact that the mind is perceived as a type of computer that processes information still applies today and the brain is perceived by analogy as an information-processing organ.

\section{Mental representations}

Despite the significant differences between classical cognitive science and newer connectionist variations, it is still the case that many of the fundamental problems that I will now point out in Gardner's five basic assumptions are also true of the more recent developments in the cognitive sciences. The most important problem concerns the very idea of mental representations. This is in many ways the central idea of functionalist thinking and research in cognitive science. Functionalists think that mental representations are, simply put, the contents of the mind. They do not think we are in contact with the world as such, but instead with mental representations of the world. We do not see a tree in the garden, but a mental representation of a tree in the garden. Nobel Prize winner in medicine Francis Crick (1916-2004), who discovered the structure of DNA, describes it as follows:

In perception, what the brain learns is usually about the outside world or about other parts of the body. This is why what we see appears to be located outside us, although the neurons that do the seeing are inside the head. To many people this is a very strange idea. The "world" is outside their body yet, in another sense (what they know of it), it is entirely within their head.

(Crick, 1995, p. 104)

True enough, this is a strange idea for many people, as Crick says, but in my opinion it is not because we are uninformed (as Crick insinuates), but because the idea itself is wrong. It is easy to imagine a psychiatric patient who has a delusion that everything that he sees is inside his head and not in the world. This "strange idea" would be a psychotic symptom - not a scientific insight, and it is not regarded as such until a recognized, Nobel Prize-winning scientist says it. Other neuroscientists similarly lock the perceiving subject inside the head. The famous neuroscientist Antonio Damasio writes, for example, that "when you and I look at an object outside ourselves, we form comparable images in our respective brains [. . .] Whatever it is like, in absolute terms, we do not know. The image we see is based on changes which occurred in our organisms." (Damasio, 1999 , p. 320). We are not in contact with the world, but only with representations (images) of the world, and when we think (according to proponents of the paradigm), we manipulate these mental representations, most often in the form of symbols, and it is the researchers' task to find the rules and algorithms, inspired by computer science, by which we manipulate the representations and process the 
information. This idea is also often followed by an application-oriented perspective that, for example, considers it likely that discovering the relevant rules and algorithms will enable us to optimize human learning processes.

In particular, the quotation from Crick illustrates the mereological fallacy that I mentioned earlier. It is nonsense to say that "neurons see" because neurons do not have eyes. But here I would like to investigate the very notion of mental representations. The problem with the depiction of the human mind that functionalism and cognitive (neuro)science is based on can be put very simply: cognitive science needs a concept of mental representation, but for something to be a representation, there has to be public use of it (Hamilton, 2008, p. 120). This is an insight that Wittgenstein (1953) formulated in his rejection of the idea that a private language could exist (see also Proudfoot, 1997). For a language to be able to have meaning there must be a reasonably consistent use of the language's words, signs and symbols. There has to be a difference between correct and incorrect use, because if a word is used completely at random, then it does not have meaning. Language is thus fundamentally normative; meaning depends on normativity. What other than a language-using community can define the meaning of words, signs and symbols? Wittgenstein's answer was: nothing. If the words in a language refer to something that only an individual speaker can access (a private world of mental representations, for example), then it would make no difference whether you use words correctly or whether it only seems to that person that he is using them correctly. So in that case, we cannot talk about correctness at all, because this presupposes a difference between correct and incorrect usage in the normative sense.

Since language is normative in the sense that it has meaning only by virtue of a difference between correct and incorrect usage, then for language to even exist at all there has to be a language-using community, i.e. something outside the speaker himself, which determines the language's normativity. Something can mean something only if there is a practice of using it in a particular way: a road sign does not mean anything in and of itself - it has meaning only because the sign is used in a specific and relatively consistent way by a community. So nothing means anything inherently and nothing has intentionality in and of itself; this is only the case against the background of a social practice that allows one to be corrected based on current norms. If mental representations are assumed to be like (inner) road signs in that they designate a particular form of behaviour as appropriate, or they represent something other than themselves, then they cannot be private. Therefore, the mind is in reality public in a sense that the theory of mental representations cannot capture. If a person is happy, smiling and whistling, then it is not a matter of him having specific inner representations that are translated into an outward expression. The outward expression is the psychological phenomenon of being "happy", or at least a significant part of it. And we can see that the person is happy by observing him directly - there is no need to scan the person's brain or anything along those lines to prove it. If we imagine that we nevertheless scanned the person's brain and found that it did not show the usual neural signs of happiness (but depression instead), then we would still have to maintain that the 
person was happy, if he was sincere and said he was happy and his behaviour also confirmed it. Should there be any conflict between the individual's ordinary folkpsychological expression of mental states on one side and neuroscientific studies on another side, then the everyday, folk-psychological criteria of psychological phenomena like happiness should in general trump the "scientific" and neurological criteria. This is a principal and essential point: folk psychology does not need to be corrected or falsified by science, although it is often affected by it.

Mental representations are supposed to explain how we can recognize something as being a certain thing. Simply put, the theory claims that the mind can recognize a beech tree as a beech tree, because we have formed a mental representation of beech trees. It can be argued that the reference to mental representations does not solve this problem, but merely serves to transfer it inside the person, because we can then continue to ask how the person can know that the inner, mental representation depicts a beech tree. If it is difficult to explain how we can recognize that an object in the outer world is a beech tree, why is it thought to be easier to increase the problem twofold and also have to explain how we can recognize that the inner mental or neural representation depicts a beech tree? This does not solve any problems; it merely creates new ones.

If Wittgenstein was right in saying that a sign or symbol can have meaning and thus represent something other than itself only because it is publicly used as such, then referring to an inner world of mental representations explains nothing at all. On the contrary, it merely makes the problem unsolvable, because the mental representations cannot mean anything in and of themselves. How can I know, for example, whether a given mental representation that "depicts" a heavy person is a mental representation of a pregnant woman or of a fat man? ${ }^{2}$ I can only "know" it by using the symbol in some way, and this requires a social context in which this usage can take place. There is nothing about an image of a heavy person in itself that determines whether it depicts a pregnant woman or a fat man - or anything else for that matter. It could symbolize a food lovers association or it could be an Egyptian hieroglyph meaning "God". No image, no symbol, no sign, no word determines its own interpretation. Significance and meaning can occur only in a particular social context of usage within a social practice. This makes the whole notion of inner, private mental representations highly problematic, because it seems that this notion presupposes an idea of private language usage, which, according to Wittgenstein's argument, is untenable. If meaning depends on contexts and communities, then an even more problematic issue is that a large number of cognitive science researchers are engaged in side-lining human life forms, cultural and social factors, as Gardner pointed out above. Life forms, culture and social interactions are precisely what constitute mental phenomena as such. True enough, the brain is one significant condition for the manifestation of mental life, but our bodies and social relations play an equal part, so there is no point in de-emphasizing them if you want to investigate the mind. The mind is not inside the head, but is linked to the fact that we as individuals take part in social practices where significance and meaning can occur. 
The "mental representation" way of thinking is prevalent in modern psychology and cognitive science as the fundamental view of the mind. The fundamental view, as has probably already become clear, is that the psychological realm consists of mental representations that can be processed and combined according to further specified algorithms and rules. These rules are often referred to as syntax, and the linguist Noam Chomsky in particular has advocated for a syntactic perspective on mental processes for years (Chomsky, 1966). In his linguistic writings, Chomsky uses a critique of behaviourism with regard to language learning as his starting point. Behaviourism is a theory that psychology should concentrate on studying behaviour, and that behaviour is shaped by reward and punishment and nothing else. Behaviourism works well as a practical tool for training dogs and can also be used to guide human behaviour in desirable directions to some extent. A great deal of everyday psychology is based on behaviourist principles, and in TV programs where psychological experts visit people in their homes to help them raise their children, they almost always recommend a behaviourist intervention. Such an intervention comes down to two things: rewarding the behaviour you find desirable (and a reward does not have to be a material thing - it can also be attention, for example) and ignoring the behaviour that you find undesirable. The undesirable behaviour will often gradually disappear. Behaviourism as a theory used to be quite influential in the world of research, but today it exists primarily because of its practical utility and not by virtue of its theoretical insights.

A basic problem with behaviourism as a theory was pointed out by Chomsky, and this contributed greatly to its loss of status in the world of science. If people learn languages in the way that behaviourists imagine, i.e. exclusively by hearing adults speak and being rewarded for imitating the adults' speech correctly, then it would be a never-ending process. In practice, however, children learn relatively quickly to construct sentences that they have not heard before, and they have an understanding of structurally complex sentences like: "The apple that is next to the book on the table that I painted last year is ripe." According to Chomsky and his successors, this requires that they have an understanding of grammatical structure, which can only be inherited. According to these researchers, children have a language instinct, an innate mental module for language learning, which is activated by the local language that they are exposed to in their culture. Chomsky's approach has had enormous implications even outside the field of linguistics, where particularly the idea of modules, i.e. specialized information processing systems in the mind, has appealed to many researchers, and cognitive scientists have postulated the existence of modules for a wide range of abilities, such as the capacity for moral reasoning, for example (Hauser, 2006). The basic idea is thus in contrast with that of the behaviourists; they believe that our mental functions are learned, whereas modern cognitive science believes to a much greater extent that we have innate modules for language, morality, sociality, and more. In recent years we have seen a return to the forms of nativism (a belief in innate qualities) that had otherwise been abandoned for many years. 
However, many have also been critical of the syntactic-modular approach to the mind in the tradition of Chomsky: the idea that the mind consists of modules that process information based on syntactical rules. The philosopher John Searle is a major critic. In a very famous article, Searle claimed that the approach cannot possibly account for the human ability to understand anything - not even to understand language (Searle, 1980). Searle proposed a thought experiment, known as the Chinese room. An English speaking person is imagined to be in a room with an inbox and an outbox. He is then given a number of inputs in Chinese through the inbox, and he can consult a manual where he can see which relevant characters he is to send back via the outbox. In other words, he receives Chinese characters and processes them based on a manual and then sends different characters back out. Searle's point is that, even though he can do this satisfactorily, we cannot say that he understands Chinese. He simply does not know what the characters mean, what they represent. The example is clear enough in analogy with cognitive science's view of the mind: the room is a language module, and the manual is to be understood as an analogy for the syntactic rules for combining linguistic elements, and Searle concludes that syntax is never enough to create understanding, because the English speaker in the room does not understand what he is doing. In order for a language to mean something to someone, the person has to participate in the life form in which the language is used, and this requires the presence of the whole person - both head and body - in a social community.

Searle's thought experiment has been fiercely debated, and regardless of whether it is valid in the details, I believe that it is a very fine, concrete expression of the functionalist view of the mind as an inner symbol-processing system that is only connected to the world (both the physical and the social) via narrow input-output channels. Functionalism and much of cognitive science can rightly be accused of locking human beings inside their own minds, where we only have access to mental representations of the world and not the world itself. Incidentally, we can also note that there have always been good alternatives to this view of the mind, in psychology as well, where John Dewey was an early representative of such an alternative (see Brinkmann, 2013). From the late nineteenth to the mid-twentieth century, Dewey developed a perspective on the mind in which human action plays the central role. Cognition does not passively copy the outer world into inner representations - it does something much more active: acting in the world in order to transform it to benefit the activities of human life. Cognition does not consist in passively filling the mind with information. Instead, cognition is a function of action; the typical cognitive situation is not one where some stimulus unexpectedly occurs in the presence of a passively observing person, but rather one where the person is actively trying to achieve a particular stimulus. People don't merely hear as a passive process; we listen as an active process:

If one is reading a book, if one is hunting, if one is watching in a dark place on a lonely night, if one is performing a chemical experiment, in each case, the noise has a very different psychical value; it is a different experience.

(Dewey, 1896, p. 361) 
The same sound in a physical sense has different psychical or mental qualities depending on the activity that the person perceiving the sound is engaged in. Cognition and experience arise out of human activity and do not have to do with representing the world - but mastering it. It can be stated more elegantly as follows: it is not about "copying the world" but about "coping with the world" (Rorty, 1980). The school known as ecological psychology also rejects the representational view of the mind in cognitive science and believes that we can best explain our perception of the world based on a notion of "direct perception" that is not mediated by inner, mental representations (Gibson, 1986). Just as we can often directly see happiness in another's face and behaviour, we can often recognize the meaning of objects because we actively explore the world on the basis of particular habitual patterns of action. If you have experience with eating in the imagined hemisphere of the West, then you can directly recognize the importance of knives and forks as tools to facilitate the intake of food. We do not need to compare the visual impression of the knife and fork with mental representations of these tools. Both pragmatism and ecological psychology break down the image of the mind, which leads us to introduce a level of mental representations to explain cognition, but these traditions have regrettably been minority positions in mainstream psychology.

\section{Qualia}

The reader may accept the inherent problems in cognitive science and the concept of mental representations discussed above, but what about the way it feels to see, hear, touch, smell and taste the world? Do we not need a concept for our experiences here? According to many consciousness researchers, what makes the mind problem so difficult is precisely the problem with these experiences, or qualia. Qualia is the term that philosophers and psychologists use to describe the very quality of experiences themselves, such as the experience of the taste of beer, the nuances in the colour of the sunset, or the tingling sensation I get when my arm falls asleep. Regardless of how many physical facts science has managed to state about the brain, central nervous system and our sensory systems, according to some researchers there will always be a subjective experience, a quale, associated with our recognition of the world, which cannot be described scientifically from an objective point of view, such as by describing the processes in our brains. In short, there is a way that it is like to have experiences that are irreducibly subjective (Nagel, 1974). There is no way in which it is like to be a brick or a river, since these objects are not conscious, but there is a way in which it is, or feels like, to be a bat, a dolphin or a human. This "how-it-is-like-ness" is perceived by some to be the main problem in consciousness research. The discussion of qualia has been central for a number of years and it divides people into two groups: those who believe that the subjective-experience perspective is of an entirely different nature than the objective-scientific perspective and cannot be reduced to this, and those who in contrast believe that a scientific perspective will also be able to explain the qualitative dimensions of our experiences. 
I believe that the most productive approach to the discussion is based on a conceptual analysis (Bennett \& Hacker, 2003). Doing so will rightly cause us to doubt whether we can meaningfully talk about there being a specific way in which it "is like" or "feels like" to hear, see or smell anything, apart from actually hearing, seeing or smelling something. The qualia problem is thus a pseudo problem that is based on false premises. If we ask a normal person about how it is or feels to see an armchair, an answer such as "splendid!" would not be out of place if the person was tired and wanted to sit down. But if we ask for an answer to the philosophical question about qualia, the person would probably not know what he or she should answer. There simply is no particular way it is or feels like to see an armchair. To believe otherwise is to be seduced by our problematic psychological depictions of the mind. The experience of seeing an armchair is of course different from seeing a coffee table, but this, as a matter of course, is because the two objects being observed are different, and not because we are dealing with two different qualia in the philosophical sense.

From an ordinary language analysis, we have to doubt that we can meaningfully talk about there being a specific quale associated with any given experience. If we are to speak meaningfully about qualia, we have to speak of them as properties of objects and not of experiences. When I see that there are grey clouds in the sky, then the clouds' greyness is a property of the clouds and not of my experience. It makes no sense to say that an experience is grey - any more than it does to say it is not grey. Objects can have colours, but mental processes cannot: "What colour was your experience?" is a meaningless question. The problem of qualia arises because of the Cartesian tradition, which constructs the relationship between the seeing and the seen as a relationship that involves the seen being copied and observed in an inner world. From the perspective developed in this book, the objective should not be to solve the qualia problem, but to dissolve it by showing that it is based on false and confusing premises.

If we start with Aristotle rather than Descartes, then the qualia problem does not even turn up. Of course we should not deny that our experiences can have qualitative characteristics. For example, they can be pleasant or unpleasant, amusing or boring, but this is a matter of a person's affective relation to experiencing something specific and does not concern a mythological quale. The phrase "the film was amusing" makes sense, whereas the phrase "my qualitative experience of the film was amusing" does not. When we talk about "an amusing experience", we are in fact talking about having experienced something amusing (e.g. a film) - we do not mean that we had a certain inner object - a quale or mental representation that was amusing. And when I see a red apple, there is only one object involved: the red apple - and not an experience of seeing a red apple. What I see is the apple, and not the fact that I see it. In reality there is no problem with qualia, at least not if we start from the right basis and avoid the Cartesian consciousness tradition's manner of approaching the questions. In short, we need to wean ourselves off of the bad habit in philosophy and consciousness theory of thinking about the mental realm as inner objects. If we wean ourselves of it, then it also becomes meaningless to try to reduce the mind to the brain. 


\section{Evolutionary psychology}

As psychology appears in the mass media, not only are biological explanations for human behaviour involving the brain apparently in demand, but evolutionary explanations are as well. It is not simply a case of trying to understand the psychological realm based on the brain's functioning, because we also want to know why the brain looks like it does. One response to this is that the brain's structure and form are the result of an evolutionary process. These days, no serious scientist would deny that Darwinian evolution has to be taken into account when analysing our mind, but the question is: precisely what role does evolution play? In recent years, a significant segment of functionalism and cognitive science has entered into a partnership with evolutionary psychology, which is interested in how mental capabilities developed in response to evolutionary pressures during human prehistory. Prominent researchers such as David Buss, Steven Pinker, Leda Cosmides and John Tooby have developed a type of evolutionary psychology, based on the basic premises of the cognitive science paradigm, not least of which is the premise that there are innate, mental modules that process certain types of information and result in certain behaviours. The idea is that the mind, just like a computer, is an information processing system organized into modules that developed as a result of evolutionary pressure (Smith, 2005). We act the way we do because of our modules, and our modules look the way they do because of our genes, which, when all is said and done, is the ultimate explanatory factor for the mind. This way of thinking about evolution is attractive to many - scientists, journalists and laymen alike - presumably because it is elegant, simple to communicate, and seems to be able to answer all kinds of question about the mind, from why men rape women to why we are so afraid of spiders but not electrical outlets, which are objectively much more dangerous.

Psychologists sometimes talk about these mental modules as "mental mechanisms". Katzenelson has described four of their properties: (1) They have their current form because they have been adaptive by being able to complete a particular task in life such as reproduction; (2) they are created to capture a certain small amount of relevant information that is significant for precisely this task; (3) the module's input information tells it which task is to be completed, upon which the input is transformed into an output; (4) the output can be physiological activity, information to other modules or behaviour (Katzenelson, 2007). We see that this entire input-output idea is akin to the theories that view the mind as a kind of advanced computer. It is a very mechanical approach to mental life. According to this theory, the mind is like a Swiss army knife with a number of separate tools (modules) that have developed because of evolutionary pressure to solve certain tasks (take down enemies, find a mate, care for the offspring, communicate with others in the group, etc.) (Bem \& Looren de Jong, 2006, p. 210). Like the tools of a Swiss army knife, the modules have evolved relatively independently, but the modules themselves (for language, morality, etc.) are perceived as universal for the human species. The mind thus has a universal, modular architecture, which is adapted to the hunter-gatherer societies in which our prehistoric brains evolved. 
As mentioned, this story has proved highly marketable in the media, which gladly provide explanations, based on evolutionary psychology, for fashion, morality, gender differences and much more - for example, that men are promiscuous "by nature" because they are designed to "spread their genes", whereas women are relatively more faithful because they are designed to be stable caregivers. Besides the fact that it is a bit odd how often explanations based on evolutionary psychology are consistent with the prevailing moral notions in American society (where most evolutionary psychologists are from) module-based evolutionary psychology also suffers from the same basic problems as the more computer-inspired segment of cognitive science: mental modules, syntactical rules and mental representations are scientific constructs that are not supported by actual neuroscience, which in reality does not give us any reason to believe that the brain is "modularly" organized (Hamilton, 2008, p. 107). If we cannot find clear evidence of mental modules in the physical brain, then we have only behaviour to go on, and this risks an instance of circular reasoning where we explain the module based on behaviour and behaviour based on the module. This circular reasoning is a general problem in evolutionary psychology: there is a risk of explaining the existence of a certain type of behaviour based on the idea that it was evolutionarily adaptive. And if we ask how it is possible to know that it was adaptive, the answer is that if it weren't, it probably would not have survived to the present day! Such an explanation obviously has no scientific value.

Not all evolutionary psychology makes use of such circular explanations, but you often see them: Why do women wear lipstick? Because it was adaptive in relation to attracting males, who are attracted to the health and sexuality that red lips represent. How can we know that? Because otherwise women would not wear lipstick! Besides the fact that this does not prove anything, there is undeniably a wide range of cultural differences that are ignored, specifically in regard to body decoration. Most cultures do not have lipstick, and black lipstick is worn in some sub-cultures (e.g. in punk communities), which is not exactly a sign of health, and other cultures have various ways of deforming the lips that are not attractive for the majority of the "Western world" (e.g. the Mursi people of Ethiopia). Evolutionary psychology has a way of forgetting the many factors in the human world that are not universal, but which are associated with specific cultural contexts.

My criticism here is not directed towards an evolutionary approach to the mind as such, and there are a number of interesting researchers working in the borderlands between biology, psychology and anthropology, who are also sharp critics of the computer-inspired model of evolutionary psychology, for example, anthropologist Tim Ingold (2011) and psychologist Alan Costall (2004). My criticism is directed against the specific, but highly influential, segment of evolutionary psychology that suffers from what we could call the "neo-Darwinist Cartesian problem" (Hamilton, 2008, p. 109). This problem involves combining a causeexplaining, nomic approach to human life with an exploration of the normative issues that evolutionary psychology is interested in (morality, language, social life, etc.). As previously mentioned, it is problematic to give mechanistic or nomic explanations for normative phenomena. This is not to say that I deny there is an 
evolutionary basis for the mind. Since Darwin, we have known that nothing in the biological world is permanent and that the mental capabilities and dispositions of the human species also need to be understood within an evolutionary framework, broadly speaking. The problem does not occur until you try to make evolutionary explanations the only valid explanations in psychology.

A more productive perspective is to say that the mind is not a "thing", but a range of complex abilities and dispositions, and then the mind can be said to be unique among the products of evolution, since people, by virtue of their mental capabilities, are not just passive victims of the randomness of evolutionary history, but are also capable of influencing the course of history. The fact is that man is a co-producer of his own living conditions, a fact which Karl Marx made the cornerstone of his philosophy. Also other animals do this, of course. Just think of spiders or ants that create their own ecological niches. Darwin even described earthworms as being organisms that have affected and created their own living conditions (the soil) just as much as they have adapted to their environment (Costall, 2004). Earthworms are a product of the soil, which in turn is a product of the earthworms' activity, and so on in the cycles of life. Organisms and the environment have evolved together in a reciprocally influential relationship. But humans do this by using language, signs and symbols, and this brings us into the world of culture proper, which is why we have to supplement evolutionary explanations with cultural psychology. The cultural world is the manufactured world, which is not handed down through genetic material, but through education, socialization and reproduction of society. This is the subject of Chapter 4. All this should remind us that we should not think mechanically about the mind as a fixed evolutionary product, but rather as a range of abilities that enable us to transform and reconstruct the world we inhabit.

Giving evolutionary explanations a monopoly is problematic for the science of the mind, but it is even worse for our lives in general if we begin to understand and explain our own and others' actions in light of evolutionary psychology. I recently read in the newspaper that some psychologists believe that lying has evolutionary advantages. Supposedly, this is why we lie, and that is why these researchers believe that lying is excusable in many cases and perhaps even justifiable. If people adopt such a view, they might manage to convince themselves that lying is okay - that is what evolutionary psychologists say, right? - and this would undoubtedly be detrimental to social life, particularly because it will destroy trust and loyalty between people. And this is just one example of how scientific methods for understanding the mind have found their way into our self-understanding in recent years. This is not just plainly bad - it can also make it difficult for us to understand the depths of human existence if it is exaggerated.

\section{Conclusion: What is in our heads?}

"There is nothing in your head other than your brain!" sociologist Harold Garfinkel once said (see Watson \& Coulter, 2008). This chapter has presented an understanding of the relationship between the brain and the mind that essentially 
shares this premise. There is no mind inside the head. There is no "inner world", as we can plainly see by opening up the skull. When we open up the skull we see connected neurons that fire in complex patterns, but we do not see any mental modules, thoughts, feelings or memories. We should not think about the mind as a thing that is inside the brain. The relationship between the mind and the brain is not like the relationship between the heart and the muscle tissues that the heart is made of. The heart is made up of muscle tissues, but the mind is not "built up" of brain tissue in the same way. To continue with the same metaphor, it is rather like the relationship between the heart and the heart's job, which is to pump blood around the body so that cells can get oxygen. The mind is a range of rational abilities, and these cannot find expression without a brain, but that does not mean that the abilities can be reduced to the brain, or that they are the brain, because we cannot meaningfully say that abilities "are" an organ.

Although it is philosophically problematic to reduce the mind to the brain, this is nevertheless an endeavour that has been widespread throughout philosophical circles in Western societies in recent years. We initially looked at Lone Frank's book as a well-known example in little Denmark. However, only few researchers have elucidated what happens when bio- and neuro-scientific insights circulate throughout society today and leave their mark on people's self-understanding and on social practices. Some of them, however, are working on it. One example is the psychologist and sociologist Nikolas Rose, who in his earlier works was interested in how psychology's forms of knowledge and practices "subjectivize" people in certain ways - i.e. how they create subjects that are autonomous, self-managing and self-evolving. In his most recent book he has shifted his focus to bio- and neuro-sciences, arguing that future subjectivity-forming knowledge will come from these fields (Rose, 2009; Rose \& Abi-Rached, 2013). The anthropologist and ethnologist Emily Martin has similarly worked on psychology's role in American society, and she is now working to understand the meaning of the neuroscientific segments of psychology, among other things (Martin, 1999). She asks what sort of people neurologically based theories of the mind will foster. Her answer is that there are three likely consequences: (1) we will believe that our behaviour depends on our brains and not sociality, (2) we will believe that our brains are designed as an evolutionary product of a fight for survival and (3) we will believe that we essentially share the same neural structures (p. 582). This is not to say that all brains are equal, but that common structures form a background against which individuals vary. For example, a widespread educational theory is that we have different learning styles because of our brains' differing abilities, which is why we should be given different kinds of learning assignments. Martin combines the spread of a neuroscientific approach to the mind with societal trends in network capitalism, where major social and government institutions lose importance and where individuals become nodes in the network (the brain is a frequently used metaphor for understanding the social sphere via the network metaphor). Our understanding of the mind has been influenced in recent years by our understanding of the brain - and both have been influenced by the way we have organized modern society. 
Martin predicts that the built-in normativity in this view will mean that we will no longer spend time participating as much in public ways of life, but rather striving for individual improvement of our own minds based on knowledge of their functioning. We will be (even) more concerned with ourselves when we, in Frank's words which I quoted earlier, recognize that we have a "responsibility for our own brains" - but we may not feel responsible for very much else. We risk coming to believe that "society" does not exist, and there would therefore be no reason to care about it. Everything is in the brain! Rose's conclusion is similar: although the humanistic psychologists' core self has been replaced by the brain in recent years, self-realization is still the dominant cultural imperative for individuals. Perhaps the goal is no longer to "find yourself" but, again to quote Frank, "to increasingly be able to choose yourself or mould yourself in the direction you want. Again, it is a message of liberation: your biology is not a prison but a lump of clay that you yourself can contribute to shaping." (Frank, 2007, p. 301; my translation). Various bio- and neuro-medicinal options for self-improvement will probably increasingly be used not only to treat the mentally ill, for example, but they will also be used by "ordinary people" in the form of various drugs to make themselves smarter, better focused and - maybe - happier. Rose asserts that we have become "neurochemical selves" and that the future of self-improvement will be practiced as brain development, and Martin concludes that neurology is colonizing our self-understanding. If these diagnoses are even only approximately correct, then it is all the more important that we take care to understand the relationship between the brain and the mind properly. The fact is: you are not your brain. You have a brain, which is centrally involved in whatever you do. But you as a person are the doer; not your brain.

\section{Notes}

1 Politiken, January 17, 2009.

2 We are assuming here that a representation is figurative or "iconic", which is just one theory of mental representations (see Gardner, 1987). 


\section{Chapter 3}

\section{The mind and the body}

An image of the mind is beginning to take shape: if the mind is not a thing, but a range of abilities and dispositions, then we cannot say that the mind is inside our heads. In fact, attempting to localize the mind at all is problematic and a more productive strategy is to examine the conditions under which what we call mental abilities can find expression. We saw in the previous chapter that the brain is one such condition. Without a brain there is no mind, so the brain is a necessary condition for the existence of mental life. Is it also a sufficient condition in itself? No, because we can only say that living human beings, or things that behave in a human way, have sophisticated mental properties (Wittgenstein, 1953, §281). A well-functioning brain does not behave like a living person, but like the organ that it is. We do not attribute mental properties to a brain outside its physical body, such as a brain in a vat, for example. Our mental concepts can "grab hold" only when we are dealing with a moving, acting and explorative body. We need to have a self-propelling body that actively moves around in the world in a meaningful way. In order for mental abilities to be able to exist and manifest themselves, we need a body. The body is an essential condition for mental life. As Aristotle put it, the mind is simply the form of the living body.

The dualism between the mental and the physical is central to Christianity and Western culture. From this perspective, the body is viewed as an object, as part of Descartes' "extended things", whereas the mind is a subject - the acting, thinking and feeling factor that is imagined to "inhabit" the body in some way, perhaps as a kind of "ghost in the machine", to use Ryle's apt phrase (Ryle, 1949). A great deal of current philosophy is concerned with breaking down the walls between the body and mind. A good example is Mark Johnson, who talks about the body as the "body-mind" in an attempt to overcome the terminology of our problematic dualism (Johnson, 2007). Many adults in the Western world may sometimes have the feeling that the mind is an incorporeal and disembodied thing - for me, when I think about it, it feels a little like my mind is located about three centimetres behind my eyes. But from the outset, there is no doubt that from birth we are beings in motion; our physical being is absolutely essential. Foetuses are already moving in the womb; they orient themselves in their little worlds using their movements, and immediately after birth they start moving towards their mother's breast if they are 
laid on her stomach. As physical beings, we are in motion, and motion provides our basic access to the world. We can say that the first and fundamental manifestation of the mind is as movement and not as passive observer. We do not attain a capacity for cognition of the world in a broad sense by being spectators to it, but only by participating in it. Our mind, like our ability for cognition of the world, is inextricably linked to our ability for physically moving, acting, behaving, doing.

In this chapter, we will look at the body as a condition for the existence of the mind. Like the brain, the body is a necessary condition - a mediator (Brinkmann, 2011b) - for mental life. Unlike some of the classic theories in cognitive science that were mentioned in the previous chapter, we cannot say that a computer has a mind, because it lacks above all the ability to move in meaningful ways motility - associated with having a biological, flesh-and-blood body. I will first give a brief - and by its very nature simplified - introduction to phenomenological philosophy, which forms the basis for many of the current attempts to rethink the body as something more than an object. The body is also an object, and can be studied as such by medical science, but it is first and foremost intimately connected with our subjectivity, and is as such the basis for our cognition and creation of meaning in the world, which I will also touch on. Finally, I will look at the concept of habits in this chapter, since habits are closely linked to the body and the existence of mental life as such.

\section{Phenomenology of the body}

The French philosopher and psychologist Maurice Merleau-Ponty (1908-1961) was the modern thinker who most explicitly attempted to overcome modernity's objectification of the body with his phenomenology of the body. Modern medicine viewed the body as a piece of machinery that was somehow almost magically animated. As mentioned, it is not wrong in itself to regard the body as a mechanical object. It is one perfectly legitimate approach to the body if you are interested in getting a broken bone to knit back together properly; the processes involved here are causal and nomic. But the body is more than just a biological organism. It is, above all, also a "lived body", as Merleau-Ponty insisted. That is to say that we act in and have experiences of the world through our body. When you move your arm to pick something up, the experience is not one of first making the decision with the mind and then moving your body's arm as two separate processes. No, you move your arm when you want to; the will to do so, in a certain respect, resides within your arm. My arm, as a part of my body, is also a part of me as a subject - it is "mental" in a sense. This phenomenological experience of being your body is part of what is meant by "the lived body".

According to Johnson, we need five different ways of looking at the body in order to get a full understanding of it (Johnson, 2007, pp. 275-277):

1 The body as a biological organism: this is the body as it is studied by physiology and anatomy and which is healed by medical science. It is the body when 
viewed from the perspective of nomic sciences that explain processes causally, and it can function more or less well (there is thus a normative dimension here), but it is not the body itself that we praise or blame for functioning in a given way, but rather the person who possesses the body without being identical with the body. When viewed as a biological organism, the body is different from the person and from the person's mind. People have the right to vote - not their bodies. When we view the body based on traditional scientific methods, we turn it into an object and cannot fit it into our system of experience (Merleau-Ponty, 1945, p. 63). When we view the body as a biological organism, we look at it as a collection of tissues and organs, and this is a useful view of the body for a variety of purposes - when we have to perform surgery on it, for example - but it is a completely inadequate view when we want to understand our human and meaningful "lived" presence in the world.

2 The ecological body: Here we see the body as connected with its environment the ecology. Although we commonly think that we can easily differentiate the body from its environment using the skin as a boundary, this is actually a somewhat arbitrary and problematic notion (Mol, 2008). For example, the body depends on nourishment and oxygen, which are constantly moving into and out of it, and a large part of the body is made up of micro-organisms, which are independent beings that "live" inside of us, in a manner of speaking. In addition, the tools that we avail ourselves of can be said to be a part of our body. There are outright body prosthetics, of course, but there are also things like the blind person's cane or the visually impaired person's glasses, which can, with a certain degree of justification, be said to belong to the body - at least when viewed as an ecological body that stands in close relationship with its surroundings. We will look at this more closely in Chapter 5, when I explain the significance of things for the mind. Johnson concludes that the body is not separate from its surroundings, and that any boundary we draw between them reflects our current attempt to support some particular point (Johnson, 2007, p. 276). In short, when viewed ecologically, we must consider the body and the environment as two aspects of a single continuous process. Although we cannot yet explain human experience and complex mental life using this perspective of the body, it does provide a much more nuanced picture of the body than as a mere biological organism. Thinking of your physical being as closely linked and even confluent with your surroundings can also provide an aesthetic and almost religious experience: when we eat and drink, for example, we invite the world into our bodies. We receive the world within ourselves, and by reflecting on this we can experience an emotional resonance with the world, which, through these everyday processes, appears as a nourishing place, a place that we owe our existence to (Krueger, 2008, p. 608).

3 The phenomenological body: the rest of this chapter will deal with this way of looking at the body in particular. This is our body as we live and experience it, where it forms the basis for our experience of a meaningful world and 
therefore for our mind as well (Johnson, 2007, p. 276). The phenomenological body is the body as we know it prior to the scientific theories that we can learn about it (e.g. theories about the body as a biological organism). In other words, it is the body as it is experienced pre-reflexively and pre-theoretically, and this dimension of experience is precisely the field of interest in phenomenological studies.

4 The social body: The body is an element in a social world and not just in a physical or biological one. The body and its dispositions are shaped by the social interactions we enter into with others, from the time we are born and until we die. We are social and intersubjective beings from the beginning, and recent psychological research on infants and their interactions with caregivers has uncovered some of the ways in which the body and its habits are formed through early, complex patterns of interaction (a classic source is Trevarthen, 1993). This perspective on the body is related to the others, especially the phenomenological perspective. For example, the phenomenologist Iris Marion Young, in an essay entitled "Throwing Like a Girl", describes how young girls (at the time) in the United States were socialized to act in both the physical and social domains (Young, 1980). This was highly restricted in comparison with the boys' ways of acting. Young illustrated this point with a phenomenological description of the manners in which girls and boys throw a ball: While boys "occupy" the space with their bodies when they throw by swinging with their entire body, girls primarily move their elbow joints and their posture is much more restricted. In the United States this is known as "throwing like a girl" and it does not allow for the powerful throws we see in American baseball. For Young, it was an expression of the more general way in which girls and women are taught to be reserved. They are not allowed to be assertive in the physical or social domain. Social gender inequality is therefore reproduced not only as a difference between the sexes in terms of thinking and feeling, but all the way down to the level of bodily habits and patterns of movement. This is possible because the body is social. It is not formed through constant exchanges with only the physical world (as in the ecological perspective on the body), but through exchanges with the social world too.

5 The cultural body: This body perspective is closely related to the previous one, but it focuses more on the cultural differences that exist regarding appropriate and inappropriate ways to behave bodily. The body is always and unavoidably enmeshed in a network of standards that govern appearance, size, clothing, decoration and movement. There are cultural differences in masculine and feminine ways of moving, but also in terms of the "taste" that we unthinkingly react to positively from a physical point of view. The anthropologist and sociologist Pierre Bourdieu (1930-2002) identified several of these differences within French culture (Bourdieu, 1980). Taste is linked very closely to how the body reacts, for example, to what we respond to as erotic, beautiful, delicious, etc., and such ways of reacting vary culturally and also 
based on an individual's socio-economic class. Note, for example, the deep class differences that can be expressed in a sentence like: We sauté our potatoes in olive oil; they fry their chips in vegetable fat (Paterson, 2006, p. 46). Culture is not something that affects the body from the outside, but the body is in essence cultural by virtue of its way of appearing, posing and gesturing, and in its taste, which we can "sense" directly in our bodies.

As mentioned, we will focus primarily on the phenomenological body in this chapter. Phenomenology is linked to its originator Edmund Husserl (1859-1938), who wanted to create a philosophical method to describe the world as it appears to us prior to creating scientific theories about it. Phenomenology is the science of phenomena and the term comes from the Greek phainomenon, meaning "that which appears". Husserl described the mind's basic characteristic as intentionality, meaning that any mental phenomenon is characterized by being directed toward something other than itself. Phenomena always appear to consciousness. The discovered and experienced world is the world in which man originally finds himself. Husserl called this world the "life-world". The world is not what "I think", states Merleau-Ponty (1945), but what I "live through" (p. xviii). Here we turn away from the view in the representational way of thinking, where cognition is a passive or receptive process of "thinking" about the world, towards a view in which we recognize the world through "living" in it as fully corporeal beings (Dreyfus \& Taylor, 2015).

Part I of Merleau-Ponty's Phenomenology of Perception is about the body, and it states that humans cannot observe their bodies as objects for the simple reason that it is because of the body that objects can possibly exist for man (MerleauPonty, 1945, p. 105). What does that mean? On the face of it we might say that objects are objects - no matter how our body and mind might otherwise be constituted. Most people naturally have a rather unexamined relationship with objects like medium-sized objects in time and space, perhaps with furniture as a prototypical example of an object. But upon reflection, it becomes clear that the object concept is more relative. Not least, it is relative to an organism's size, shape and ability. For example, a blade of grass is an object for a fly that invites it to sit down on it in a way that does not apply to a human. And a microscopic parasite may relate to a person's skin as a landscape of easy-to-penetrate channels, while a fly cannot penetrate the skin but would rather land on it (Johnson, 2007, p. 47). An object is always an object relative to a perceiving creature.

The fact that objects are relative to the organisms that interact with them means that, to the same extent, it is the organism's constitution and behaviour that designates something as an object in the world, as it is the world's "objective" arrangement that determines how organisms act. In psychology this basic view is called "mutualism" since it is based on the fact that there is a reciprocity or mutuality between organisms (subjects) and the environment (objects) (Still \& Good, 1998). The psychological realm exists in this mutuality and not in either of the poles in isolation. This view is further complicated by the fact that this reciprocity is not 
static or permanently set in stone. It has dynamic or historic dimensions - not least in terms of evolutionary history; I previously mentioned Darwin's observation that earthworms have shaped their environment to the same extent that the environment has shaped earthworms. This is all the more true in cases where we deal with an advanced species like humans; we shape the conditions under which we live to a very great extent - and are, of course, conversely shaped by these conditions (Sterelny, 2012).

Merleau-Ponty analyzed the ability to move as the fundamental form of intentionality, and unlike Descartes he did not understand consciousness from an "I think" (". . . therefore I am") perspective, but from an "I can" perspective. Essentially, we are physically acting beings who acquire the ability to think reflectively only as something secondary. Our mental ability for thinking abstractly and reflectively itself stems from, as we shall see below, a certain physical beingin-the-world. "My first understanding of reality", say philosophers Dreyfus and Taylor, "is not a picture I am forming of it, but the sense given to a continuing transaction with it." (Dreyfus \& Taylor, 2015, p. 70). Our conscious intentionality, including our ability to reflexively want something, is derived from a more primitive and original transactional and operative intentionality that "produces the natural and antepredicative unity of the world and of our life, being apparent in our desires, our evaluations and in the landscape we see" (Merleau-Ponty, 1945, p. $\mathrm{xx}$ ). Thanks to our bodies, there is generally a naturalness in our dealings with the world. If we open a door to an unknown room and go in, we do not feel, for example, any urge to check whether the room has a floor, because perception is a bodily activity directed at the surroundings, and the majority of it takes place naturally and without reflection (Vetlesen \& Stänicke, 1999, p. 205).

The human being taken as a concrete creature is not a mind "attached" to an organism, according to Merleau-Ponty. Instead, the lived, phenomenological body - with its patterns of movement, reflexes and habits as a "body-mind" - is the entity that gives us experience of the world. Fundamentally, the body does not move because a disembodied mind has perceived something or seeks to achieve some specific objective. Instead, perception and cognition are functions of the body's movements and actions. This is also a key insight in James Gibson's ecological psychology, which in many ways is reminiscent of both Merleau-Ponty's phenomenology of the body and Dewey's pragmatism (Gibson, 1986). Through psychological experiments in perception, Gibson showed that we gain knowledge of an object's properties in time and space through the way we move in relation to the object. Information about the properties of the world is, in a manner of speaking, contained within the world itself, or at least in how a cognizant organism stands in relation to the world (again, a mutualistic principle), and not in inner mental representations which, according to Gibson, are superfluous in terms of explaining perception. Our recognition of the distance between objects, for example, can be explained by the way we move in relation to them. If we move to the side while looking straight ahead, things that are far away will not move as much within our visual field, relatively, as things that are close to us. These 
visual relationships alone allow us to recognize the location of objects in physical space. We do not need to introduce an illusory "inner computer" that is assumed to calculate these distances, precisely because they can be recognized without the use of mental representations.

Merleau-Ponty agrees that there is a need to reject the idea of mental representations. He says that consciousness has no "private life" (Merleau-Ponty, 1945, p. 32). As psychological beings we are always out near things and when we understand other people's minds, for example, it is not the case that we are interpreting their purely "outer" expression of supposedly "inner" factors. When we see an angry person, we do not see a "psychic fact hidden behind the gesture" that makes us merely think of anger. No, we see the anger itself directly in the person's expression. Nor is thinking some "inner" thing that exists independently of the world and words (p. 213). We think, feel, act and understand each other in the world - there is no veil between us and the world, which also means that there is "no privileged self-knowledge, and other people are no more closed systems than I am myself" (p. 393). In this way, we are surrendered to each other in public. The mind is not an inner thing. Since the mind is largely associated with the phenomenological body and since this body is in a world that is in principle public and intersubjective, then it follows that the mind itself is expressed in a public dimension that is not confined within a mythological inner world.

\section{Meaning, body and metaphors}

It is perhaps not so difficult to accept the argument from phenomenological studies of the body that fundamental intentionality, our primary way of recognizing and acting in the world, is embodied. It is easy to convince ourselves that the most "primitive" aspects of our mind are bodily. If you do not have a very strong theory about innate systems for cognition of the world or about a disembodied rationality, then you have to work with some kind of theory about our access to the world being via the body - that it is because of our bodies that we even have a world in the first place. At a basic level, most can understand that we thus need to start from an understanding of the mind as a "body-mind". But at first glance, what is more difficult to understand, based on the body, are the more advanced mental functions: our conscious memory of past events, our ability to speak and reason, our ways of reflecting on our lives. It is one thing that our actual psychological connectedness to the world is physical in nature, but what about our capacity for abstraction as expressed in intellectual thinking, philosophy, religion, mathematics, etc.? Many people will argue that it is precisely here that we see the specifically human way of being a creature with a mind. Animals are also tangibly connected to their world in a physical way (even earthworms), so in order to understand what distinguishes the human mind, we have to understand man as a creature that can think abstractly. The human mind is rational, as we previously emphasized, starting all the way back with Aristotle. We do not simply react immediately to any stimulus we are faced with, because we can apply concepts and reflectively consider our 
desires and beliefs. We can consider whether our current desires are worth having, and whether our beliefs are based on sound reasons. We are not just animals, but rational animals. Can our rational powers be contained and comprehended within a body-phenomenological perspective?

This is an open question, and it is also one of the most interesting questions in the current debate about the relationship between the body and the mind. The psychological realm is not understandable from the perspective of an embodied operative intentionality alone; it is also a complex normative dimension, as we have seen, and above all it has to do with meaning. One of the researchers who has worked out a convincing (in many ways) defence of the idea that our more abstract mental abilities can be understood based on the body is Mark Johnson, mentioned previously. In his book The Meaning of the Body, he develops a coherent theory about the "body-mind", which aims to prove that the body is the basis for meaning, abstraction and reflection. The body is thus what makes it possible for something to appear meaningful - it enables the experience of meaning and also allows us to create meaning in our lives, both personally and socially. Johnson goes so far as to say that both logic and reason are embodied dimensions (Johnson, 2007, p. 102). Throughout the book he develops a complex theory not only about the body, but also about the brain and its "neural maps" (which Johnson does not regard as mental representations) as well as what he calls "image schemas", which are dynamic patterns of interactions between organism and environment that function outside of consciousness. But the basic leverage behind his theory of logic, reason and abstraction as embodied (or body-mind) abilities, is a theory about metaphors. It is above all metaphors that enable us as bodily beings to go from meanings, which are in an immediate sense bodily based, to abstract thought (p. 176). Johnson's theory, which he developed in collaboration with the linguist George Lakoff in particular, involves the very strong and radical thesis that all theories and abstract concepts are metaphorically defined (e.g. Lakoff \& Johnson, $1980 ; 1999)$. The mind in this sense is a bodily process that enables recognition of the world based on the metaphors it creates. Let us take a closer look at this thesis.

\section{The body's metaphors}

What does it mean that theories and concepts are metaphorically defined? A metaphorical understanding is one in which we understand one phenomenon in light of another even though there is no literal connection between the two. In the previous chapter we encountered the metaphor "the mind is a computer". Scientists may disagree on many issues related to the mind, but even the most stubborn computer functionalists will probably not say that the mind is literally a computer. The mind does not use AC power, it does not have a keyboard, you cannot connect a printer to it, it cannot access the Internet, and Bill Gates does not have a near monopoly on its operating system. Computer functionalists will instead insist that it is productive to think about the mind as if it were a computer, just as it is productive to think metaphorically about psychological intimacy as physical proximity and 
purposeful activities as movement. We can say "the two of them were very close" without indicating that the two people lived on top of each other in a physical sense. But concrete physical proximity is a source of metaphoric meaning for the more abstract area that relates to psychological intimacy. Similarly we can say "she took the first step to becoming a doctor," even if the person did not physically move a muscle. Here, travelling in physical space is the metaphoric source that structures our understanding of engaging in a purposeful activity. Many other ways of talking about these things support the notion that our perception of psychological intimacy and purposeful activities are built on metaphors relating to physical proximity and movement, respectively.

We can go a little deeper with a third example using a key mental phenomenon. We have the metaphor: to understand something is to see it. When you understand something (e.g. in mathematics), the understanding is not literally linked to seeing something, yet we say metaphorically "do you see what I mean?" or "can you see the logic?" The point is that it is an immediate, concrete and bodily based activity (seeing) that forms the background for our abstract notion of what it is to understand something. The source domain of the metaphor in this example is vision, while the target domain is understanding. An analysis of this metaphor for understanding can be made from this as follows (from Johnson, 2007, p. 165):

\section{Source domain (vision) $\rightarrow$ Target domain (understanding) \\ The seen object $\rightarrow$ Idea/concept \\ To see an object clearly $\rightarrow$ Understand an idea \\ The person who sees $\rightarrow$ The person who understands \\ Light $\rightarrow$ The "light" of reason \\ Visual focus $\rightarrow$ Mental attentiveness \\ Visual sharpness $\rightarrow$ Mental sharpness \\ Physical standpoint $\rightarrow$ Mental perspective}

Such an analysis of our visual metaphor for understanding may seem banal, but if we examine the importance of this metaphor in Western culture's philosophy, education and science, it is anything but. Metaphors for cognition involving vision and light have permeated our thinking ever since Plato and have been critical for how we have developed essential social institutions, not least within the world of education (Brinkmann \& Tanggaard, 2010). People understand and learn, we believe, when they are "exposed" to a "visual impression" of what they need to learn about. Here we are dealing with one of the most important metaphysical background factors for the educational system, which plays a key role in modern society reproducing itself. Children must sit down and learn. They must be "enlightened" to continue with the metaphor. Johnson's point is that all of this stems from an immediate bodily, lived experience of vision. He would say that the abstract concept of understanding, metaphorically speaking, can be traced back to our experience of seeing something, to strive to see something more clearly. Our abstract concept of the mental process, that is, trying to understand, 
is metaphorically structured based on the concrete experience of trying to see something.

Another point Johnson makes is that this metaphor for understanding is essential, but it is not unchallenged. There are competing metaphors and if these had been dominant, a lot of things could be very different, for example, in our education and teaching practices. Another common metaphor for understanding is to grasp something. A schematic analysis of this metaphor can be made as follows (from Johnson, 2007, p. 166):

\section{Source domain (grasp) $\rightarrow$ Target domain (understanding)}

An object that is grasped $\rightarrow$ Idea/concept

To grasp an object $\rightarrow$ Understand an idea

The strength of the grasp $\rightarrow$ The depth of the understanding

To lose one's grasp $\rightarrow$ When understanding fails or disappears

Object out of reach $\rightarrow$ Incomprehensible idea

In the same way as above, there is a logic in the source-target domains here, but different bodily "image schemas" are activated when we think of understanding based on the metaphor of "grasping" rather than on the metaphor of "seeing". On the one hand, it has given rise to a hands-on teaching style, where learning is linked to actively working with and manipulating materials - exemplified by Dewey's famous motto "learning by doing" (Brinkmann, 2013). This hands-on teaching style recommends, for example, that students learn geometry by concrete experimentation with three-dimensional shapes that can be manipulated. And on the other hand we have a visual teaching style, where one imagines that learning can take place by students "seeing" materials, for example, by reading about them in a book or by watching the teacher demonstrate geometry on the board, rather than by obtaining first-hand knowledge. Both metaphors for understanding stem from bodily experiences, but they derive their meaning from two different ways in which we have concrete experiences. The radical thesis here is that all of our nonliteral concepts, theories and ideas derive their meaning from being metaphorical extensions of a certain type of bodily experience.

The above analyses of the two metaphors for understanding relate to what are called primary metaphors. Primary metaphors are based on an immediate experiential correlation between a particular sensory motor domain and another domain that has to do with subjective experience (Johnson, 2007, p. 178). Most of us have had such an experience, where trying to see something more clearly correlates with the understanding of what we see. For example, we are familiar with the experience of "decoding" ambiguous images that can be seen as two different things (but not both things at once), and seeing them involves trying to understand what they represent at the same time. We have also experienced grasping a thing in the literal sense, such a tool, and in grasping it we have come to master the tool as a correlation to understanding it. Johnson mentions other glaring (note once again a visual metaphor - they are everywhere!) examples of primary metaphors: 
positive emotions are warm (an experience that is established in very early childhood), something important is large, more is up/less is down, organization is physical structure, happy is up/depressed is down, states are locations, causes are forces, causality is forced movement, goals are destinations, time is movement, control is up, help is support, difficulties are burdens and categories are containers (p. 179). Each one of these is bodily anchored in a relatively straightforward way, but Johnson also examines a number of conceptual metaphors that are "further away" from immediate bodily experiences, but he still believes they can be traced back to the body, for example, within mathematical cognition. These are not primary metaphors, but conceptual metaphors and I would recommend the reader consult Johnson's works to gain insight into them.

If we wish to apply this metaphor theory critically, for example, to criticize existing metaphors, then the goal cannot be to rid our language of metaphors in order to arrive at an original, non-metaphoric understanding. Without metaphors we would not be able to understand anything abstract whatsoever. In that sense, metaphors are cognitive and indispensable; they enable us to learn a great deal of complex aspects of the world. They are, in a manner of speaking (metaphorically!), a sort of fuel for the mind, which makes it "run" when we have to deal with abstract phenomena. Of course, it may be legitimate to criticize certain metaphors for being fruitless and we may want to insert other more fruitful metaphors instead. I will not elaborate on this line of thinking here, but it might be interesting to consider such an exercise with regard to seeing and grasping metaphors for understanding. Johnson himself is inspired by pragmatist philosophy, which is not so concerned with whether something is true. We do not ask "is the sight metaphor true?" Instead, pragmatism is interested in which ways of acting and experiencing are made possible if we base our actions on a particular idea. So instead we ask: "which actions are enabled by the sight metaphor - and which are excluded?" Whether one metaphor is better than another will depend on what effects it has (from a pragmatic perspective) on constructing a social practice. So, the question is not about which educational metaphor is universally true, but about what opportunities for human learning, development and experience the two metaphors make possible.

All in all, the theory about our cognition being anchored in body-based metaphors provides a promising approach to understanding the body's role in the creation of meaning, including the creation of meaning in abstract concepts and theories, and thus for understanding the mind itself. In a broad sense, the mind is the part of us that has to do with meaning, which is why it is important to understand the body's (or body-mind's) role in the creation of meaning. Dewey believed that the mind is the ability to respond to contexts of meaning: "The difference between an adjustment to a physical stimulus and a mental act is that the latter involves response to a thing in its meaning; the former does not" (Dewey, 1916, p. 29). If we respond to the world's stimuli in a purely causal way, such as when a doctor tests our reflexes by tapping our knee, then we are not dealing with a mental process. We are not dealing with a mental process until we respond to 
the meaning that something has, and the reaction in this case is normative, since there are norms for correct ways of reacting to meaningful events. We can conclude from Johnson's metaphor theory that the body is a necessary condition for the existence of the mind because it forms the basis for experience and creation of meaning.

\section{The body's habits as background}

Understanding the body's role in the creation of meaningful experience through metaphors is essential for understanding the human mind, but the body has other, and in a sense even more basic, functions in our mental lives. In a very deep sense, our bodies form the background for our conscious experience; it takes on this role because it is the seat of our habits. In this sense, the body is the background for the mind. What do I mean by "background"? When we look at philosophy in the twentieth century, we can interpret many of the most important philosophical discussions as having to do with the background. A technical way of putting it is that the background consists of the non-intentional conditions for our intentionality. This means that what we think, feel or want can be in the foreground only because there is a background. In Chapter 2 we saw an example, inspired by Wittgenstein, where a "picture" (e.g. in the form of an imagined mental representation) that unmistakably depicts a heavy person, does not itself indicate whether it represents a pregnant woman, a fat man or something else entirely. The picture is technically speaking intentional because it represents something; it refers to something other than itself. But it is not self-interpreting. It has to be interpreted by a person in order to mean something specific and the concept of "background" should point to the fact that it can be interpreted in a particular way, and thus mean something specific, only against the background of something else that is not itself intentional and which is part of the interpretation.

Another example may help us to understand what the background is (inspired by Searle, 1992): if two people are standing in front of a locked door, and one says "Give me the key" there is, in principle, an infinite number of ways that this utterance can be understood. In practical terms the utterance is almost always understood the same way as a foreground phenomenon: the person fishes in his pocket for the key and then passes it to the other person with his hand. This is a very simple process. But it is possible that the person could have swallowed the key and, using his stomach and oesophagus, he could have regurgitated the key and spat it at the other. Needless to say, this is an inappropriate way to give someone a key. Or the person could have gone to the post office to send the key to the other person's address. Or the person being spoken to could have handed over a key different from the one that works in this door. It was not explicitly stated that the person wanted the key to this door. For that matter, it was not explicit what was meant by a "key" in the first place. It might have been a car key, an answer key, a piano key or something else entirely. One could keep going like this: The possible interpretations are endless. The concept of the background explains why, in real 
life, we rarely misunderstand each other when we communicate and interact with one another in everyday life. The reason is that we share a common, non-thematic background through common habits. The likelihood of misunderstandings is obviously greater when people from different cultures interact, i.e. if they do not have the same background, but given that we are all human beings and share a bodily being-in-the-world to a great extent, there will be a certain degree of common background.

Searle, who is one of the most important theorists with regard to background, states that the background is not a system of rules (which computer functionalists generally believe) because rules can be applied only relative to a set of background capacities (Searle, 1992, p. 193). This means that the rule to "look in your pocket for the key-shaped object when a person asks for this object" itself has a certain meaning only in terms of a certain background. We can articulate more and more what we mean by the individual words, but any attempt to make this more explicit will unlock an infinite number of new possibilities for interpretation. We never reach something uninterpreted or given. Meaning is contextual and holistic in this sense. The fact that meaning is holistic means that nothing has meaning in itself, but only within a larger whole or a network of meanings. In order to have a single belief (e.g. that Mount Everest is 8,848 metres high), I need a network of other beliefs (about what a mountain is, what the metric system is, what height is, etc.) and such a network has meaning only based on a background, since none of the elements in the network are self-interpreting (p. 176). The background fundamentally consists of bodily anchored social practices, of abilities to act in social contexts of meaning (Dreyfus, 1991).

\section{The mind as habits}

Another, more psychological concept for the background is the concept of habit (Brinkmann, 2013). Dewey believed that the concept of habit ought to be fundamental to an understanding of the mind, but the concept has unfortunately fallen out of favour since then, especially because of the dominant position that the computer functionalists hold. The concept is interesting in that it mediates between socio-cultural and bodily aspects (Dewey, 1922). Habits are both at the same time: culture and body. Dewey stressed that it is through habits that we have practical knowledge of the world, or know-how (p. 177). Habitual know-how is a condition of our more reflective and articulable knowledge of how things are constituted. Things appear in a certain way in the foreground only because we have certain background habits with respect to interacting with them. The radiologist who has habitually trained his skills at the hospital is able to see things in X-rays, i.e. in the "foreground", which others fail to see. Habits are inscribed in the way our body unreflectively works, but at the same time they reflect the socio-cultural conditions of a society. Moreover, habits are more fundamental than both subject and object. We should not accept any strict division between organism and environment. We must begin by looking at the overall life process, 
and the concept of habit is a productive point of entry for this process. Habits therefore do not entail just a "mental" dimension, but an "external" and material one as well, for instance in the form of tools and artefacts. Dewey states that: "Habits are arts, involving skill of sensory and motor organs, cunning or craft, and objective materials" (p. 15). Anyone who is a master stoneworker has specialized in certain habits that allow him to interact productively with his material, and the material and tools are just as much a part of his habits - and thus part of the person's psychological constitution - as that which is "inside" him, e.g. brain and muscles.

It is through habits that we exist in the world, and they allow us to feel at home in the world as well. It is because of our habits that we "in-habit" the world (Dewey, 1934). To inhabit the world through habitual activity is largely a nonreflective and bodily process. Dewey stresses that our habitual background knowledge "lives in the muscles, not in consciousness" (Dewey, 1922, p. 177). Our knowledge of how to ride a bicycle is not conscious knowledge, but physically based, practical knowledge. It thus becomes clear that habits are not something separate from us, not some external element that we can take up in order to master the world. We do not have a mind with intentions prior to, or independent of, our habitual behaviour. It is more correct to say that "we are habit", "they constitute the self" (p. 25). The mind is constituted by habits, and habits have a social origin, which leads us to the conclusion that the mind is social. This points us in the direction of the next chapter, which is about the mind's sociality.

The basic point of this explanation about the concept of habit is to emphasize that, as psychological beings, we can reflect consciously on something specific only because so much else is habitual. We can put something in the foreground only because so much else is in the background and this background must be fundamentally understood as constituted by our bodily habits. Maybe we can also find seeds for a reinterpretation of the unconscious here, which played such a big role in psychology with Freud's psychoanalysis in the past? Perhaps we can say that the unconscious is not so much a mental world in its own right - a world that the conscious mind is prevented from gaining insight into directly - but instead that it is made up of habitual patterns that our "body-mind" uses to guide its functions, and which are already established in early childhood through our interactions with caregivers. In short, the unconscious is perhaps what I referred to as the background above. Part of this background works metaphorically, as Johnson would say, to create meaning in our experience, and part is even more implicit in our unreflected bodily behaviour.

\section{Development of the body-mind}

Developmental psychology deals with how the abilities and dispositions of the mind develop throughout life, and although this book does not primarily aim to take a developmental perspective, it is nevertheless appropriate to outline how the body-mind develops in the earliest years of our lives. We are born as physical 
beings with concrete experiences of hot and cold, satiety and hunger, and only later do our cognitive abilities develop to understand abstract phenomena. It is clear that we must develop a language with all of language's relevant concepts in order to understand phenomena such as democracy and the climate crisis, and it is fascinating to think that our ability for abstract understanding, thinking and reasoning is associated with fundamental body-mind processes. Modern developmental psychology has been oriented towards at least two key things: the first is that young children can do much more, and at a much younger age than previously thought, and the second is that young children are social from birth. The British psychoanalyst Donald Winnicott (1896-1971) said that there is no such thing as a baby - there is only a dyad, a unit consisting of an infant and its primary caregiver. The dyad - not the individual - should be the smallest unit of developmental psychology. These two main developmental psychological theses are related. Young children can do so many things (almost) from the beginning because they are not alone, but are included in ongoing social, dyadic processes. For example, researchers have been able to demonstrate that newborn infants - as early as 42 minutes after delivery - are able to imitate adult facial expressions (Meltzoff \& Moore, 1997). They can, for example, imitate adults sticking out their tongues, and they do so with a certain delay, which shows that it is not pure reflex. However, infants cannot imitate everything. They imitate human facial expressions, but not the movements of inanimate objects, and this means that even very small infants sense that other people are subjects to be imitated and not merely objects. More recent infant research has also demonstrated that even very young children possess a basic capacity for empathy.

The capacity for empathy is an ability to understand others as others - in other words, it is a capacity for intersubjectivity. As psychological beings we are necessarily born in intersubjectivity (Trevarthen, 1993). A classic study using television screens demonstrated intersubjectivity in young children purely experimentally (Murray \& Trevarthen, 1985). The researchers allowed infants between six and eight weeks old to interact with their mothers via television screens. Already at this age, mothers and children communicate intersubjectively in a way that can be described as a dance or as an interplay of facial expressions and sounds. This is a dyadic unit where the body-mind unfolds in a primitive but fascinating way. These "dance steps" are presumably biologically based and are crucial to child development. The children and the mothers could easily communicate with each other via the screens, as long as it was happening in "real time", but when the researchers manipulated the transmission and delayed it by a half minute, the infants responded by looking away from the screen and showing signs of discomfort. The rhythm of the intersubjective dance was disrupted and the infants could not get the intersubjective response they expected from their mothers. This and other similar experiments show that it is not just a speculative philosophical assumption that we are born in intersubjectivity, as social beings, but that it is something we can demonstrate empirically. 
During the first months of children's lives, they possess a form of primary intersubjectivity. This means that child and mother both share in the communication and expect certain responses from each other, but they do not yet share in anything outside of the communication itself. Around the age of nine months, the communication starts to change gears towards secondary intersubjectivity. The parent and child start to share in something else during communication. They can engage in paying joint attention to objects and events. At this stage mothers are now able to start pointing to things, and children will look at the things rather than just looking at their mother's finger; children also begin to be able to follow the direction of their mother's gaze. Children will also be able to draw adults' attention to things in the world. Secondary intersubjectivity is undoubtedly a necessary precondition for people to be able to learn languages, since essential parts of language work through pointing to referents (Krøjgaard, 2007). Meaning and significance - including that of the language - are rooted in the fact that we can use one thing to represent another. We can use sounds and signs to identify and refer to specific objects and events, and this is an ability that develops out of basic physical and psychological interactions during the child's first year. Most children will also say their first words when they are around one year old. Our capacity for language and abstract thinking is not based on "cold", mechanical cognition, but is founded on "warm", empathetic and intersubjective interactions with caregivers. Body-mind feelings are the foundation for language and cognition. From nine months of age on, children begin to understand references to things outside of the primary communication process, and this is also when they begin to be introduced into the cultural and social community of contexts of meaning. In the next chapter, I will continue with a description of the part of the mind's development related to this stage.

\section{Conclusions}

For now we can restate the lived body's importance for the mind. In Chapter 2, we saw that the brain is a necessary, but not a sufficient, condition for the existence of mental life; in this chapter, we encountered a similar argument with regard to the body. It was emphasized that the mind is so closely linked to the body that it may be appropriate to talk about the "body-mind", and we have seen examples of the body's role in the creation of meaning, which is a crucial feature of the mind. The body should not be understood as an object in this context, but as a living and lived body. So being a bodily entity, being a moving, physically acting being, is a necessary condition for the mind - but is it also a sufficient condition? No, because as this chapter has also shown, the normativity that the body (or "bodymind") follows in life, and which is defining for mental life, is not understandable from looking at the body alone. Furthermore, we have to keep in mind the basic lesson of personism: that psychological terms make sense only when applied to persons. Thus, just as we cannot meaningfully say that the brain thinks, so we also cannot say that the body thinks. But what we can say now - after having 
reviewed significant ideas about the brain and the body - is that persons can think only because they are cerebral and embodied creatures. The brain and the body are indispensable mediators in the psychological processes in persons' lives. If we want to understand the mind as a range of rational abilities and dispositions, we therefore have to involve not just the brain but also the body. But we also need to involve the community and sociality, which establish norms for how these abilities are formed and how they play out in social life. This will be done in the next chapter, where society, or the social community, takes centre stage as a basic condition for the human mind. 


\section{Chapter 4}

\section{The mind and society}

Man is a social being. Animals other than humans live in groups or flocks, some animals even have a division of labour (e.g. ants and bees), and some species can hand down certain behaviours that are not coded in their genetic material over a span of generations. For example, researchers have observed that some monkeys can learn to wash potatoes from their elders (Kawamura, 1959). This is one definition of culture: the ability to pass on learned patterns of behaviour. But no animals besides humans are able to negotiate the norms that apply to their culture or community. No other animal can reflectively consider whether the current practices in a group are the most reasonable. For some non-human animals, group dynamics are such that when the top of the hierarchy becomes too weak to be alpha male, for example, a new alpha male will take his place, and in that sense there is a change in the distribution of power among the individuals in the group. But what does not happen is a fundamental structural change in the power relationships. Monkeys cannot consider whether the alpha-male system is fair. And democracy will not spontaneously arise within the group of monkeys. No other animals besides humans can consciously change the norms that apply to social life. No other animals besides humans are therefore political in the proper sense. The human being is a political animal - a zoon politikon (Aristotle, 1978). This obviously does not mean that all norms are constantly under discussion in human society, although you almost get that impression in the post-modern era, but it does mean that, in principle, it is possible to negotiate almost every aspect of our social lives, as long as we do not negotiate all of them at the same time.

One word for man's ability to take part in a world of norms, traditions, institutions and practices, which we constantly reproduce and can negotiate to some extent, is spirit. When a philosopher such as G.W.F. Hegel (1770-1831) used the term spirit, he did not mean something ethereal or ghostly. Spirit is what the humanities have as a subject, i.e. the man-made, social and historical world in its meaningfulness. This is where man lives and develops as a psychological creature. Søren Kierkegaard (1813-1855) asserted in his definition of the self, that the spirit is the self, as a relation that relates itself to itself. We are all spiritual beings in this sense, when we not only stand in an immediate relationship to the world, but can relate to how we relate to the world. We can do this because we can avail ourselves 
of language and other meaningful signs. Through the use of signs, we can establish a distanced or mediated relationship to ongoing processes in the world, which is often necessary when, for example, we need to make a choice (Valsiner, 2007). We create distance from here-and-now situations, and can thus consider future actions, when we use language to imagine what is not - or is not yet - the case. Spirit, culture and language are therefore inextricably linked.

The world of the spirit is not static or unchanging, but is historic, changing and normative. Hegel and his successors, especially Karl Marx (1818-1883), were intrigued by the duality that exists in the fact that people both live in history, and thus are a product of their historical circumstances, but at the same time they live in history, and thus themselves constantly produce the circumstances that shape them (see Lave, 1993). Man's historical life is a social life. We can have our own individual life history only because we take part in the "spiritual" world's larger history through which we acquire a language that we can use to think about our own existence, and a set of norms for how we should live our lives. Human selfknowledge is social and historical - or as the hermeneutical philosopher HansGeorg Gadamer (1900-2002) said:

$[\mathrm{H}]$ istory does not belong to us; we belong to it. Long before we understand ourselves through the process of self-examination, we understand ourselves in a self-evident way in the family, society, and state in which we live. The focus of subjectivity is a distorting mirror. The self-awareness of the individual is only a flickering in the closed circuits of historical life.

(Gadamer, 1960, pp. 276-277)

Psychologists have tended to direct their attention to the individual mind without giving much thought to historical life, society and the state. But if Gadamer, Hegel and Marx are right, then this focus on subjectivity is in itself "a distorting mirror". To get a picture of the human mind that is not distorted, we need to look at the mind as a social product. According to the OED, society comes from Latin societās meaning the condition of being associated for a common purpose, partnership, fellowship, communion or joint pursuit. The fact that the mind, in its complex manifestation, is dependent on people coming together in joint pursuits is the subject of this chapter. In the previous two chapters we saw that the mind can exist only because we have a brain and a body, but we must not forget that society is an equally necessary condition for the existence of a fully human mind. It is one of the mind's mediators.

I begin by outlining the basic assumptions behind the forms of current thinking about the mind that most clearly present the mind as a societal entity, often in continuation of Hegel and Marx. This concerns socio-cultural psychology in a broad sense. In this tradition, mental life is primarily linked to social activities. The mind is a range of abilities and dispositions and not a thing. The description of socio-cultural psychology will subsequently be combined with an explanation of the normative dimensions of human sociality, which are centred on the concept 
of social practices. Finally, I will briefly touch on how different cultures find expression through various forms of mental life, and I will give a brief analysis of a central arena for mental development in most societies: the family.

In short, the chapter's mission is to continue to expand the boundaries of the mind begun in the preceding chapters: we cannot say that the skull or the body's skin forms the boundary line for the mind; we must also involve the society in which mental life takes place. However, we need to be careful with the spatial metaphors here. The brain is inside the body in a completely literal way, but the body is not "inside" society in quite the same way. Thinking metaphorically about society as a container that holds individuals is not a very productive approach, since it invites us to analytically separate the individual from society, culture and community. It invites us to reify the mind as a thing and to think about society or the social realm as a kind of material (Latour, 2005, has attacked this substantial idea about the social). Neither the mind nor society is a "thing", and the individual is not "in" society, like an apple is in a fruit bowl or the Eiffel Tower is in Paris. We can take an apple out of the bowl and still have an apple, but we cannot in the same way abstract the mind from societal processes and still have a mind. We are not in society like apples in a bowl, but more like events in history. If we abstract a particular event out of the historical circumstances surrounding it - for example, the fall of the Berlin Wall - then the event, as such, ceases to exist because we cannot understand the fall of the Wall without understanding the historical background and the importance of the political ideologies involved. The interesting thing about the fall of the Wall was obviously not the physical collapse of the structure (a nomic process), but the meaning that the physical collapse had (an intentional and normative process). This brings us to the societal world of meaning and normativity, which is crucial for the existence of human mental life.

Just as the mind is not a thing, neither is society. We cannot take a walk and observe society as an object. Society is an abstract name for certain relationships and processes among people, which are sustained by social practices, institutions and technologies. The concept of society did not come into widespread use until the nineteenth century and since then people have regarded it as something akin to a secular deity with its own wants and needs (Valsiner, 2007, p. 75). For example, we can tell people that they need to do something "for the sake of society", whereas in the past we would have said they need to do it "for God's sake". Politics are conducted in large part by creating and debating certain conceptions about society.

\section{Socio-cultural psychology}

A line of work in socio-cultural psychology has developed in continuation of Hegel and Marx, which is based on the fact that man is a social being. Parts of socio-cultural psychology are also inspired by Wittgenstein and his life-form concept. Michael Cole, who is a major contemporary researcher in socio-cultural psychology, mentions three basic principles for this type of psychology (Cole, 
1996). The first is that the development of the mind takes place through artefacts. Artefacts are anything created through crafts, arts or other forms of processing of materials by human hands. This means that the mental processes in their present form arose when people began to reshape material objects into artefacts and tools in order to regulate their interaction with each other and the rest of nature. An evolutionary leap occurred at the point when people started using tools and thus acquired culture. The second principle is that there is (therefore) a basic assumption that human life is the result of historical development within a cultural context. Cole defines culture as "the entire pool of artefacts accumulated by the social group in the course of its historical experience" (p. 110). In this way, culture is the medium for development of the mind. A person without culture is a myth, because even the earliest interactions between infant and caregiver are imbued with the given cultural context. The third basic principle Cole lists is that scientific analysis of the mind must build on the fact that human beings are engaged in practical activities of daily life. We are primarily participants in various practices rather than spectators who observe the world from a distance. As established in the previous chapter, we are first and foremost beings who conduct ourselves actively and whose relationship with the world is one of use. And the things we "use" as mental beings are primarily artefacts and language.

If Descartes, Hume and Kant are background figures for the problematic cognitivist and mindist form of psychology that was discussed in Chapter 2, then other philosophers have inspired socio-cultural psychology. As mentioned, these were specifically Hegel and Marx, but Darwin's naturalism also plays a role, particularly because of its emphasis on the changing nature of the world. In addition, Aristotle's emphasis on the socio-cultural context of human life can be found in the views of socio-cultural scientists. However, the most significant psychologist within this tradition is probably the Russian Lev Vygotsky (1896-1934). He founded the specific tradition that is referred to as cultural-historical psychology. Vygotsky was involved in investigating mental functions such as thinking, memory, will and language, not as static phenomena that are permanently established, but as processes in development. He distinguished between natural or lower mental functions on the one hand and higher mental functions on the other (Vygotsky, 1978). According to Vygotsky, the former are developed dependent on biological maturation and include, for instance, our basic sensory abilities, while the latter are cultural and developed in individuals when they participate in social practices.

The development of the higher mental functions in individuals is a result of the individual's mastery of certain cultural tools. Tools should be understood in a broad sense here, including language, for example. A simple example of a tool that is linked to the development of a mental function could be knots on a string, which some pre-Columbian cultures in South America used as a tool to aid memory (Chaiklin, 2007, p. 277). Use of this tool was rooted in specific needs and challenges within the given society to keep track of goods, and therefore the practice of using it to assist memory developed. Once a social practice in which this artefact is used is in place, new generations will learn how to use the tool by 
participating in the practice in such a way that the practice is reproduced. Social and historical factors thus go in advance of the individual. In the next chapter we will focus in more detail on the significance of the world of things and artefacts for the mind, in particular on the significance of tools and technologies. From this perspective, higher mental functions - and thus the specifically human mind - are a product of social history (p. 276). Briefly put, the mind is social.

There is also a developmental approach built into this perspective since the individual psychological level must be understood as an internalization of socially and historically determined patterns of interaction. The individual's mind reflects sociality, so to say. We develop mentally when we increasingly come to master the social practices on which a given society is dependent (agriculture, medicine, construction and many others). Bearing the concept of habits from the previous chapter in mind, we can say that society, with its social and cultural practices, precedes individual habits. Vygotsky famously argued that every mental function appears twice during children's development: first socially, among people, and then individually, "inside" the child (Vygotsky, 1978, p. 57). For Vygotsky, the mind is thus a function of the social world. A well-known example of this is his analysis of the development of the ability to point. Adults have no problem identifying objects in their surroundings, normally using their index finger. As we saw in the previous chapter, such intentional movements are only possible because there is a background that is not thematized in the action itself; for example, the observer is expected to look in the direction of the index finger and not in the direction of the elbow, which is just one of many background conditions for pointing. The ability to understand pointing references begins at around nine months of age with the development of secondary intersubjectivity, where the communicating parties can share something outside of the communication process itself. I made reference to this earlier, but how do we understand in greater detail the child's beginning mastery of the activities that include ostensive or indexical references such as the act of pointing itself?

Vygotsky took an interest in how such intentional behavioural patterns are developed. He observed that when young children make this gesture, it is initially an (unsuccessful) attempt to grasp an object (Vygotsky, 1978, p. 56). Children simply reach for objects that they find appealing for one reason or another. The predisposition to make such grasping movements is an innate part of the child's elementary mental functions that mature in the child's early life. But when the child's caregivers become aware of the failed grasping movement, they will typically react. They will often bring the child what it is reaching for, and Vygotsky's point is that the movement is thus slowly transformed from being a grasping movement to one of pointing. The child realizes that it can use this movement not only to grasp things, but as an intersubjective sign that is understood by others and often results in the child getting what it wants. Pointing is thus established as an action - as a meaningful movement - in a process Vygotsky called internalization. The movement goes from being reflexive to being intentional and normative.

Meaning thus becomes an attribute of the child's behaviour when the behaviour forms part of a larger social context with other people and objects - in other words, 
when it forms part of a social practice. Dewey, whose pragmatic psychology can also be characterized as socio-cultural and reminiscent of Vygotsky's in several respects, illustrates this with an example that looks at Vygotsky's pointing scenario from a slightly different angle:

$A$ requests $B$ to bring him something, to which $A$ points, say a flower. There is an original mechanism by which $B$ may react to $A$ 's movement in pointing. But natively such a reaction is to the movement, not to the pointing, not to the object pointed out. But $B$ learns that the movement is a pointing; he responds to it not in itself but as an index of something else.

(Dewey, 1925, p. 178)

Vygotsky described the pointing scenario from the child's perspective, while Dewey sees it from the other angle, from the perspective of the one observing the pointing. The argument is nevertheless the same: humans have a number of instinctive ways of reacting, for example, reaching for things and reacting to the movements of others. Such background ways of reacting are elementary as mental functions and in order to understand how the process is developed in the direction of higher mental functions, we have to take an interest in the meaning or significance that movements can have. A pointing movement in itself is not meaningful, i.e. as a purely physical event. It does not become "pointing" until the movement is regarded within the context of a larger practice across contexts and time, where people can point out objects together. The movement also becomes part of a system of normative contexts in this respect. Not all objects, for instance, are legitimate to point to. The child learns later, at least in our culture, that it is inappropriate to point at strangers on the bus; in other cultures different norms may apply. The pointing example may seem banal, but it illustrates the fact that even such a simple gesture as pointing is part of a complex social choreography that is based on expectations and norms that are often not explicit, but which instead form part of the background. And when a person gradually learns to understand the practices associated with pointing, as well as a host of other social practices in his or her culture of course, then the person's mind itself (i.e. the capacity to respond to normative contexts of meaning) develops. Psychological development is primarily about being led into society's system of contexts of meaning; it is about becoming a full-fledged participant in social practices. A key Vygotskian insight is furthermore that this kind of development happens in what he called the zone of proximal development, where more capable individuals, typically adults, do something together with the less capable individuals, e.g. children, in a way that scaffolds the activity. Gradually, the novices (or apprentices) become able to perform the actions without the support of experts (or masters). A precondition for this is that masters treat the novices as slightly more capable than they actually are in order for them to develop (hence: the zone of proximal development). For example, adults must thus interpret the grasping gesture of the child as an act of pointing, before it really is pointing, in order for the gesture to gradually become 
an act of pointing. More generally, this is how we become intentional and agentic persons: By being treated as such, before we actually are such persons, in order for us eventually to become persons. This is a most fascinating aspect of psychological development.

Vygotsky's work was motivated by one main problem in particular: how to understand the origins of humans' free actions and conscious behaviour (Chaiklin, 2007, p. 274). As we have already seen, the answer is given based on a theory that people learn to use tools and signs in social contexts that give them a mediated relationship with themselves. Thanks to language and other tools, we can establish a "distance" to ourselves - our immediate impulses and preferences where we can consider both the past and the future reflexively. An illustrative example of this from Vygotsky is Buridan's ass, which is taken from amongst the paradoxes of medieval philosophy (Vygotsky, 1997). A hungry ass is precisely midway between two equally appetizing piles of hay of the same size. What does the ass do? According to the classic paradox, it does not do anything because it is confronted with two equally strong stimuli, so it cannot make a choice and ends up starving to death. What would a person do in the same situation? According to Vygotsky, humans are capable of choosing and acting because, in contrast to the ass, we do not just react to stimuli directly, or in this case not react because two stimuli are equally strong. People themselves contribute to structuring the stimuli that confront them because they can use historically developed tools. For example, a person in this predicament could flip a coin, because we have developed such a practice for getting us out of this type of situation. We can introduce stimuli in the form of signs and symbols for ourselves, and use them to make the choice. As a higher mental function, the will is thus mediated by tools, just like other higher mental functions such as memory or thinking. The mind's higher functions are developed via the acquisition of societal tools and signs, and we can gain control over our actions by using them (Chaiklin, 2007, p. 279).

An essential point is therefore that, in the socio-cultural tradition, society is not regarded as an impediment to the individual's free opportunities for development. Society is not something that limits the mind's functioning. On the contrary, the social practices of society are a necessary medium for a mature mental life in humans to exist at all. Kant tells a story in his first Critique from 1781 about a flying dove that, while soaring freely through the air, fantasizes about being able to fly much faster in a vacuum since there would not be any wind resistance. The point of the story is that the dove would not be able to fly in a vacuum, because the wind resistance is precisely what keeps the dove in the air. And it is very much the same with the society in which human life unfolds: the social conditions and norms for right and wrong are like a kind of mental wind resistance, enabling us to have a mind in the first place. Society is not a limitation of individual freedom, but a prerequisite for it.

After Vygotsky's death in 1934, cultural-historical psychology continued to develop in various directions, but especially in the direction of what is called activity theory (Mammen \& Mironenko, 2015). An important figure in this 
development is A. N. Leontiev (1904-1979), who developed a systematic analysis of the organization of human activity and practice to a greater extent than Vygotsky himself (the reference is to a Danish translation, and his name is here spelled differently: Leontjev, 2002). The term "activity" here refers to specific social "activities" (or social practices), often with a division of labour, such as education or production. According to Leontiev's analysis, an activity contains a particular "motive", which can be said to be the purpose of the activity, for example, educating people to carry out societal tasks (Chaiklin, 2007, p. 290). The motive can be achieved through many different kinds of actions, and actions can in turn be carried out through a number of different operations. A basic fact of action theory is that even the simplest actions can be physically carried out in an infinity of ways (for example, a door can be opened with your hand, foot, mouth or a stick) and Leontiev's term for these action-oriented movements is operations. Activities consist of actions that in turn consist of operations, and this perspective allows us to link a general, societal level of analysis with an individual, psychological one. We can link social practices with actions. Below we will take a closer look at the role that the concept of social practices can play in relation to a socio-cultural understanding of the mind.

\section{The mind and social practices}

Social reality is historically changeable, open to multiple interpretations, and in contrast to physical reality it is negotiable. We can take a vote, in a meaningful way, on whether we should have car-free Sundays in Denmark, but not on whether we should repeal the law of gravity on the first Saturday of every month. Despite its fundamental changeability, however, social reality still exhibits a relative stability and order. Unless we are mentally ill, we do not we get up in the morning and encounter a completely fragmented and confused social world, but rather a quite coherent and comprehensible reality. The order and regularity that events in the physical world exhibit can be explained nomically with reference to universal laws as described by great scientists such as Isaac Newton and Albert Einstein (1879-1955). The question here is whether the relative order and regularity that events in the social world exhibit can be described by universal laws in the same way. Many socio-cultural scientists answer this question in the negative. The socio-cultural world is not ordered causally, but normatively, and norms can be said to be embedded in the social practices of society.

A simple example will serve to illustrate the difference between nomic and normative reality. There is a fundamental difference between (1) a situation in which an automatic door opens when a photocell's light beam is interrupted, and (2) a situation in which a person opens a door for another person. The first situation is explained comprehensively when we know the mechanics of the photocell and the door, and this mechanism can be reasonably assumed to operate according to the same universal laws, whether the automatic door is in a supermarket in Sheffield or a bank in Beijing. In the second situation, however, just knowing 
the "mechanics" (e.g. the human brain and locomotor system) is not enough to understand what is going on. Even though the "mechanics" might be universal physiology is essentially identical in Sheffield and Beijing - the same is not necessarily true for the act itself. To understand why someone opens a door for someone else (say, a man opening the door for a woman), we have to know this person's perception of the situation and the set norms and conventions of the given location. The man's action might be a kind-hearted attempt to help the woman, or it might be an attempt to be the last one to enter an uncomfortable encounter and to hide himself at the woman's expense. Knowledge of causal laws does not help us to clarify whether the action is the one or the other. Only knowledge of the person's intentions and the greater cultural context helps us in this regard. In many cultures the man's action could be understood as an expression of courtesy, based on a perspective of the sexes where the man is perceived as the active protector and the woman is perceived as passive and weak. In other cultures where feminism has taken hold, the action could be perceived as condescending to the woman because it unduly portrays her as being someone who needs help and protection simply because she is a member of the female sex.

In this way, human mental life can be comprehended only if we are familiar with both people's intentions and the applicable local norms. We need to know what people are trying to achieve (intentions), and we need to know what is considered becoming and unbecoming, right and wrong (norms and conventions), in order to understand what is happening in a given social episode. We can take note of two things in this example: first, we can point out an action in the flow of events only because we can place the action within a general social practice. Every action as an action presupposes the existence of a practice for performing the action. I can promise my family that I will do the dishes only because there is a practice we call "making promises" and another we call "washing up". This utterance would be meaningless if this were not the case. If there was only a single person who had ever made a promise and if this had only happened once in the course of history, then we would not be able to understand what a promise was. One of Wittgenstein's key points is that an action can make sense only as part of a publicly observable practice of performing that action over a span of time. According to this perspective on actions, there is no external view, distanced from human actions, from which existence can be understood. To understand human existence is to interpret an interpretation - which means, to interpret how the actors themselves interpret their world - and this can be done only "from inside" the interpretations themselves - by being familiar with the actors' practices - and not "from outside", for example, by using the objectifying methods of the natural sciences. We need first and foremost to know the local normativity rather than the causal mechanisms.

In the words of cultural psychologist Richard Shweder, we need to know "the intentional world" (Shweder, 1990a). According to Shweder, the intentional world is the subject of the cultural-psychological exploration of the mind. He defines cultural psychology as "the study of the way cultural traditions and social 
practices regulate, express, transform and permute the human mind, resulting less in psychic unity for humankind than in ethnic divergences in mind, self, emotion" (p. 1). Shweder believes that the mind is a product of social practices. Our sociocultural environment is perceived as an intentional world. The Berlin Wall, as discussed above, is only of importance because it is part of the intentional world. If, by some cosmic coincidence, a physically identical wall had been formed by the wind and weather in the Kalahari Desert, it would obviously be quite a sensation when it was discovered, but the "wall" would not really have any historical significance other than as a geological phenomenon. In a way it would not even really be a wall at all, since a wall is a structure that is created by people under certain historical circumstances in order to separate something from something else - i.e. it is based on certain ideas and social practices.

The intentional world is organized in and by social practices. We could say that individual expressions of human mental life - thoughts, feelings, actions - all require a practice that gives the individual expression meaning. Such a practice is normative, since it is based on a difference between proper and improper. This was illustrated in Chapter 1 with anger as an example. Briefly put, mental life is ordered by societal norms, without which our forms of mental life could not exist, and these norms are realized in social practices.

But what are such practices more specifically? More recent social theory has taken a "practice turn" (Schatzki, 2001). In the middle of the twentieth century we saw a "linguistic turn" in the humanities and social sciences, but in recent years the concept of "practice" has proved productive in rethinking some of social theory's basic questions. Social scientists like Anthony Giddens and Pierre Bourdieu believe that the concept of practice will be able to dissolve the (inappropriate) dualisms between actor and structure as well as individual and society. Both are viewed as being derived from a more fundamental level of practices. In the previous chapter I attempted a similar exercise based on the concept of habit. Andreas Reckwitz has created an overview of the diversity of practice theories and concludes that a common characteristic is that practice theory does not put the social into mental qualities, discourse or interaction, but into the practices themselves (Reckwitz, 2002, p. 249). He defines a practice as "a routinized way in which bodies are moved, objects are handled, subjects are treated, things are described and the world is understood" (p. 250). This is at first glance a fine and inclusive definition, but it fails to mention an essential element of a practice: that is normative. Reckwitz emphasizes only that they are routinized, meaning that we have a practice when many people act in the same ways over time.

For the purposes of this book's approach to the mental as something normative, it is more productive to follow Rouse and define practices as normative accountability (Rouse, 2007). This means that the actors in a practice are accountable to the fundamental normativity that constitutes a practice. Regularity, i.e. the fact that people behave the same way, is not sufficient for us to have a practice. For example, most people open the door with their right hand, but this is not a practice 
in the strict sense because there is no normativity involved. We could say that this is a statistical normality, but in the normative sense it is a coincidence that one hand is used more often than the other. There is no reason for habitually opening the door with our right hand, but there is definitely a cause that has to do with common brain lateralization and the resulting left- or right-handedness. But people are not corrected if they use their left hand, and the concept of normativity is, as mentioned, closely linked to the possibility of making mistakes and being corrected.

If the presence of normative accountability is necessary for us to be able to talk about practices, then we need a theoretical definition that takes this into account: the Canadian philosopher Charles Taylor provides one when he defines a practice normatively as "any stable configuration of shared activity whose shape is defined by a certain pattern of dos and don'ts." (Taylor, 1989, p. 204). Activities that do not contain such a normative pattern are not practices. But agriculture, music, politics, medicine, education of children, etc. are cultural and social practices because, as activities, they are steeped in norms. And as mentioned, we can understand individual actions (e.g. a child getting money for good marks in school) only if we understand the practices that make the action meaningful (in this case, educational and monetary practices in a capitalist society). Individual actions are the figure; practices are the background. The mind, as a range of abilities of persons to perform actions, respond emotionally and solve problems, is dependent on practices with their inherent norms in order to exist. We can "perform" mentally only if there are practices in which to "perform" and relative to which we can be held accountable.

If a person's mind is therefore to be understood as being situated in a social and normative field of practices, and if psychological phenomena therefore are normative, then the sciences of the mind come in contact with moral philosophy. It can even be expressed more strongly: in many ways, psychology can be seen as a part of moral philosophy (Louch, 1966). Within moral philosophy we are interested in the reasoning behind actions, and not in what causes behaviour. We concern ourselves with whether a way of acting and living is legitimate in an ethical sense, and not with the causal factors that underlie people acting or living in certain ways. I have argued in favour of the fact that psychological phenomena can only be understood if viewed within a normative framework, where we distinguish between legitimate and illegitimate, appropriate and inappropriate, right and wrong. This does not mean that all psychological phenomena are good in a moral sense. People can act maliciously and irrationally, after all. But it means that all psychological phenomena can be judged morally. We can judge a person's actions, feelings and thoughts morally - and they therefore belong to the field of the mind - but we cannot judge their physiology in the same way. Although in recent years a (problematic) tendency for moral condemnation has arisen, for example, with respect to obesity, it is not the weight itself that is morally condemned, but rather the person's actions that resulted in obesity. The actions are psychologically relevant and subject to moral discussion. 


\section{The mind across cultures}

The foregoing observations suggest that there is a close relationship between morality and psychology. MacIntyre has expressed it as follows: psychologies "express and presuppose moralities" (MacIntyre, 1988, p. 77). Psychological phenomena are partly constituted by the structures of normative practices through which they are expressed: our ways of feeling, desiring, thinking, perceiving and acting only have meaning within a particular "set of norms of justification" (p. 76). Some things are legitimate targets of our desires (e.g. a second family car), which in the past would have been viewed as unnecessary gluttony, and perhaps in the future this will change again. In my opinion, it is important to realize that psychologies presuppose moral systems - but the reverse is also true. Psychologies also "secrete" moralities (Much \& Harré, 1994); they invite us into thinking in specific ways about right and wrong. And the various psychological understandings and explanations of actions that exist worldwide are also brought about by certain fundamental metaphysical notions. A culture's psychological discourse reflects local metaphysics, i.e. a fundamental conception of people and the social order. Are people, for example, like atoms in social life (a widespread "Western" conception)? Or conversely, are people relational entities that are defined by their relationships to others (a widespread "Eastern" conception)?"

Various psychologies arise from various metaphysics, and various moralities emerge from those in turn. It is obvious that in the first-mentioned view (the "Western" one), the concept of rights plays an important role in the culture's morality. People as societal atoms have inviolable rights that take precedence over the considerations of communities. Communities become instrumental in terms of optimizing the lives of individuals and they cannot be seen as ends in themselves. In our part of the world we can hardly understand that it is possible to think differently, because the notions that underlie this thinking are so fundamental and pervasive in our lives. Conversely, if we take the "Eastern" view, the local prescriptive morality will typically be centred on the responsibilities that people have towards the community to which they owe their existence. All metaphysics and every psychology are teleological in this sense, meaning that they implicitly (or explicitly) say something about what should be (Much \& Harré, 1994, p. 293).

Cultural psychologists have conducted very thorough analyses of the relationships between moralities, metaphysics and psychologies. An example is an analysis of how the Christian view of the person on the one hand and Madhyamika Buddhism's view on the other give rise to different moral systems (Much \& Harré, 1994). In Christian cultures, morality is about mastering the lust of the flesh, because they have a metaphysical and psychological notion of a dualism between the pure soul and the impure body. The Buddhist view of morality, however, demands that we cultivate our cognitive processes, because it has a metaphysical and psychological notion that it is not the body, but certain thought processes, which deceive us into having a distorted view of the cosmos, thus preventing us from being enlightened. The conceptual worlds of entire cultures are connected 
to their basic psychological assumptions and associated social practices. Christian and Buddhist cultures, for example, have different practices with respect to exercising impulse control and mastering the lust of the flesh, respectively, which is essential in Christian cultures, and with respect to gaining insight into the illusory status of the ego, which plays a major role in Buddhism. Despite the widespread secularization of the Western world, I would argue that the basic Christian understanding of the person and the social order, and also the dualism between the spiritual and the corporeal, continue to structure many of our basic conceptions today. Not least of these are our conceptions about what the mind is, i.e. an inner "thing", as we saw in Chapter 1. Descartes could probably not have developed his dualistic philosophy in a non-Christian culture, and of course this dualism still sets the stage for our discussions about the mind.

If there is such an inner relation between psychology and morality, then we can either focus on how psychological conceptions arise from a culture's moral systems, or conversely on how a moral system arises from a culture's fundamental metaphysical and psychological concepts. When we deal with interpretive, cultural-psychological studies, it is not possible to determine in advance whether it will be most productive to keep the one or the other factor constant. What we can conclude is that cross-cultural studies almost always find that psychologies and moral systems vary together. Different cultures have very different conceptions of what the mind is, and they have correspondingly different conceptions of what morality is (although there might also be universally common ground between different cultures; see Shweder, 1990b). Theories about the mind are thus always moral and political as well - and vice versa.

In recent years, both cultural psychology and what is called cross-cultural psychology have been growing. The latter type of research compares mental capacities and dimensions across different cultures. Cross-cultural psychology is part of mainstream psychology to a greater extent than cultural psychology, and leading cultural psychologists like Shweder have accused it of merely exporting the Western version of general psychology to the rest of the world. The point of criticism is that we take Western personality theories, intelligence tests and fundamental psychological concepts and see how other cultures (typically East Asian) score on "our" tests. Cultural psychology, in contrast to cross-cultural psychology, would rather understand how people live, act, think, and feel from the perspectives of local social practices. Psychologists who have stood astride two different cultures at the same time report that it is difficult to find meaningful, simple comparisons or translations between psychological concepts in different cultures, for example, from Indonesian to American (Danziger, 1997). Folk psychologies can perhaps be outright incommensurable, i.e. largely incomparable if they differ in their basic parameters - if, for example, they disagree on the basic concepts of thinking, feeling and action.

This does not mean that we cannot undertake comparative studies of different cultures. But it does mean that we should bear in mind that these studies are always done from a specific cultural perspective. Shweder sought to expose three 
general codes that structure cultures' functioning and which help to determine how different cultures regard and give meaning to the mind, self, morality, community and suffering. The three codes that different cultures use to organize their worlds of meaning are: (1) based on an idea of autonomy, (2) based on an idea of community and (3) based on an idea of a natural, sacred order (Shweder, Much, Mahapatra, \& Park, 1997). These do not exist in pure forms, but (1) is clearly in the foreground in the United States, whereas (2) and (3) are in the foreground in India. In the case of (1), morality is conceived of as a system of rights that involves protecting the individual's freedom. The self is seen as a structure of preferences that the individual should be allowed to realize to the greatest extent possible. The social sphere is implicitly conceptualized as a market where services are freely exchanged between equal individuals. Code (2) is based more on so-called communitarian values such as duty and interdependence, and the self is seen as being embedded in a tradition-specific social community rather than being an autonomous individual. That is why people here have certain obligations that arise from the community (linked to family or region) regardless of what he or she might otherwise have in the way of preferences. The self is not a structure of preferences, but rather like an "official", says Shweder, because the individual is born into a particular "office" by virtue of his or her specific social relationships. Code (3) is associated with religious notions of the spiritual dimension of the self and often with an idea of the purpose of life consisting in reaching for the divine. The world is seen as a natural, sacred order that must be maintained and defended if threatened.

The much talked about conflict over the caricatures of the Prophet Mohammed in the Danish newspaper Jyllands-Posten in 2005 can be interpreted as an expression of a clash between two different worlds of meaning: the Western idea of autonomy, which is a significant background value for freedom of expression on the one hand, versus an idea of an inviolable, sacred order on the other. Many people in the West reacted with surprise at the violent reaction seen in several Muslim countries because it seemed incomprehensible that people could be so offended by a caricature drawing. The problem on the part of the West was perhaps that it overlooked the fact that the Muslims were not offended on their own behalf, but on behalf of God: for them it was God himself who had been violated, which is why it seemed important to restore the natural, sacred order. And the problem on the part of the Muslims was perhaps that they overlooked the fact that the newspaper did not intend to offend God - but rather to challenge certain human conceptions. The conflict escalated for many reasons, of course, not least for reasons of practical politics, but a major factor that made it possible for a few drawings to be able to set souls and flags on fire was without a doubt the very different worlds of meaning on metaphysical, psychological and moral levels.

Shweder sees key insights in all three codes, which promote different kinds of values. He is of the opinion that all the values involved are genuinely valuable, so it is not a case of some cultures being "wrong", but rather about weighing values differently. In addition to the fact that different codes, world views and 
cosmologies contain different folk psychologies and thus different perceptions of what the mind and morality consist of, they also include different explanations for human suffering. There are three major widespread metanarratives of suffering: (a) the biomedical narrative, according to which suffering is explained as a result of material events, (b) the moral narrative, which frames suffering as a consequence of a breach in the moral order, and (c) the interpersonal narrative that refers to magic, witchcraft or spirits as explanations for human suffering (Shweder, Much, Mahapatra, \& Park, 1997, p. 127). Most educated people in the West will probably agree to some variation of (a), but on a global scale only about $15 \%$ of the explanations for human suffering fall into this category, and the same figure also applies to the moral explanations (b). The largest category is (c) with $42 \% .^{2}$ In spite of modernization processes, secularism and education, the world is still very "superstitious" when seen through the eyes of Western science.

Incidentally code (1) and (a) go well together: man and his mind are material manifestations that can be treated medically, and we are also endowed with an intellect that gives us dignity and rights, but which nevertheless can be viewed as being a property of a material brain. Code (2) and (b) also go well together: individuals owe their existence to the community, and suffering arises when the individual violates the community. Code (3) and (c) also go hand in hand quite often: there is a fundamental cosmic structure that cannot be mocked or changed, and if one mocks it or tries to change it then he is punished by spiritual entities.

It is easy to lapse into extreme views when reading ethnographers' and cultural psychologists' descriptions of how different cultures relate to reality: that "the others" are rather superstitious, silly, dangerous or stupid, and that only "we" are right. This is the trap of ethnocentricity. Or that no one can be right, or at least more right, than others, when there are such different interpretations of the mental, cosmic and moral realms. Nothing is ever better than anything else, which is the trap of relativism. The cultural and historical psychologist Charles Tolman formulated a theory that may allow us to strike a sensible balance between these extreme views. His argument is based on a theory of practice and especially on the Hegelian view that our moral convictions can be derived from the nature of human practices that have arisen historically (Tolman, 2003, p. 44). According to this theory, everything that inhibits our expression of practices is a breach of the common good of the people; that is, the values that go beyond cultural differences. A practice is defined specifically by Tolman as the joint provision of basic human needs through the use of mediating tools (p. 43). Common goods or values arise in and with our collective practices that are dependent on trust, cooperation and justice in order to function. Common goods do not have value because we like them, but we should like them because they are fundamental to important and basic dimensions of our lives. According to Tolman, maintaining and developing social practices as such is therefore a universal value against which all our particular notions of morality and value can be derived and justified.

The philosopher Anthony Holiday (1988) developed a similar theory based on the philosophies of Wittgenstein and Marx. Regardless of the many cultural 
differences that exist in regard to morals, folk psychology, metaphysics etc., there are certain necessary conditions that must be in place for cultures and societies to be able to exist at all. I will not delve deep into the defence of a universal moral basis for human society, but Holiday analyzes three moral values in detail: veracity (telling the truth as a moral value), justice and respect for rituals. He argues persuasively that no society could exist as such, if there were no practices in these societies whose function was to protect these values. If mental phenomena in the full human sense are dependent on a network of practices and their norms, and if norms are ultimately founded on universal moral values, then the possibility of mental life as we know it in humans, is in reality only conceivable on the basis of a common morality (I argue much more thoroughly for this in Brinkmann, 2011a). Now we are back to Aristotle: the city is man's teacher (and of course women's too), and there are certain moral values that every city (that is, every society) should build on and cultivate among its citizens. Far from all psychologists will go along with this thesis. My main concern has not been to prove this thesis once and for all, but simply to show that recognition of cultural diversity with respect to morality, folk psychology and basic cosmology does not automatically lead to either ethnocentrism or moral relativism.

\section{The mind in the family}

Of course, it is not just the "city" that is "man's teacher" - not even the "city" in the modern sense of socially organized education through the school system - but also the family. For a society to survive over time, numerous functions need to be attended to: reproduction, so that deceased individuals can be replaced by new ones; production, so that physiological life can be maintained; social order, so that destructive conflicts are avoided; socialization, so that new community members can become competent actors in the community; emotional support, so members feel that they are part of a community, especially in crisis situations (Berk, 1997, p. 540). Early in human history, all of these functions were probably taken care of by the family, but as society evolved towards greater and greater complexity and there was a greater division of labour, the family could no longer manage taking care of all of these functions. In modern society, production has been transferred to the business sector and maintaining social order has become a state responsibility, and the state has gained a monopoly on violence, and a large part of socialization is turned over to specialists in pedagogy and teaching. Reproduction and emotional support on the other hand are largely still functions that are carried out in and by families. It is therefore not surprising that the family has been regarded as an absolutely central arena for the development of the mind. Freud attributed the immediate family an almost overriding role in terms of mental development in the individual's earliest years of life, whereas later researchers regard the family as but one important arena for mental development among several others.

Everyone has a mother and a father in a biological sense, and most people are brought up in a family. We could say that the family as an institution is situated at a 
level between the individual and society, but this is somewhat misleading because society is not a "thing" out there in the world. It is more accurate to say that families are an essential part of society; that families are a way in which societies are organized in probably all known cultures. For most individuals, family provides the introduction to the norms and practices that are a prerequisite for the development of a mature mind. Some novelists have imagined a family-free society, such as Huxley's Brave New World, which incidentally are almost always portrayed as a dystopia, but in the real world there are hardly any communities or cultures that are not organized into families.

The fact that all cultures have familial institutions does not mean that the nuclear family is universal. This is a social-scientific myth (Valsiner, 2007, p. 172). There is considerable variation throughout the world in terms of the types of families that can be grouped by marriage relationships: monogamy, where one woman is married to one man; polygyny where one man has more than one wife; polyandry, where one woman has more than one husband; and polygynandry, which is group marriage where more than one man is married to more than one woman. Polygamy and monogamy are the most widespread forms of relations, and researchers estimate that $80 \%$ of all societies have allowed polygamy (p. 193). The argument that is sometimes heard that the nuclear family - one father and one mother who live with their children without other family members - should be the most natural (and therefore the most correct!) way to live is therefore incorrect. Viewed historically, the nuclear family is an exception that has nevertheless been portrayed as normatively proper and natural in the West. Nor is it true that children necessarily prosper best when they have a close relationship with only one person in a nuclear family, e.g. their mother. There is no research to suggest that young children are naturally "programmed" to enter into a close relationship only with their mother, with the father taking on a role of protecting the nuclear family from the outside world (Dencik, Jørgensen, \& Sommer, 2008, p. 207). Children have close ties to the person who cares for them regardless of their gender.

Nevertheless, the nuclear family is widespread today in Western societies, and if you look at it statistically, it is not in peril, although it has often been declared dead. The modernization and individualization of society does not lead to the dissolution of the nuclear family, but, on the other hand, we are witnessing great changes within the nuclear family as an institution (Dencik, Jørgensen, \& Sommer, 2008, p. 188). Although fathers still work more hours outside the home on average than mothers, and although mothers still spend more hours at home on housework and child rearing than fathers, the modern family is characterized by changes in gender roles, which is also of significance for children. Men and women today have to renegotiate the heterosexual family contract (p. 160). It is not certain what mothers should do and what fathers should do - even if the end result is often not so different than in earlier and more traditionalist societies. This is primarily a liberating and positive development that allows men and women to adapt flexibly and with equal opportunities for both sexes, but we must also be aware of the risk of confusion and negotiation problems that may be associated 
with the disappearance of fixed norms. Well-functioning families are able to create stable and secure conditions for mental development, where all participants children and adults - are heard and respected, but where adults are in charge of the important decisions and are thus responsible for them.

The mental development of children in families is a complex and contextspecific process. Nevertheless, psychologists have attempted to draw general conclusions about how parenting styles affect the development of the mind. In a slightly simplified way, it is possible to distinguish four ways of rearing children using a combination of two properties: demanding and responsive (authoritative), demanding and non-responsive (authoritarian), non-demanding and responsive (permissive or laissez-faire) and finally non-demanding and non-responsive (uninvolved). A demanding style is one that, by its nature, places demands on children and seeks to influence children's behaviour. A responsive style is one that listens to and enters into discussions with children. If you boil the extensive research on parenting styles down to a short summary, then it turns out that the most positive benefits for children in both the short and long term are associated with the first-mentioned authoritative style, in which parents are both demanding and responsive, i.e. they make demands, but at the same time they listen and are accepting (Berk, 1997, p. 546). Children who grow up in families that are characterized by the authoritative style tend to be happier, have greater self-control and achieve greater academic success. In comparison, children in authoritarian families tend to be less happy and are more reserved; children in permissive families are more impulsive, dependent on adults and do less well in school, while children with so-called uninvolved parents often lack basic social skills and also do less well in school.

These general comparisons are based on large studies and averages and do not say anything about individual cases. This means that we cannot know whether an authoritative style in a particular case will yield a fruitful result for that particular child, and of course we also cannot use backward reasoning and conclude that a child who lacks impulse control and performs poorly in school must have grown up in a permissive family. One thing we can be quite sure of, however, is that the possibility of developing mentally, i.e. developing the abilities and dispositions that enable one to participate competently in social practices, is cultivated in the family. A major mental capacity is the ability to distance oneself from here-andnow situations, and the development of this ability is probably best supported in authoritative family relationships where children are seen and heard, but not forced to take on responsibilities that they cannot manage yet. In my opinion, there is a tendency these days to give children responsibility for a lot of things that they are not yet able to be responsible for. For example, there is talk about children being "responsible for their own learning" and parents are eager to expose their children to early school-related learning in the hope that they will go on to perform well in school. In addition to the problem of being given responsibility for something they are not ready for, it is interesting to note that a good deal of research suggests that successful school preparation can actually be destroyed by exposing toddlers to school-related learning prematurely (Dencik, Jørgensen, \& Sommer, 
2008, p. 242). There should be warnings against pushing scholastics into early childhood, when children's primary task is not to learn letters and numbers, but to play, test out different roles and thereby make sense of social relations, social order and social complexity. This is arguably the best way to develop the child's mind.

Mental development consists of individuals increasingly mastering social codes and signals themselves, which they can master only in social situations with the support of more competent individuals, typically adults. In this sense, mental development consists of internalizing social patterns and gradually becoming a responsible actor among other responsible actors. In Denmark, the age of criminal responsibility is 15 years, which means that we then say that people have developed sufficient mental maturity to be accountable for their actions at this point. And at the age of 18 people are given the right to vote and are thereby formally included in the basic democratic process. I do not know whether these age demarcations are optimal, but it is clear that mental development takes place at different rates for different individuals, and depends especially on the social and family environment they have been given. Therefore, having formal limits for responsibility from a specific age that apply to everyone is a somewhat questionable practice. But the question, of course, is whether it can be done any differently.

\section{Conclusions}

This chapter has brought us into many aspects of cultural psychology. Its basic message is that man is a social animal in a very elementary sense. As creatures with minds, humans cannot be conceived without culture, socialization and social interaction. Acting persons can exist only within such societal contexts, since we are what Aristotle called political animals. And sociality cannot be conceived without some basic values and normativities that are embedded in the practices in which we participate. A substantial portion of the practices that are very important for mental development are organized in family structures. But are there no other conditions for the social realm besides values and social practices? Yes, there are, and the next chapter is about things and artefacts as a necessary condition for the existence of complex social relations - and thus a condition for the occurrence of mental life in the human sense. Society and sociality are not free-floating social relations, but are largely held together and stabilized by the material objects that surround us. The same applies to the mind and its capacities in more general terms.

\section{Notes}

1 I put "Western" and "Eastern" in quotation marks because both categories represent a dramatic simplification and both cover very large differences within each category. Despite this fact, a great deal of cross-cultural psychology uses such a simple dichotomy. See for example Heine for an analysis of "Eastern" and "Western" self-concepts (Heine, 2001).

2 The sum is not $100 \%$, presumably because some of the explanation types could not be attributed to the established categories. 


\section{Chapter 5}

\section{The mind and things}

We have seen that the mind in the traditional (but also highly problematic) Western perspective is perceived as something "inside the head" - either as "an inner world" in the form of ideas, as in the philosophies of Descartes and the British empiricists, or as "mental representations" in much of the modern cognitive sciences. We have also discussed a number of arguments for expanding the boundaries of the mind from being something inside the head to also include the body and the social realm. In this chapter, I argue for a further extension of the mind's boundaries to also include material things, objects and artefacts. The mind extends not only to the social world of practices, relationships and symbols, but also to the material world of things and technologies. Or, in other words, it is unhelpful to depict the world as divided into a social or cultural realm on one side and a material realm on the other. There is just one world, and the parts of the world, where human activity is significant, is at one and the same time social and material. What does that mean?

Let us consider the following example as an introduction to the topic: the ability to count and calculate can be said to be a prototypical psychological process. As children we learn to count and eventually to do sums. Initially we learn perhaps by rote memorization, including counting from one to ten as a kind of recitation and saying "two plus two equals four". Later we learn more genuine mental arithmetic. "Mental arithmetic" is precisely something that can be done using the head alone. Not because the numbers are "inside the head", but because you can add numbers together without others knowing that you are doing it. But we can also learn to count on our fingers. This expands the range of possible mathematical operations for children. It is easier to add and subtract when you can see the amounts in front of you, represented by your fingers. In this moment, your fingers are part of the cognitive or mental process of working out mathematics problems. They play an equal part in working out the problems as do processes in the brain, which of course are also necessary. You can even take it a step further, like my own son, and use your nose to count your fingers with by pointing to each finger with the tip of your nose. He found this necessary when he was using all of his fingers on both hands to represent amounts so he could not use them to count with! Later we learn more complex forms of calculation using mathematical symbols, 
which we write down using pencil and paper. Can we say that the pencil and paper then become part of the mental process, aligned with the involved processes in the brain?

Yes - why not? At least this is the argument of philosophers such as Andy Clark and David Chalmers (1998), for example. It is certainly difficult to find good reasons to deny that these things are mental, other than insisting that this does not fit our dogma that the mind is "inside the head". What about when we learn to use a calculator? Does it matter whether the "processing power" is outside the head (in the calculator's chips and circuits) or inside the head (in the brain's neural circuits)? No, not according to Clark and Chalmers. They say that in principle it does not really matter at all. We cannot draw a line between the mind and the world based on an inner-outer understanding. The mind (as an array of skills and dispositions) is in the world, and the world is in the mind (because the relevant skills and dispositions demand a material world in order to be enacted). For example, when we talk about calculating mathematically, the fingers, nose (!), paper, pencil, calculator and many other things, from a functional and ontological perspective, play an equal part in the mental process of working out mathematical problems as brain processes do.

In this chapter, I will elaborate this perspective on the importance of things for the mind. I will talk about "things" in a very broad sense. Other words, such as artefacts or objects, could have been chosen, but the more everyday word "things" is perfect, in my opinion, and highlights the fundamental material dimension of the objects that are important for the mind. The things that are important for mental processes are primarily technologies. Technologies are things that are combined with a practical rationality in human practices (see the previous chapter on social practices). A calculator is thus a material thing, but it is also part of a calculation technology that enables people to perform all kinds of mathematical operations. A piece of paper is a thing, but when the paper is part of an intelligence test, it is part of a powerful social technology that can allow or prevent access to certain social positions and sort people into special education classes, the Military Academy or the director's chair.

I will thus use the term technology in a very broad sense. Technologies do not just include computers and drilling platforms but also, for example, things like shopping lists, speed bumps and psychotherapies, which are also examples of technologies. The latter three belong to what I will call mental technologies, social technologies and technologies of the self, respectively. This distinction is somewhat arbitrary, and it could be drawn differently, but I introduce it here in the hope of being able to give a certain overview of the many implications that things and technologies have for the mind. Mental technologies are the things that we use to expand our ability to think and act in the world, i.e. our cognitive abilities in a broad sense; social technologies are the technologies that allow certain types of societies and social relations to be created and maintained; technologies of the self are technologies used for human improvement in a broad sense. Often, a given thing as a material object will be part of all three kinds of technologies. There is 
no sharp distinction among them. But as an ideal type, a shopping list is a mental technology because it enables us to remember which goods we need to buy. The shopping list itself is a material object that we take with us to the supermarket, and when we are confronted with its stimuli (in the form of characters we have scribbled down) we will be able to buy precisely what we need. Speed bumps regulate vehicle speed and thus help to regulate the complex social form of organization that we call traffic. And psychotherapy is a diverse technology of the self that trains humans in new and hopefully more productive habits, which ideally allows them to live better lives. These three types of technologies will be discussed in greater detail below as they are all important to the human mind. Finally, I will give an example of a type of object that I will call a symbolic resource, which is very important for our mental processes and which has aspects of a mental-, social- and self-technological nature.

\section{Mental technology}

In previous chapters we saw examples of approaches that very closely linked the mind to the concept of meaning. This applies to both the Wittgensteinian tradition and the pragmatic approach of Dewey, which understands the mind as the ability to respond to and engage in contexts of meaning. In this case the mind is normative, precisely because it is possible to respond more or less appropriately to a given context of meaning. A major discussion in philosophy in the twentieth century has been about whether meaning is in the head, e.g. in the form of mental representations, or not. If the mind is closely linked to meaning, then this is a relevant discussion for understanding the nature of the mind. Therefore, I will first briefly outline the discussion.

More than 40 years ago, the American philosopher Hilary Putnam delivered a highly controversial argument in favour of meaning not being in the head (Putnam, 1975). Putnam set out a thought experiment in which he imagined that our planet Earth has a twin, a twin Earth that is completely identical to ours except that the water on this twin Earth does not have the molecular structure $\mathrm{H}_{2} \mathrm{O}$, but instead has a molecular structure XYZ. All of the superficial properties of the water are the same in both places: It is usually wet and can quench the thirst, freezes at zero degrees Celcius and boils at 100, etc. Putnam now asks: if a person on Earth and a person on twin Earth, who is $100 \%$ identical, molecule for molecule, both think about water, do their thoughts have the same meaning? Are they thinking about the same thing? And Putnam answers in the negative. Because the person on Earth is thinking about $\mathrm{H}_{2} \mathrm{O}$, while his counterpart on twin Earth is thinking about XYZ. This is true even if they do not personally know the molecular structure of water. The point is that what they think about depends on the nature of the physical world and not on their knowledge or mental representations. So it is not a difference in people's "heads" that determines whether they think about the one thing or the other, but rather a difference in their physical surroundings. This thought experiment gave rise to the view called externalism: the meanings of our thoughts, 
utterances and attitudes are not determined by something inside the individual, but are instead based on properties that are outside the individual. Or, more generally: that the "contents" of the mind can only be understood by looking at the things that are "around" the mind (see also Noë, 2009).

The discussion about meaning, the Earth and twin Earth is ongoing, but other forms of externalism have emerged since Putnam started the debate. Putnam's externalism can be called semantic externalism because it principally concerns meaning and only indirectly the mind. One of the best known proponents of the externalist view today is the philosopher Andy Clark, and his externalism is not just semantic, but also psychological externalism. Clark argues that we humans are "natural born cyborgs". We use things and assistive technologies to expand and refine our cognitive processes (Clark, 2008). Everything from glasses that help us to see more clearly, to scientific instruments, books, charts, etc. are involved in human cognitive processes. Clark's psychological theory can more accurately be called "active externalism" (Clark \& Chalmers, 1998). Whereas Putnam's aforementioned externalism was passive, since neither the individuals nor the environment on Earth and twin Earth did anything themselves that made a difference between the two people in question, because it was just a static difference in the environment that made a difference in the meaning of their thoughts, active externalism focuses instead on how people and environments are actively and reciprocally involved in cognitive and, more broadly, mental processes. The conclusion is that cognitive processes are not in the head - at least, not exclusively.

This conclusion can be supported with a multitude of examples. Let us borrow from Clark and Chalmers and take a typical cognitive task that is often studied in experimental psychology as our first example: mental rotation. Mental rotation refers to the phenomenon that we can mentally imagine how three-dimensional figures will look from other sides that are currently hidden by rotating the figures with our "mind's eye". Let us now say you are given the task of determining whether a three-dimensional figure on a computer screen is the same as another figure that you can only see from another side, and you can either rotate the figure "mentally" or on the computer screen. Should we say that only the first situation involves something mental? Or what if future advancements allow you to have a mechanism implanted in your brain, a neural implant that allows mental rotation just like on the computer screen - except that it is "internal"? There are no good arguments for there being any fundamental difference between the three scenarios. Whether we rotate the figure "purely mentally", by using the computer screen or by using a neural implant is subordinate in the cognitive and mental sense. The mental technology that the computer program represents, and which makes it easier (for most) to imagine three-dimensional figures seen from the back than using the head alone, is as much a part of the mind in this situation as are the brain structures that normally enable mental rotation.

It is the same idea with a notebook, to use a more everyday example. If a patient with incipient Alzheimer's manages to cope with everyday life by noting relevant information in a notebook, then the notebook is, of course, a substitute for 
the "brainpower" that previously helped the person to remember, but there is no reason to believe that the notebook is any less mentally relevant simply because it is an external thing in relation to the skull. If someone asks the patient whether he knows how high Mount Everest is, and the patient says yes but can answer only by consulting the notebook, which correctly reads " 8,848 metres", then we have no reason to deny that the person indeed knows how high Mount Everest is. The notebook plays the same mental role for the person as memory plays for people whose brains work in such a way that they can use it (their brain) to recall the height of Mount Everest. A person who is not suffering from Alzheimer's may initially find it difficult to remember and say "Wait a moment ... I need to think about it . . okay, now I have it: 8,848 metres!" It is true to say that the person knows the height of Mount Everest the entire time, and it is also true to say that the patient who needs to look it up in the notebook to remember it knows the same fact the entire time.

A counter-argument could be that the notebook should not be regarded as part of the mind because it is physically separate from the person and can be located far away. You may lose the notebook, but you cannot lose your brain! In a sense this is obviously wrong, precisely because the Alzheimer's patient can be said to have "lost" part of his brain. It is also more generally questionable to require that something be physically carried on your person as a condition for it to be called mental. A person who needs to wear glasses to be able to make out the letters in a book can still be said to be able to read, after all, even if he forgot his glasses at the top of Mount Everest. In much the same way, a blind person's cane is a technology that enables an extension of his or her awareness of the external world, whether or not the cane is permanently attached to the person's arm or can be set aside.

Making physical proximity to a person's body the criterion for whether we designate a material thing as part of a person's mind does not really work. What matters instead is whether the thing plays a relevant functional role in relation to the person's ability to carry out specific mental tasks. However, this conclusion is not unproblematic, since the limit of things that can be said to belong to the mental realm must be expanded almost indefinitely because all kinds of things play a functional role - large or small - in almost every conceivable mental process. For example, the notebook we mentioned earlier is manufactured from a specific material that is processed by specific people through specific social practices. Should all of this be included in the person's mind? It sounds absurd on the surface, and herein lies the greatest challenge for the externalist perspective, which concerns delimiting the parts of an organism's environment that are relevant to the organism's mental processes. We can also play with a futuristic scenario where we are all constantly connected to the Internet, perhaps using a neural interface, where in a matter of milliseconds we can download all sorts of factual knowledge from a body of collective knowledge, a bit like when we use Google to find information today. Will everyone then know everything - that is, everything that is stored in the body of collective knowledge? It is hard to say whether we would talk about it in those terms if the scenario ever became a reality. But it is presumably true that 
there would be a number of revolutions in our perceptions about mental processes. I already use Google for a wide variety of tasks - to find factual information, as a dictionary, for help solving various problems - and when the network can be accessed from anywhere it will represent a huge advance in our cognitive abilities. Today, the Internet is a very significant mental technology for a large part of the population in the wealthiest parts of the world. Although it might sound crazy, in this sense the Internet can be considered as a part of our mind. Of course this also means that it will become increasingly difficult to separate the wheat from the chaff when our individual cognitive processes merge with the collective. It will be culturally and societally important from now on to develop methods to determine the credibility and usefulness of the information that we have access to at any given moment.

A further problem with active externalism follows from the previous chapters of this book. For I have argued that it is philosophically problematic to seek to localize the mind in the brain. But this is not just about the fact that it is the brain that is problematic as a location. No, localizing the mind in the first place is problematic. At least if we accept Aristotle's assumption that the mind is a range of abilities and dispositions, and not a thing. So "externalism" (the idea that the mind is extended and distributed over a collective world of things) is potentially just as problematic as, for example, neural "internalism" (the idea that the mind is in the head). The mind is not in the head, as the preceding chapters have hopefully shown, but in the strict sense it is not outside the head either, because it is not any "place" at all. The mind is a range of skills and dispositions to recognize the features of the world, solve problems, act and respond emotionally to what is happening, and as such it has some conditions or mediators, which I have tried to unravel in this book. The conditions, at least some of them, are located in physical space: the brain is located in the head, the body is located on a chair, etc. but the mind itself is not as easily located in a purely physically sense. Having said this, active externalism is still significant in that it reminds us that we should examine the specific things and technologies that surround us and which enable us to recognize a number of features of our world. This will always be a relevant exercise when we want to understand the mental life of humans.

\section{Social technology}

Mental technologies are a kind of cognitive technology that also enable various forms of human action, but which are mainly relevant for cognitive psychology. The thesis about "the extended mind" and active externalism were indeed developed within cognitive psychology, but there is a need to thematize the significance of things and technologies in other fields of psychology as well. This is particularly true for social psychology, which is about how humans live together in groups and communities. In the previous chapter on cultural and social psychology, I outlined some basic concepts within social psychology. Given that social psychology is about human groups and social relations, there has often been a distinct lack of 
understanding with regard to the significance of the world of material things. As the social scientist Bruno Latour describes it, it is often assumed in the social sciences, including social psychology, that our social aspect exists as a kind of world in its own right, or as a kind of material that has explanatory power (Latour, 2005). You often hear wording like "the person's actions were influenced by social circumstances" without it being clear what exactly about the circumstances makes them social. Latour accuses sciences that use social explanations like this of being mystifying. Explaining human actions with reference to "social aspects" or "the social context" is not really an explanation that teaches us anything, but is instead just passing the buck. This corresponds to explaining actions based on some kind of "social ether" that floats around and has a causal effect. It parallels mentalistic explanations of human mental life, which are often more mystifying than clarifying, like in instances where a person in the field of psychology says that a personality trait was the cause of a person's actions - though it remains unclear how a trait could cause an action. Latour's point is that we cannot begin to understand what social scientists call "the social aspect" if we do not involve the material world of things and technologies. Social life is held together by things - and not by ethereal "contexts" or the like.

Social life can be defined as the kind of life that exists when an individual actor can achieve a goal only through interaction with other actors (Latour, 1996). By this definition, many non-human species are social, which is now widely accepted (back in Aristotle's time he described bees and swans as being social animals). Especially the higher primates have fairly complex forms of social life, but, as Latour describes it, there are clear limits to how complex the social lives of animals other than humans can be. Among apes, every social action and social structure is dependent on the actors themselves - and nothing but the actors. The social constructionist descriptions of sociality, which neglect the importance of things for the social sphere, can really only grasp the sociality of apes and not humans, precisely because the social life of apes is not mediated much by tools and technologies. Apes therefore need to have constant contact with other apes in chains of specific interactions in order to maintain their "social" life. Apes need to be able to see each other, hear each other and touch each other to maintain the social order. The "social structure" in a group of apes is nothing but chains of interactions that are characterized in particular by fights for dominance and parental care. Human society, in contrast, is organized and stabilized by various forms of social technology, which means that human sociality can be much more complex than that of apes. Complex sociality in humans in this context results not only from a more advanced brain, but to a great extent also from our ability to maintain the social aspect through time and space by means of things and technologies. And the relationship between the advanced brain and an advanced sociality is a two-way relationship: not only are our advanced brains a condition for the development of complex social relationships, but the brain itself is a plastic organ that is formed in large part by the social influences to which it is exposed throughout its development. The human brain continues its development in "the social uterus" long 
after the baby is born. Not only does the brain affect social life, but social life also affects the brain.

Modern society is dependent on a variety of forms of technology to keep track of individuals and populations, including buildings, institutions, infrastructures, indexes, databases, legislation, parliaments and much, much more. These technologies are not random material things that people avail themselves of in social life. No, without such things, social life as we know it could not exist. Practically every human interaction involves things (Latour, 1996). And these things are not just a neutral means that we use to perform certain actions. This would imply that we had specific, solid intentions in advance of seeking to achieve these intentions via certain means. In reality things help to shape and constitute the intentions we can have. My intention of completing this book is possible only provided that there are books, alphabets and a publisher, and these are not just "means" that I can use to realize my desire, but which also help give shape to my desire. Latour uses the term "mediators" for things and does not just refer to them as "means". As mediators they form the goals they mediate. We can now see that the previous chapters on the brain, the body and society's social practices are exactly about how these are mediators of human mental life (Brinkmann, 2011b).

Because social technologies form what they mediate - and thus create our social life - they inevitably take on a political dimension. The social sphere is political in this sense. As per Aristotle, as rational, social animals we will also always be political animals. As an example of a social technology with political implications we can mention such an everyday item as a baby bottle. A bottle is a container that can be used to nourish babies if, for example, the mother cannot breastfeed or she does not want to. But the bottle also allows the father to be included as the one who feeds the baby on an equal footing with the mother. New social relations and forms of organization can potentially arise from this technology: the father can take paternity leave, even in the very early part of the child's life, homosexual fathers can potentially adopt since a nursing woman is not necessary to keep the baby alive. In principle, the baby bottle is a revolutionary technology that can radically change the relationship between the sexes. The technology does somethingit helps to mediate social opinion and enables changing social relationships, and thus it is political. Where theorists inspired by Marxism might be interested in how the spread of technologies depends on the applicable production conditions in a society, an approach based on social technology will focus just as much on how technologies affect the societies they are in. Put simply, Marxism explains things and technologies based on society, while Latour and like-minded peers explain society based on things and technologies. ${ }^{1}$ Or more precisely: they insist that social, and thus mental, aspects are basically something "artefactual" and technological. They insist on rethinking dualisms between nature and society, between the material and the symbolic. It is highly relevant to insist on this point in light of this book's purpose, emphasizing the following correlation: first, the mind is social, second, the social is technologically mediated to a great degree - which leads us to the conclusion, third, that the mind, at least in part, is technologically 
mediated. We cannot fully understand psychological processes if we do not understand that the reality of social technology is an essential condition and mediator of mental life. This means that the mind is political as well.

\section{Technology of the self}

The figure who perhaps most strongly emphasized the technological and political aspects of the mind in the broad sense is the French philosopher and historian of ideas Michel Foucault (1926-1984). Foucault was particularly interested in the technologies involved in the formation, discipline and socialization of individuals. He called a number of these technologies "technologies of the self" and he was particularly interested in the technologies of the self that the sciences of the mind had produced and propagated. It is a historical fact that psychology has produced technologies that have regulated humans' relationships with themselves to a great degree. Psychology has played a role in shaping society and the psychological subjects who inhabit it. Psychology - through its practical effects in therapy, mental testing and psychological counselling, and also through its promise of personal development in the school system, organizations, clinics and institutions - has greatly helped to produce people who understand themselves based on the tools that psychology has provided. Psychology has thus been a co-creator of its own subject: the mind (Brinkmann, 2008a).

Historian of psychology Kurt Danziger, identified how a certain way of existing as a psychological creature - namely, as a "laboratory subject" or "test subject" was developed in early psychological experiments and mental tests that have since proved to be of significant practical use, which explains why they quickly spread to a variety of practices outside the laboratory, especially in the educational system, treatment centres and businesses (Danziger, 1990). Here psychology was transformed from a system of scientific ideas into a system of practices - part of the impersonal disciplinary power that, according to Foucault, is involved in the management of the mind through measurements, statistics and scientific procedures. Today it is practically impossible to get a management position without taking a personality test, for example. Countless other tests have become widespread in society to measure and, where possible, regulate our mental characteristics.

Nikolas Rose's contribution to critical psychological history, by extension of this, placed a particular focus on exposing the practical technologies through which psychology has organized people's everyday lives and their relationships with themselves (Rose, 1999). From this perspective, we should not think about psychological theories as abstractions - as mere phrases in articles and books but instead as "intellectual technologies", or ways in which thinking is used in the world to make the world understandable and manageable in particular ways. We must therefore analyze the development of technologies of the self if we want to understand the history of psychology (Rose, 1996): considering that traditional psychological history usually reports about how psychology has increasingly gained ground in terms of neutral knowledge of who we are as psychological 
beings, then we should, based on a "human-technological" or self-technological analysis, be critically concerned to the same extent with whether it is worth being "who we are" (i.e. as subjects shaped by psychological technologies), or whether we should rather seek to change the technologies of the self that we have available: "Maybe our problem is now to discover that the self is nothing else than the historical correlation of the technology built in our history. Maybe the problem is to change those technologies (Foucault, 1993, pp. 222-223).

Foucault distinguished three areas in which people are made subjects by things and technologies (Foucault, 1994): (1) through scientific objectification, such as in psychology and psychiatry, (2) through "dividing practices", i.e. through distinctions between things such as sick and healthy, mad and sane, criminal and moral, and (3) through technologies of the self: ways in which people make themselves subjects, for example, by taking a self-improvement course or keeping a journal. Psychology, in part because of its heterogeneous nature and lack of a unifying paradigm, has had a special penetrating power, and has been able to enter into all three areas. (1) It has provided methods of scientific objectification of human characteristics; not just in the form of theories of a "psychological" nature, but especially in the form of practical technologies for measuring and calculating mental characteristics and individual differences among people. (2) It has produced a number of "dividing practices" that have been institutionalized, and which now seem indispensable in modern society. (3) It has - and this is perhaps its greatest cultural influence - developed a number of tools in the form of technologies of the self, which people can use to shape and reshape themselves as subjects: to "work on themselves", realize themselves, develop personally, find their inner child, have increased self-esteem, release their inner potentials in professional and private life, become more assertive, process grief and suffering and so on (Brinkmann, 2017). In particular, the many competing psychotherapies have conspicuously become technologies of the self these days. The therapies are involved in a very direct manner in the cultivation of certain psychological characteristics like autonomy, quality of life and self-knowledge.

Psychology has not only provided neutral forms of understanding the mind, but it has also been involved in reinventing the mind and our way of existing as mental beings (Hacking, 1986). One example that we can mention here is how psychology invented the multiple or split personality (Hacking, 1995). New memory sciences from the late nineteenth century created a new object of knowledge memory - as something we could gain knowledge about through measurement methods and psychological techniques, which in turn allowed the emergence of phenomena like trauma, post-traumatic stress syndrome, hysteria and Multiple Personality Disorder. Diverse cultural movements then arose, including outright interest groups that were built around opposition to child abuse, which was believed to be the underlying cause of Multiple Personality Disorder, which in turn affected people's understanding of themselves and their past. There is a "looping effect" between the scientific description of the mind and human actions and self-understanding (Hacking, 1995). There is an interaction between people's 
self-perception and the classifications and descriptions that they can take on, or have put on them. This interaction is technologically mediated. According to the philosopher and historian Ian Hacking, a person can be gay, autistic, a teenager, a high priest or a Dane only if these classifications and descriptive forms are discursively available at the particular time in history in which you happen to live. These classifications are dependent on a number of practices and institutions, and when they disappear, the possibility of being that particular kind of person disappears too. For instance, you can no longer be a samurai warrior, since the background practice for this category no longer exists, but you can be a stockbroker instead. The development of new descriptive forms and categories thus makes new actions and new intentions possible.

If this is true, then every study of the mind should be interested in the reflexivity that exists in the fact that new knowledge about the mind can change the mind itself. Briefly put, the mind is shaped by our attempts to gain knowledge about it. Unlike molecules, which do not read chemistry books, people actually do read psychology books and can alter their actions to a certain extent based on what the books say about them. You could learn to reinterpret your life in the light of a new diagnosis (e.g. ADHD) and hence change your living conditions. Or you can object to certain mental classifications that others put on your own life, which is what happened, for example, when gay people succeeded in removing homosexuality from the psychiatric diagnostic system. This became a reality as late as 1973 . It is people as mental beings that produce psychological science, but it is also to some extent psychological science that contributes to producing the mind through its technologies in pedagogy, education, therapy, testing and personal improvement in modern society. This technologically mediated interaction between the mind and the sciences of the mind is what the term "looping effects" refers to.

\section{Mental development through material and symbolic resources}

Not only human scientists like Foucault and Latour have been interested in the significance of things for the mind, but cultural psychologists have also analyzed mental development in light of things and artefacts. Analysing the pure "social interaction" between parents and children is not enough to understand how children develop. We also have to involve the things that help to organize children's everyday life, such as computer games, books, dolls, footballs, the way their rooms are laid out and their high chairs. In this last respect, it may at first glance seem entirely uninteresting, psychologically speaking, that in Western culture we typically put young children in high chairs at the dining table and feed them with a spoon. We often allow the child to use the spoon himself, and many of us gladly tolerate porridge on the table when the child is learning to master the movements his arms make. If we take a closer look at it, this is an example of a rich social situation that encourages certain forms of mental development to the detriment of others (Valsiner, 1987). Placing children at eye level with adults teaches them that 
they are active and equal participants in family activities, whereas feeding children while they are on a parent's lap to a greater extent signals passivity and the fact that the child is not on an equal footing in the dining situation. This material arrangement and the things it involves teach us about society and norms from the time we are young, entirely without our being aware of it. We are instilled with specific habits in dealing with people and things that are extremely difficult to change since they are embedded in our daily routines - see, for example, "Throwing Like a Girl" in Chapter 3.

One type of thing that is important for most people's lives and development is cultural products such as music, books, films and pictures. We often consume these cultural products without thinking about what we are doing. Often it is just to pass the time. But when we use such things with specific purposes in mind, they are called symbolic resources in cultural psychology (Zittoun, 2006). When studying human life, it turns out that symbolic resources are part of a great deal of developmental processes throughout life. The earliest use of symbolic resources might be songs that parents sing to their children, and later, for example, bedtime stories. Both types of symbolic resources function to reassure the child, i.e. to cause a change in their mood and create a safe and secure atmosphere. This is a use of cultural products that most people will continue to avail themselves of later in life, and most people are familiar with the experience of humming a tune to themselves when, for example, they are walking alone on the street at night and they are perhaps afraid. In this case people have internalized a particular use of the cultural product and they can use it to affect their own emotions. We can present ourselves with stimuli and often thereby bring about a desired mental change.

Mental development can be described as a result of life transitions, after which our experience is reconstructed with the help of language and other signs. For example, a transition occurs in children's development when they learn to walk; suddenly they can move about and they have to take new challenges into account, and a transition occurs in young people's lives when they leave home. Following a transition, people's experiences and mental functions need to be partially reorganized and symbolic resources are an essential tool in this respect (Zittoun, 2006). Traditionally, most cultures have had larger shared systems of meaning that involved organized uses of symbolic resources. In a predominantly Christian culture, it is clear that the Bible plays a major role as a symbolic resource that people turn to in order to make important life choices, to seek comfort, to understand the secrets of existence and to perform many other psychological processes. With widespread individualization and the breakdown of traditions, it has increasingly been up to individuals to seek out and use symbolic resources.

In the past, there were also more definite rites of passage in connection with these life transitions, where predetermined symbolic resources were involved in many ways, such as in baptisms, confirmations, weddings and funerals. Today, people increasingly need to act as bricoleurs and piece things together themselves, i.e. by assembling a repertoire of symbolic resources that can be used in connection with life transitions and with mental development. The process, 
which consists in living through a transition and then creating a future orientation through bricolage and a composition of symbolic resources, can be seen as the core of human psychology (Zittoun, 2006, p. x). Most people can describe the benefits they get from reading books or watching films when they try to find their way through life's questions, big and small. This is a very everyday way in which things, in the form of cultural products, form part of our mental processes in relation to thinking, acting and feeling.

Symbolic resources can thus function as a mental technology and allow specific ways of thinking, they can function as a social technology to organize social interaction, and they can function as a technology of the self - as a tool with which individuals develop themselves. There is no doubt that symbolic resources are a very important aspect of the formation of human identity, at least if identity is defined as the way a person understands himself and his life (Brinkmann, 2008b). Symbolic resources give us our bearings in life and help us to contemplate what is important and unimportant. For instance, when we need to make difficult moral choices in daily life, rarely do we consult a specific set of ethical rules. Instead we use narratives about certain people we find admirable as a model and we ask ourselves: How would the person I admire act in this situation? The person may be real, but might just as well be fictional, like a character from a novel or an action hero from a feature film. Psychologists and other scientists who study mental processes have begun to realise in recent years the degree to which these cultural products, which we are almost constantly surrounded by, play into our mental processes.

\section{Conclusions}

In this chapter, I have outlined three types of technologies and briefly examined their significance for the existence of mental life. The basis for the significance of things and technologies is radically different from the usual psychological ideas about the mind. The traditional perspective places the mind "inside the head". Identifying the mind with the brain is an example of this. The externalist perspective, in contrast, says that the mind can be understood as such only by involving the external world and its mediators. The mind is not confined within the skull, or inside the body's skin. The psychological sphere must be understood by involving material and social objects in the external world. We are - and always have been (as long as we have been people) - natural born cyborgs.

\section{Note}

1 Latour's perspective is known as Actor-Network Theory (or simply ANT) and is characterized by insisting that things, and not just people, can be actors who do something. ANT is one perspective within a larger group of theories called Science and Technology Studies (or STS), which studies the social conditions and implications of technologies and sciences. 


\section{The mind between the brain and culture}

We have now been on a long journey from brain research to phenomenology of the body to cultural psychology and an analysis of technology. The general argument has been that these areas are all essential for an understanding of the mind, and that not one of them is sufficient on its own. They are in different ways conditions for, and mediators of, the mind as a range of skills and dispositions to be able to develop. In this chapter, I will summarize the book's explanations of the neural basis for the mind and the cultural conditions for mental characteristics and processes, respectively. I will try to rise above the specific areas that were analyzed in the previous chapters, and attempt to integrate them into a kind of "hybrid psychology" that takes into consideration both the brain and culture, which are otherwise often regarded as contradictory and perhaps incompatible approaches to the study of the mind. Using Rom Harré's work as a point of departure, I will outline the contours of an overall hybrid psychology, and finally I will give some suggestions for the future study of the mind. In the next and final chapter I apply the theory to a specific phenomenon, viz. ADHD as an example of mental disorder that is currently heavily debated.

If only one conclusion is to be drawn from this book's chapters, then it should be that psychology needs more than one form of understanding and more than one level of analysis. The brain alone is not enough, nor is the body, culture or materiality. This is why the attempts at monopolization that we have seen in the history of psychology have been unproductive when they seek to dispel one of the two halves of psychology that were outlined in the beginning of the book the nomic or normative. Against this background we have to conclude that both psychologies - or rather both sides of psychology - are relevant and indispensable within its respective domain. But this is where the problem comes in: Can we have both of these psychologies at the same time? I think so, and in the following I will present a proposal for how this can be done, with inspiration from Rom Harré.

\section{The human grammars}

Harré's hybrid psychology seeks a peaceful coexistence between the two psychologies based on the natural sciences (nomic) and the cultural sciences (normative) 
respectively. In his book Cognitive Science, he starts off by outlining an increasing polarization in psychology (Harré, 2002). On the one hand, there are researchers who insist that psychological phenomena are discursive, meaning that the phenomena are constituted by meaning, intentionality and normativity and by the means we use to create and express meaning. These researchers are right, according to Harré. And on the other hand, there are researchers who claim that psychological phenomena are based in a materiality that is comprised of the brain and central nervous system. These researchers are also right, says Harré, and his task is to show how both poles in this polarization can be right at the same time.

Harre's thinking is in direct continuation of Wittgenstein. Therefore, the use of language and the practical circumstances in which language is given meaning form the chief focus in an understanding of the mind. An understanding of what Wittgenstein called grammar is also key. As previously mentioned, grammar is an open set of rules for how different systems of symbols are used correctly. Grammar is thus something normative, which expresses how we should use language and its signs and symbols if we want to express ourselves in a meaningful way. As I argued in Chapter 2, it is a grammatical point that we, for example, cannot meaningfully attribute psychological states to the brain. We can attribute these states only to living organisms that move intentionally - and some of them apply only to advanced organisms, such as humans.

Harré distinguishes four different grammars, which structure the way we meaningfully talk about the mind in our culture (see Harré, 2002, p. 148). He calls the grammars S (for soul), P (for person), O (for organism) and M (for molecule), respectively.

\section{Soul grammar}

S-grammar expresses the idea that every human being has a special substance inside themselves - typically in the form of an immortal soul. This grammar was particularly prevalent and significant a few centuries ago, and it still plays a role for many people today, though in general we have to say that it is less important now than it was in the past. This grammar operates with two kinds of active beings, specifically God and souls, and it identifies key characteristics of actions based on the concepts of sin, temptation, forgiveness and confession. These concepts have not disappeared from our language, even though the underlying grammar has become obsolete in many people's lives with the widespread process of secularization. The grammar allows certain actions and utterances to be meaningful (e.g. "God's grace saved his soul") and excludes others from being meaningful (e.g. "God weighs 87 kilograms, which is twice as much as my soul"). Given the fact that many people still think, act and feel on the basis of S-grammar, it is important to include it when we want to understand why people do what they do, but - according to Harré - this grammar no longer has any direct bearing on scientific psychology. The example of S-grammar suggests that grammars are not set in stone at all times, but they evolve over time and some may even perish. 


\section{Person grammar}

While many people today manage without S-grammar - both existentially and in terms of understanding their own and others' actions - none of us can get by without P-grammar. P-grammar expresses what we assume when we view other people as irreducible sources of actions. This is what we do when we relate to others as persons. If a person, for example, has the right to vote, then the person is regarded as an individual who has voting rights and who can put a tick on a ballot paper during general elections. We cannot meaningfully say that it is the person's brain or body that has the right to vote or that ticks the box. No, within P-grammar it is irreducibly the person. The concept of the person is irreducible because it is primitive i.e. it cannot be derived from anything else. A person cannot be reduced to something more primitive, such as a soul or a body, because people cannot be divided into separate elements. In this sense, people are the atoms of social life (the term "atom" comes from the Greek word for indivisible). And that is why people have no inner complexity. They themselves are the source of action - it is not just a part of them that is acting when they act, but the person himself who is acting. This is the core of personism, which has been defended throughout this book with inspiration from Sprague (1999).

P-grammar is closely associated with our important concepts and practices that involve responsibility, because only a person can be responsible. Dogs and babies are not responsible for what they do, because they are not persons (at least not yet, in the case of babies). At the same time, however, a condition for the development of personal status is that you are treated as a person before you actually are one. As we have seen, this can be described based on Vygotsky's understanding of development, where an intention (pointing at something, for example) is attributed to a child by adults before the child is actually able to point (children can only grasp out of pure reflex), but this is also a condition for children eventually learning to point intentionally. To become a candidate for having your actions described in terms of P-grammar, you need to be treated as a person before you are one, paradoxically enough. P-grammar is fundamental for most of the psychological concepts that we can apply to adults (it is by viewing them as persons that we can say that they go to work, build a house, play table tennis, celebrate Christmas, etc.). Even in terms of things like memory, which at first glance seems relatively simple and mechanical, P-grammar is presupposed when making meaningful statements. Remembering something means remembering it correctly - and only people can remember, and not, for example, their brains. If you say "Now I remember what happened", then you make yourself responsible for the authenticity of the memory, and responsibility is only meaningful in light of P-grammar (Harré, 2002, p. 148).

\section{Organism grammar}

O-grammar expresses the norms for how we can meaningfully talk about organisms, i.e. biological, living beings. Active beings in this case are not souls or 
people, but organisms. We can meaningfully say many things about organisms that we cannot say about things. If a stone rolls down a mountainside and starts an avalanche, then this process is normally described in purely mechanical terms. There are other factors at play if we talk of a mountain goat that runs down the same mountainside looking for a fresh tuft of grass. The stone falls because there is a specific causal process in motion that can be described based on the law of gravity. The mountain goat runs in order to find some grass. It therefore has a specific goal as an organism, in that it has certain needs for food, and this justifies our description of it as something more than just a thing in motion. O-grammar is an expression of the latter and more. Mountain goats, dogs and cats appear to be creatures that, on the one hand, seek to achieve certain goals through their behaviour (e.g. catching prey), but their form of intentionality, on the other hand, does not justify that we fully attribute responsibility to them and we will therefore not regard them on the basis of P-grammar. But the boundaries are fluid here and we may potentially think that P-grammar should be extended to the higher primates. The fact that there is no sharply defined boundary between P- and O-grammars does not mean that it is pointless to make a distinction between them. In fact, the point is that we cannot do without a distinction based on the way our conception of ourselves and each other works.

It is a fact that O-grammar has spread to more and more domains of human existence in recent years, especially thanks to successes in biological sciences. Behavioural genetics and evolutionary psychology are examples of scientific attempts to colonize areas with O-grammar, which have traditionally been understood on the basis of P-grammar. In this perspective, parents' love for their children is merely an expression of genes providing for themselves in a specific type of organism called offspring, as evolutionary psychologist David Barash has argued (Barash, 1982, p. 70). Maybe the same thing that happened with S-grammar in the past i.e. that it lost ground - is going to happen with parts of our P-grammar today. The majority of people today work from the assumption that people - and not a divine force - are responsible for their actions. But there are good reasons to believe that there are limits to the amount of P-grammar we can dispense with in favour of O-grammar (more on this below).

\section{Molecule grammar}

M-grammar is based on molecules and clusters of molecules as the basic active units. The molecular world can be described here as being of key importance in the area of psychology in broad terms. If a person's brain is dysfunctional in terms of producing neurotransmitters, there are consequences for the person's psychological capabilities in that they will not be able to think or remember as well. Most people make use of M-grammar in everyday life in various ways, such as when we explain the effects of alcohol on the central nervous system and thus its effects on human behaviour. We have seen that eliminative materialism believes that we should limit ourselves to using only M-grammar when we conduct psychological 
science, since this is the only strategy that will be in line with the current scientific world view. We have also looked at some problems that arise when we eliminate the other grammars, and arguments against this kind of reductionism or eliminativism are discussed further below.

In summary, we can say that $\mathrm{O}$ - and $\mathrm{M}$-grammars express natural (nomic) necessities while S- and P-grammars express semantic (normative) necessities. When we say that the former grammars express natural necessities, we mean that explanations based on these grammars look at human behaviour with the goal of being able to establish empirical laws which, we assume, must apply to certain material mechanisms (organisms or molecules). The fact (if it is indeed a fact) that lithium can have a moderating effect on mood swings associated with bipolar disorder (formerly called manic-depressive disorder) is a finding that makes use of these grammars. In this case we are dealing with a causal and empirical explanation that builds on the assumption of specific mechanisms in nature.

P-grammar, on the other hand, does not express natural, but instead semantic, necessities. That is to say, it does not express necessities that have to do with empirical facts, but rather those that have to do with meaning. If someone sincerely says that they are happy about receiving a gift, then this is an expression of happiness that the person cannot be wrong about. Saying that you like a gift is not a report on the happiness that you read from your inner mind (and which may be true or false), but instead is simply a personal expression of happiness. This happiness can be compatible - or not - with local conventions for expressions of happiness (and is therefore normative), but it cannot be true or false. This is not because of a natural necessity regarding happiness, but instead concerns the meaning of the term. In much the same way, it is not a natural necessity or an empirical law that people experience surprise in unexpected situations, as we previously saw. Rather, it is a semantic necessity arising from the fact that surprise is defined as that which occurs in unexpected situations (because if no one was surprised then the situation was not unexpected). The fact that people are surprised in unexpected situations is a normative or semantic insight - and not an empirical one.

In short, P-grammar applies to the discursive domain of human psychology, where thoughts, feelings and actions are normative, which is to say that they can be judged as being more or less adequate and correct in relation to local standards. O- and M-grammars apply to the conditions for the mind that can be regarded in a framework that explains causes. This does not have to do with the normative, but instead deals with what I called the nomic earlier in this book. Psychologists and others who study mental processes avail themselves of all three grammars: M-grammar is used for example within neuropsychology and psycho-endocrinology (the study of the significance of hormones for behaviour and personality). A simple physical causality may be at play here, for example, an increased amount of adrenaline in the blood having immediate effects on the body's muscles. O-grammar forms the basis for sociobiology and evolutionary psychology, where the main concern is studying the behaviour of organisms in light of evolutionary-based background conditions. Teleology is often involved 
Table 6.I The three grammars in psychology

\begin{tabular}{llll}
\hline P-grammar & $\begin{array}{l}\text { People are seen as } \\
\text { actors }\end{array}$ & Normative explanations & Cultural psychology \\
O-grammar & $\begin{array}{l}\text { Organisms are seen } \\
\text { as actors }\end{array}$ & Functional explanations & Evolutionary psychology \\
M-grammar & $\begin{array}{l}\text { Molecules are seen } \\
\text { as actors }\end{array}$ & Causal explanations & Neuropsychology \\
\hline
\end{tabular}

here, i.e. where we look at the purpose (from the Greek telos) that specific mental functions have. Certain features of organisms arise as a consequence of evolutionary pressure, and behaviour that is grounded in these features can be explained functionally, and not only based on primitive physical causality. Explanations that refer to functions are based on a different logic than those that refer to mechanical causes. And finally, P-grammar is fundamental for our folk psychology, for everyday explanations of human actions, and, for example, for cultural psychology and discursive psychology. We do not regard actions as causally generated events here, but rather as meaningful demonstrations relative to local standards for correctness. Explanations on this level will be genuinely normative and intentional. Some differences between the grammars are summarized in Table 6.1.

\section{Two principles for a hybrid psychology}

The three grammars provide a basic overview of the different ways that we can talk about psychological phenomena and they form the basis for the wide variety of approaches to the exploration of the mind. But have we come any closer to a hybrid psychology simply because we have this overview? I think so, but we still do not have clarity about how the three grammars can be integrated into a comprehensive hybrid psychology. Harré also has a recommendation for this based on two basic principles. We will follow his lead and call the first principle the tasktool metaphor and the second principle the taxonomic priority principle (Harré, 2002; Harré \& Moghaddam, 2012). Together they express how the grammars can be combined in a hybrid psychology and what kind of hierarchy we need to presuppose between them when we conduct psychological research.

\section{Task-tool metaphor}

The task-tool metaphor is based on the fact that we, as mental beings, must be described as active agents. We are engaged in ongoing projects in our lives, which we seek to realize, and Harré refers to these projects as cognitive tasks. These can be anything from writing a book to baking cookies or searching for an inexpensive hotel room. We need to avail ourselves of various tools in order to complete the tasks that we are engaged in. Some tasks require obvious tools in the completely conventional sense of the word. I cannot bake cookies without having access to 
flour, butter and sugar, for example, but I also need to have tools like a rolling pin and an oven. Some chemical reactions occur when the ingredients are mixed and baked, and these can be described in terms of M-grammar. By the same token, the mechanism of the oven can be described based on certain physical principles. But the thing that justifies the activity itself being properly described as "baking cookies" is not something that can be understood in terms of M- or O-grammar. It is a genuine human activity only when we view it in terms of P-grammar. Only here does it make sense to talk about baking cookies as being more or less successful. The fact that I, as the baker, seek to achieve a specific outcome (perfect cookies that are not burnt or inedible), means that normativity is involved in the practice. And if the cookies don't turn out well, you turn to the person who made the cookies to complain! It is not the oven or the flour that is responsible (of course, an oven can be broken, but we do not hold the oven morally accountable in such as case).

The point is that our tasks are always identified based on P-grammar, whereas the functioning of the tools is described based on O- and M-grammars. The tools can be described in terms of their organic and molecular properties, whereas the way people use them in order to perform certain functions must necessarily be described on the basis of P-grammar. Furthermore, the central assumption in hybrid psychology is that the brain is a tool for people's projects. The way the brain functions should be described based on the fact that it is an organ (O-grammar) that has a specific neurochemical structure (M-grammar). We must avoid committing the mereological fallacy of attributing characteristics to the brain that properly belong to the domain of P-grammar. Such properties can be attributed only to people, who use their brains to complete specific tasks and carry out specific projects. A description of cookie baking as a hybrid psychological process will thus include a description of the functions of the various tools (ingredients, oven and brain) based especially on M-grammar, which, when we look at the process in terms of P-grammar, are all used by a person who seeks to achieve a specific intention (baking cookies) relative to specific local norms for well executed cookie baking. The hybrid psychological assumption is thus that there is no contradiction between describing the brain's functioning causally (e.g. based on M-grammar) and the person's actions normatively (based on P-grammar), but rather that these are two complementary descriptions of the same process.

\section{The taxonomic priority principle}

Having said this, we still need to add something else which is just as important because, even though a hybrid psychology manages to bridge the gap between the different ways of describing things at the same time, this does not mean that the ways of describing things are equal. When we are dealing with the mind, the descriptions of people's actions, thoughts and feelings based on P-grammar are primary - and the other descriptions are secondary. This is the key personist 
principle. The understanding of the tasks is primary and takes precedence - and the understanding of the tools is secondary, if we are to remain within the tasktool metaphor. It is precisely this relationship that the taxonomic priority principle identifies.

The taxonomic priority principle says that we necessarily point out material tools and mechanisms based on criteria that are derived from the meaningful, human world of cognitive tasks and projects. This means that the side of psychology that works based on $\mathrm{O}$ - and $\mathrm{M}$-grammars is always piggybacking on the side of psychology that works based on P-grammar. Or, in more positive terms: the causal and functional approaches in psychology are necessarily developed on the premises of normative psychology. P-grammar is primary, and O- and $\mathrm{M}$-grammars are secondary. The principle thus emphasizes the fact that a tool is inevitably defined relative to the tasks that it can be used to perform (Harré, 2002, p. 138). We cannot understand what a tool is used for based solely on the knowledge of the tool in isolation (only from the knowledge of its molecular or organic structure). It is possible to understand a tool only if we understand the social practices and life forms that give the tool its meaning. To emphasize this again: the functioning of the tool is understood causally, according to laws, or nomically based on O- and M-grammars. Tasks, by contrast, are understood normatively and in terms of intention based on P-grammar, where persons are to be understood irreducibly as acting based on specific normative reasons. The tools, the material mechanisms, are nomic, whereas the active agents operate normatively, i.e. they cannot be understood as elements in causal chains, but rather as people who can follow norms and rules in their lives and actions (and thus can also break, develop and rebel against norms and rules).

Let us return to the memory example. Memory as a psychological phenomenon is a direct part of the normative person grammar. Remembering is a cognitive task and is an important part of people's everyday lives. Remembering something means to remember something correctly - in a normative sense. And it is irreducibly people who remember, and not their brains, but people certainly could not remember anything if they did not have fairly well-functioning brains. If the brain as a tool starts functioning poorly, it is possible to resort to shopping lists, for example, as a memory aid, and, ontologically speaking, the shopping list will then be a part of the person's memory to the same extent that the brain is. It is merely a question of an external versus an internal memory tool that enables the given cognitive task to be performed. The taxonomic priority principle should thus serve to remind us that it makes sense to examine the molecular or organic basis of memory only provided that we have already identified certain people's actions as instances of what we call "remembering" (Harré, 2002, p. 160). We cannot start by putting people in brain scanners and examining the blood flow in specific cerebral regions unless we have already determined in advance that we want to examine what is happening in their brains when they try to remember something. 


\section{Conclusions}

In this chapter, I have referred to Harré's prospect for a hybrid psychology that integrates nomic and normative perspectives in the proper way. It is important, exciting and legitimate to investigate the nomic, say, the neural basis for memory processes and other psychological phenomena, but it makes sense only on the basis of a normative determination of what exactly memory (or another phenomenon) is - under the conditions of P-grammar. And correspondingly so with all other psychological phenomena: thinking, problem solving, emotions, reading and so on. The criteria for whether a person is engaged in one of these processes are not what a brain scan or a blood test shows, or any other organic or molecular reading for that matter. The criteria are taken from the everyday world of human life, where we avail ourselves of P-grammar to understand ourselves and each other. If we eliminated this world - and our way of describing it - we would not know what we should look for in a brain scan or a blood test. Only on the basis of P-grammar - where acting, accountable individuals are the starting point and where the mind is fundamentally comprehended as people's abilities and dispositions - can the remaining scientific grammars come into play in illuminating the mind. This is why personism should be foundational for psychology - in its hybrid version and otherwise. 


\section{Persons and disordered minds'}

In this final chapter, I shall move on from the general psychological discussions about personism and hybrid psychology and seek to apply the theoretical framework unfolded in previous chapters to what many people probably see as a most important field in psychology: mental disorders. Psychology may well be an interesting science, but in terms of use value, its legitimacy lies in being able to understand and treat mental disorders. As I have said, the personism defended in this book is best represented in contemporary psychology by what is known as cultural psychology (Valsiner, 2014). Cultural psychology is not monolithic, but rather happily internally divided into different perspectives that put emphasis on different aspects of human mental life: intentional worlds (Shweder, 1990a), artefact mediation (Cole, 1996), and semiotic mediation (Valsiner, 2007), for example. It is also quite influential in educational psychology and organization studies that focus on human life within historically developed social practices. It is curious to notice, however, how relatively little today's cultural psychology has to say about mental disorder, which is otherwise a key theme in psychology as a whole. Most theorizing on mental disorder in psychology has historically been dominated by psychodynamic, cognitivist and behaviourist schools, and more recently by the neurosciences. This is a mélange of mindist/mentalist and reductionist approaches to mental disorder, and the question is whether it is possible to develop a personist alternative.

It is well known that Vygotsky took a significant interest in abnormal psychology in children, or "defectology", as it was then called (Vygotsky, 1929), but browsing through today's authoritative sources on cultural psychology, such as The Cambridge Handbook of Sociocultural Psychology (Valsiner \& Rosa, 2007), leaves one with the impression that an interest in mental disorder and suffering, particularly in adults, is more or less non-existent. This is probably an unfair verdict, and there are certainly interesting studies of mental disorder here and there by cultural psychologists, but they are not in any way defining the field, nor does a well-developed theoretical account of mental disorder exist in cultural psychology today. This chapter will tentatively begin to articulate a theoretical account of mental disorder from a cultural psychological perspective by seeking to combine awareness of the brain and body with socio-cultural norms and practices without 
reducing mental disorder to either of these. In that sense, it may steer a course between essentialist models of psychopathology on the one hand, and radical social constructionist ones on the other. Attention deficit hyperactivity disorder (ADHD) in adults is referred to as an illustrative example, but the theory presented here has more general ambitions. Following the convention in much of the clinical literature, I begin by introducing a case of a person who has been diagnosed with a mental disorder (ADHD), before venturing into more theoretical discussions.

\section{Tom's story}

In order to have a concrete case to use as a springboard for the ensuing discussions, I shall introduce Tom. I first met Tom in the summer of 2013, when I began as a participant observer in a support group for adults diagnosed with ADHD (as part of the research project Diagnostic Culture, which studied the impact of psychiatric diagnoses - depression and ADHD are singled out as key cases - on individuals and society) (Brinkmann, 2016a). The group members meet for about three hours once every month. Tom is there almost every time, and I have also interviewed him in his home. I have conducted this fieldwork in order to understand how adults diagnosed with ADHD use the diagnosis in their own lives. They are not simply given the diagnosis as passive recipients, but are using it actively for a number of purposes (Brinkmann, 2014). Using a cultural psychological term, we can say that the category ADHD operates as a powerful semiotic mediator in their lives that enables them to interpret their past in a new light and develop specific hopes and possible developmental pathways for the future, while excluding others. Most of the people in the group (there are usually around 15 people present at a meeting) have sought out the diagnosis themselves, either because their children have been diagnosed (and they can mirror themselves in the children's problems), or because they have been through some sort of crisis in their lives (e.g. a stress related breakdown) that prompted a more thorough examination by the doctor and later the psychiatrist, which eventually led to the ADHD diagnosis. This is quite unlike the situation for children diagnosed with ADHD, who are very rarely (if ever) the ones who initiate a diagnostic process.

Tom, a man living alone in his mid-forties, has also wanted the diagnosis himself. When he tells his life story in an interview with me, he talks about a history of unruly behaviours, concentration problems, and also petty crimes in his late teenage years. He was adopted as a small child after having spent the first month of his life at an orphanage, and he talks repeatedly about his quite hefty temper. But there was no ADHD diagnosis in his school years, and he was simply seen as one of the "naughty boys". After the school years, he was able to hold a number of jobs, he still has good friends, and he also used to have a girlfriend with whom he lived. He describes himself as "an ADHD person who is functioning well". He seems quite determined in his choices and opinions. For example, when he discovered that there was a long waiting list before he could see the psychiatrist and obtain a diagnosis (paid by the state), he decided to pay for the psychiatric assessment out 
of his own pocket, and he was finally given the ADHD diagnosis about six months before I met him for the first time. He is still in a process of reading and learning about the diagnosis and trying to adjust his medicine to an optimal level. He grows his own marihuana, which he consumes occasionally (but does not sell to others), and psychiatrists would probably see this as "self-medication". He is currently unemployed, but, interestingly, his last job was as a support person for young people with various problems, many of whom also have the ADHD diagnosis, so he now knows the diagnosis both as a professional and as a patient.

Tom's story is in some ways typical (e.g. concerning his history and problems as a child), but in other ways it is (like all life stories) unique. When interviewing Tom in his apartment, eating cake and drinking coffee, I remember very clearly that I had the thought that this person is not mentally disordered. So why was he given the diagnosis? What does it tell us about our current "diagnostic cultures", in which more and more conditions are seen through the diagnostic lens that Tom received a psychiatric diagnosis? Two things about Tom's situation are particularly noteworthy in this context, and I shall use these as background information in the following discussion about the concept of mental disorder and how to approach this in personist and cultural psychological terms:

First, Tom is a very organized person. His apartment is perfectly clean and tidy, and he has neat to-do lists placed on the refrigerator. He has prepared carefully for the interview with me, e.g. by reading from a book on adult ADHD authored by a well-known Danish psychiatrist and a psychologist, and he has written down a summary of its central messages that he wants to show me in order to convey what ADHD feels like for him. The capability of organizing his life and daily projects goes against the diagnostic criteria for ADHD, where having "trouble organizing tasks and activities" and "is often forgetful in daily activities" are central. Tom is aware of this himself, but explains it by saying that "it is in order to avoid the chaos" that he has a high level of organization. In other words, he compensates so well - by using calendars and to-do-lists (mental technologies), and perhaps also his marihuana - that many of the symptoms of disorganization in fact disappear. The crucial question then becomes: If the symptoms disappear, is the diagnosis then still warranted? Diagnostic psychiatry has since 1980 (with the introduction of DSM-III) been based on an assessment of symptoms (rather than, say, an understanding of the etiology and underlying psychodynamics of the person), so, strictly speaking, it means that a disorder is only there if and when the symptoms are present (Horwitz, 2002). Paradoxically, this seems to imply that people's compensatory actions will remove the pathology. Second, Tom describes how his problems really emerged after an injury he suffered while he was in the armed forces. He was on a three-year contract and wanted to make a career in the military, but his leg was more or less crushed in a traffic accident, and he had to leave the military. Tom describes the forces as a very good place to work, and it seems evident that the symptoms of ADHD were not present in his life during this period. So, the next question becomes: Did Tom in fact have ADHD when he was in the military? Is it possible to have ADHD in an extremely organized context 
with clear hierarchies and task allocations? Do the social practices of the military provide an ecological niche, to use Ian Hacking's (1998) term, in which the cognitive problems otherwise associated with ADHD are eliminated?

The point of raising these questions is to say that how one answers them will depend on the theory of mental disorder that one (implicitly or explicitly) subscribes to. To simplify: Essentialists on the one side will say that if one has ADHD, then one has ADHD all the time and everywhere, because the disorder is constituted by certain core neurocognitive deficits. Most social constructionists on the other side will say that ADHD is a socially constructed category that medicalizes and pathologizes problematic behaviours, and they thereby seek to deconstruct all pathological "essences" associated with the diagnosis. I shall explicate these divergent standpoints further in the following, before articulating a hybrid psychological theory that is neither essentialist nor social constructionist (in a radical sense), but rather situational, relational, mediational and thus, I shall argue, more theoretically sound.

\section{Essentialist and constructionist approaches to mental disorder}

According to a number of leading analysts, the contemporary understandings of mental disorder in the West are heavily influenced by "biomedicalization" (Clarke \& Shim, 2010). Despite the fact that no simple biomarkers have been found for any mental disorder in psychiatry (Singh \& Rose, 2009), and even though diagnoses can be formulated only by evaluating and counting symptoms (and not through brain scans or blood tests), there is a huge interest among researchers in the neuroscience of mental disorder that trickles down to the public and its "folk psychiatry". As we have seen in this book, it is obvious that the brain is important in relation to mental disorders, but this does not mean that it is possible to diagnose a mental disorder by looking at a person's brain. The enormous interest in the neurosciences of mental disorder these years (Rose \& Abi-Rached, 2013) goes hand in hand with the success of Big Pharma, if not concerning the treatment of mental disorder (where the results are mixed to put it mildly, see e.g. Healy, 2012), but rather concerning the marketing and spread of pharmaceuticals against a number of conditions such as anxiety, depression, and ADHD. Often, the marketing employs an M-grammar concerning mental disorder by talking about deficient brain chemistry in analogy with insulin and diabetes.

Concerning ADHD specifically, the now standard account in psychiatry is one that approaches this condition as a neurocognitive deficit, resulting in inattention, impulsivity, and hyperactivity to varying degrees, which are seen as chronic symptoms that can be treated with stimulant medicine, but which can never be cured (Buitelaar, Kan, \& Asherson, 2011). The neuroscientific approach easily (however not necessarily) invites researchers to think in essentialist terms and look for the core dysfunctional brain mechanism that is thought to be involved in ADHD. Scores of brain imaging studies are now published every year, which are 
interpreted in light of quite different theoretical paradigms. Some theories follow Russell Barkley who states that ADHD is at its core a disorder of behavioural inhibition (Barkley, 1997), while others are in agreement with Thomas Brown, who argues that the deficit lies in the inner management system of attention (Brown, 2005). Maiese (2012) provides a very helpful comparative discussion of these essentialist models and their respective shortcomings. In either case, the ambition is to be able to pinpoint exactly what the alleged deficit is in essentialist terms. Perhaps ADHD is even a natural kind, some will say, i.e. a specific illness entity that can be defined in terms of necessary and jointly sufficient properties, which ideally leads to a "strict in or out classification of all individuals" (Kincaid \& Sullivan, 2014, p. 3). This, at least, seems to be the essentialists' dream, which, if realized, would likely lead to more valid diagnoses linked directly to the brain rather than behavioural symptoms.

Among a number of problems related to essentialism, I shall merely mention two: First, as I have already pointed out, no "essences" (in the sense of simple biomarkers) have in fact been found in psychiatry as a whole, let alone in relation to ADHD. Essentialism rests on what the historian of medicine Charles Rosenberg has called disease specificity, which refers to the idea that diseases are specific entities that have a kind of independent existence beyond their unique manifestations in sick individuals (Rosenberg, 2007, p. 13). The idea of disease specificity is so ingrained in medical practices today that it is difficult to imagine that it was once different. But, according to Rosenberg's studies, disease specificity is a cultural idea from the end of the nineteenth century that has enabled people since to imagine diseases as discrete conditions in an organism, which can be defined and separated relatively clearly from other diseases, and which are therefore identical with similar conditions in other organisms. To simplify: Before the nineteenth century, there were sick people, and after there are actual diseases. Although the idea of disease specificity might apply to somatic medicine, where examples such as cancer or diabetes can serve as obvious illustrations, it seems quite problematic in psychiatry.

Second, and possibly even more detrimental to essentialism, is the sociological insight that disorders "are only intelligible due to the normative and social context in which they are found" as Bowden has recently argued (Bowden, 2014, p. 422). In the terms of the present book, not just a (deficient) brain is needed in mental disorders, but also a context of social practices and normativity. Among philosophers of psychiatry today, there is widespread agreement that one cannot define anything as a disorder in the absence of social norms of normality and suffering (Horwitz \& Wakefield, 2007). Even if we grant that what is today referred to as ADHD is a ubiquitous trait in human populations across time and place (as some evolutionary psychologists will say), we still need social norms in order to establish whether this trait represents a disorder or simply is "a way of being human".

Such arguments have led some to the belief that all mental disorders are nothing more and nothing less than contingent social constructions. This, obviously, is the anti-thesis to essentialist accounts, which is often referred to as social 
constructionism. Social constructionist models of disorder dissolve essentialist definitions and claim that the whole range of problematic human behaviours represent disorders only because of social categorizations and not because of anything inherent in the people who suffer. Social constructionist models come in many versions that are more or less epistemologically and metaphysically radical, but they all "encourage the view that social values and priorities are the sole historical determinants of medicine, music, and marriage", as Church writes in her account of social constructionist approaches to mental disorder (Church, 2004, p. 393). Some, like Kenneth Gergen, seemingly prefer to eliminate the "deficit discourse" of mental disorder entirely, incarnated in the diagnostic vocabulary (Gergen, 1994), while others, such as the less radical Peter Conrad, argue that diagnosing people with the ADHD category is a species of medicalization that amounts to "social control" (Conrad, 2006). The point is that abnormal behaviours, such as the deviance labelled as ADHD, "is not inherent to the individual, the act, or the situation, but [is] rather a process in which certain alleged 'rulebreaking' actions come to be defined as deviance" (p. 1). In other words, for a social constructionist, there are no mental disorders "out there"; they are all in the eyes of the pathologizing beholder.

If the essentialists tend to forget that social norms are needed to establish anything as a mental disorder, it can be argued that the social constructionists conversely tend to forget that suffering individuals very often look for "pathologizations" to explain their problems. This was also the case in Tom's story recounted above. Tom has wanted the diagnosis himself, because he has lacked an explanation of his problems. With their eagerness to move beyond the individual to the social context, social constructionists not only fail to acknowledge that individuals may bring quite different problems into a context, but also that individuals may in fact be very positive toward the deficit discourses that social constructionists wish to eliminate for the sake of the diagnosed. It seems simplistic to say that ADHD, for example, is nothing but a matter of social control, just as it seems like a naïve dream to look for a never-changing "ADHD essence" in the brains of the diagnosed. To return to Tom, it is noteworthy that he has not had his brain scanned, and it is quite unlikely (although we do not know this for sure) that anything about his brain would appear abnormal. In any case, he functions relatively well in his everyday life. It also seems far-fetched to understand his problems in terms of social control. He does have a history of problems, which, however, have waxed and waned as he has moved through different life contexts. At the same time, we no doubt need to take both social norms and practices into account when seeking to understand him and other people diagnosed with ADHD, and also the fact that these persons have brains and bodies that appear in certain ways (e.g. the restless movements of hands and feet that are characteristic of many people with the diagnosis) and are affected by the drugs in ways that many people (including Tom) appreciate (almost all the people with the ADHD diagnosis that I have met in my fieldwork use drugs such as methylphenidate and generally praise their effects, although there are exceptions). In my view, there is a need for an integrative 
understanding of mental disorders such as ADHD that is able to incorporate all these dimensions, and which steers a course between essentialism and constructionism. This is where the personist hybrid psychology of the present book might be helpful.

\section{A situational approach to mental disorders}

The Danish medical sociologist Dorte Gannik devoted much of her career to developing a situational theory of illness, which is deeply congenial to the project of the present book (Gannik, 2005). Her work, which was based on studies of somatic illness, notably back problems, is little known outside Scandinavia, but her theory is noteworthy because of its simple elegance. While it is certainly relevant in relation to somatic medicine, it seems to be even more to the point concerning psychiatric problems. For reasons similar to those I articulated above, Gannik rejected essentialist theories of illness and disease (although there is a conventional distinction between illness and disease, this is rendered problematic by her theory). She sees illness as something relational, "identical with a person's interactive relationship with her surroundings", and also as performative: "The theory abstains from approaching illness as something 'in itself' beyond those actions or reactions with which a person responds to everyday, bodily experiences (p. 332). She refers to the well-established fact that symptoms are pervasive in our lives, and most of us experience unpleasant sensations in our bodies every day. But symptoms are not diseases or disorders. She argues that we should talk about illness, disease, or disorder as something people "do" (perform, enact) in relation to physical and social environments. They exist only in and through the ways in which they are performed. This has also been argued by ethnographer and philosopher Annemarie Mol, e.g. in her study of artherosclerosis (Mol, 2002). Mol says that we should not think of diseases as "constructed", since this metaphor solely emphasizes human symbolic activities of social construction, thereby leaving out the body and the material world (p. 32). Instead we should use the metaphor of performance or talk about enactment. Objects such as disorders are enacted in practices, but this should not lead us to the view that they are simply done by discrete actors (who could just choose to do otherwise). Instead, the idea of enactment should suggest "that activities take place - but leaves the actors vague." (p. 33). Enactments presuppose a whole range of mediators that make the doing possible. If we ask "Who does the doing?" (e.g. of ADHD), then the answer has to include not just the suffering person, for events "are made to happen by several people and lots of things. Words participate, too. Paperwork. Rooms, buildings. The insurance system" (p. 25). And much more. The complexity involved leads to a need for what Mol calls a "praxiographic appreciation of reality" (p. 53), which studies how things are brought into being in socio-material practices (I refer the reader to her book to get an idea of how to work methodologically with this perspective). Contra essentialism, this means that it becomes impossible to isolate the 
"essence" of a disorder in any one place (e.g. the brain), and, contra social constructionism, it means that many forces besides the purely human (e.g. symbolic or discursive) ones are involved.

Returning to Gannik we can say that her model likewise does not isolate illness/ disorder to anything in the person as such (essentialism), nor to anything in the social system in itself (constructionism). Rather, illness and disorder are always found in a relation between a person (or organism) and life situations (constituted by socio-material practices). This relational perspective means that people's problems are radically situated. They exist in their concretely situated manifestations only, and not in anything behind or beyond this. Thus, essentialism fails. But it also means that the problems are irreducibly real - as real as anything gets - and sometimes stubbornly real. Thus, social constructionism in its radical versions fails. We should maintain a moderate social constructionist outlook, because certain important factors related to mental disorder are socially constructed (e.g. some norms inherent in our social practices), but we should also acknowledge the importance of factors that cannot be said to be socially constructed. It is the relation between these factors that should be in focus. Gannik's theory implies that treating people's disorders relationally may involve changing the person (e.g. through cognitive techniques or drugs) or changing the socio-material practices (e.g. by inventing new cultural prosthetic devices or new discursive practices). The point is that someone's problems are not to be located in a single place, but dispersed over multiple mediators.

If we look at Tom's story through this lens, we can see that his problems are not just socially constructed, and it seems unhelpful to claim that the ADHD diagnosis in this case is merely a species of social control. There is real felt suffering in his life, related to disruptive behaviours and a problematic temper, but, at the same time, this phenomenon seems to become ADHD only relative to certain sociomaterial practices and their norms, where the problem can be enacted as ADHD. A hybrid cultural psychology of mental disorders should ask what mediators are involved in a given case that makes this form of enactment possible (I provide a sketchy answer below). A situational approach to Tom's story also seems to imply that in certain contexts, such as the military, where the "symptoms" are not enacted, we should beware of concluding that he "has ADHD". Perhaps we need to conclude that Tom did not have ADHD in the military. From the situational perspective, one does not simply "have" ADHD (or any other disorder) here, there and everywhere - but enacts it only when certain contextual conditions and mediators are present. And even in life contexts where the condition can be enacted (such as in schools), we see someone like Tom being able to remove or at least diminish his symptoms with the use of culturally available technologies such as to-do lists. We may hope that future research into ADHD and related diagnoses does not only aim to develop new drugs to target brain chemistry (the brain mediator), but also the much more immediately significant (yet with much less prestige and money involved) everyday artefacts that may be used as cognitive "assistive technologies" (Gillespie, Best, \& O’Neill, 2012). 


\section{The mediated mind and its disorders}

If we follow Gannik and Mol and look for a framework between essentialism and radical constructionism, the question becomes: What kinds of mediators are involved that makes the enactment of a given disorder possible? And the practiceoriented question becomes: How can these mediators be changed in order to help persons? We have seen in this book that (1) psychological phenomena (our ways of acting, feeling, perceiving, and thinking) are normative in the sense that they do not simply happen, but rather are done (performed, enacted) by persons relative to social norms inherent in social practices; (2) that the mind should be thought of not as an entity, but as a name for the skills and dispositions that enable persons to enact psychological phenomena; and (3) that a range of mediators are involved in the constitution of this enactment. Some of the mediators are literally tools, while others, such as the brain, can be thought of metaphorically as tools (Harré, 2012). I shall now briefly discuss how the brain, the body, social practices, and material artefacts as mediators may be involved in the constitution of the ADHD phenomenon. Needless to say, I can provide only a sketch of the argument in this context.

Concerning the brain, it must be said that although no biomarkers for ADHD (or any other mental disorder) have been found that makes diagnosis possible, it is inconceivable for most researchers (including me) that the brain is not involved in constituting the ADHD phenomenon, for the simple reason that the central nervous system is active in relation to any psychological process imaginable. The brain is a tool that persons employ when they think, feel and act - or fail to do so in culturally sanctioned ways. A reductionist program in the neurosciences will study how thinking, feeling, and acting go on in the brain, but after reading this book it is hopefully obvious that only persons (and not their brains) think, feel, and act (sometimes in ways that are labelled "ADHD”), although they definitely need their brains to do so. Large research programs now investigate the brains of people with the ADHD diagnosis, and we will have to wait and see if something significant emerges (we know that the brain is plastic, which may mean that no common pattern - or essence - can be found in the brain across people diagnosed). In any case (and I consider this a quite trivial point), the brain is without doubt a mediator that is involved in constituting the ADHD phenomenon.

This also goes for the body. Not many researchers are concerned with the embodied nature of mental disorders, but Maiese (2012) is one exception in the case of ADHD. Based on a phenomenology of the body, she argues that ADHD represents a problematic bodily orientation through which a person interacts with and gives meaning to the world. She further argues that embodied affective framing mechanisms, which serve to help people understand what is important in different situations, are defective in people who suffer from ADHD. Nielsen has argued that bodies of people with an ADHD diagnosis are typically "out of sync" with the rhythms of the cultural world and interpersonal relations (Nielsen, 2017). It is difficult to assess the degree to which this perspective applies to Tom, in particular because of the ways that he has organized himself in his home, which 
renders his everyday life predictable and "rhythmic". But from meetings and conversations with many other adults diagnosed with ADHD, it is recognizable that some of them have trouble focusing on what for most other people is salient in a social situation and doing so in a way that is organized temporally in accordance with prevailing social rhythms (this sometimes leads to curious interpersonal misunderstandings).

Thirdly, and almost trivially from a social scientific perspective, is the way that ADHD is mediated by certain social norms as they are inscribed into social practices. Researchers have found, for example, that a child's date of birth is a very powerful predictor of whether or not that child will get the ADHD diagnosis: Studies from the US show that a boy born in January has a 70\% higher risk of being diagnosed than a boy born in December, because of the cutoff for being assigned to a grade (Frances, 2013, p. 141). What happens is that a child's relatively immature behaviours are pathologized as ADHD within the social practices of today's schools. With this, of course, I do not mean to imply that children really have ADHD because of their date of birth, but this finding certainly makes it evident that ADHD is co-constituted by norms about concentration and unobtrusiveness in modern society and specifically schools. In his account of the historical creation of ADHD, the historian Matthew Smith has even gone so far as to link the hyperactivity epidemic in the US with the "Sputnik panic" that emerged after the Russians succeeded in launching Sputnik in orbit around the earth, leading panicked American educators to reform US schools in order to be able to beat the Soviet Union, which allegedly had much more discipline compared to the reform pedagogy of John Dewey that otherwise had characterized the American school system (Smith, 2012). It is fascinating if global politics in this way leads to very local consequences for individuals who are diagnosed. For Tom, as I have tried to demonstrate, it is quite clear that his symptoms appear as problematic only outside the strongly organized context of the military, when he has had to lead an independent adult life, deciding every day what is to be done, when, and how. Modern life in the West has been described as involving a "tyranny of choice", celebrating the autonomous, reflective decision-maker in numerous social practices, and this may create ecological niches in which people are prone to being diagnosed with ADHD if they do not live up to the norms of constant free choice.

Finally, the fourth kind of mediator is artefactual, involving all kinds of technologies. For some disorders, it is obvious that mediators of this kind are involved in the constitution of the problem: Dyslexia, for example, is only possible because of the existence of written language. Concerning ADHD, it is interesting that the diagnostic criteria include such things as "fidgets with or taps hands or squirms in seat" and "loses things necessary for tasks or activities (e.g. school materials, pencils, books, tools, wallets, keys, paperwork, eyeglasses and mobile telephones)". In a world without seats (in school institutions), pencils, books etc. (which are representatives of "learning technologies" as such), and without the relevant socio-material arrangements of education in which bodies are socialized to a certain type of behaviour, it is difficult to imagine that ADHD could exist. 
Technologies may assist in the creation of disorders, but may of course also help alleviate symptoms, and I have already described how material technologies are used by Tom in attempts to regulate his life activities in various fruitful ways.

The point of listing these is to emphasize that the disorder cannot be isolated to any one of these sets of mediators, but must be seen as distributed between and across them. There is no essence anywhere that makes up ADHD in and of itself, but different mediators may be involved in different cases to constitute a person's problem as one of ADHD. Together, the mediators enable persons to enact their problems as ADHD: A working brain and body are needed, and so are social practices with norms and discursive categories, and also various artefacts (and I have not gone into details concerning the technologies of symptom check lists, pills etc.). Talking about enacting ADHD - or living under the description of "ADHD" (Martin, 2007) - is not to say that people do that consciously. Behaviours that we designate as ADHD are not chosen, but they are also not completely mechanical. I agree with Martin (who talks about bipolar disorder) that being hyperactive "does not fit easily at either end of opposites like conscious/unconscious, habitual/novel; compelled/chosen; or innate/learned" (p. 83). We need a new language, a new scientific approach to "describe the terrain between these poles" (p. 83), since this is where most disorders are enacted.

I can now sum up my argument by returning to briefly to Tom. If my argument is valid, we should see Tom's ADHD as mediated by his brain, body, social practices and various technologies. Contra essentialism, it is not isolated to any one of these, and, contra constructionism, ADHD represents much more than a (pathologizing) discourse. Hopefully, a hybrid psychological broadening of our approaches to mental disorder can also lead to innovative and humane ways of helping people with their problems. Sometimes, the best help is likely to be found by looking at a few of the mediators, but, in principle, I will claim that we should be able to address all of them. Cultural psychologists are particularly well equipped to study the mediators of social practices and technologies, but they should also be sensitive to the embodied nature of mental problems. It could be objected that Tom is a special case, and that other disorders manifest themselves much more systematically in people's lives. This is probably correct, but I do not think that it invalidates the general argument that cultural psychologists should theorize mental disorders as enacted relational, situational problems, related to a person's mediated mind.

\section{The future of the mind}

In this book, I have sought to convey a comprehensive answer to what the mind is, and thus a comprehensive theory of the mind, and in this final chapter I have tried to extend the theory to mental disorder. The argument implies that the future should belong to a pluralistic and cross-disciplinary approach to the mind that, on the one hand, entails a corrective to the current brain fetishism (and its reductionism) and, on the other hand, recognizes that the mind is biological as well as 
cultural (against radical constructionist perspectives). Human beings' mental lives are molecular, biological and meaningful to persons. In order to understand the mind in its totality, we need to speak in all three ways, which is to say that we need to use all three grammars that were introduced in the previous chapter: $\mathrm{M}, \mathrm{O}$ and P. But we must also keep in mind the personist point of departure that any study of the cultivated mind can take, and this is to be found in the personal perspective, where people are understood as intentional beings who can act based on reasons that can be more or less good.

A hybrid psychological understanding of the mind is therefore inclusive, but it is also aware of the fact that the psychological point of departure is always in our world of normativity. One way to put it is to say that (nomic) causal psychology always develops subject to conditions that come from an understanding of the mind's fundamental normativity. This applies even though it has been ignored or denied by a number of influential reductionist approaches to the mind in recent years. I have tried to treat these approaches as problems in need of a solution in this book, while rejecting a number of just as problematic - but very different approaches. Namely the mentalistic or mindist approach, which regards the mind as a mental world in its own right, which can be discussed independently of neural, physical, social and technological conditions. Such a world does not exist. The mind is not a "world", nor is it a "thing", but rather a range of skills and dispositions that exists in the world and which is identified on the basis of a normative order of human activities and practices. A number of different conditions are required for such abilities and dispositions to be able to flourish in life, and the individual chapters of the book (brain, body, sociality and things) have hopefully guided the reader in this direction.

Put positively, future studies of the mind will use this as their point of departure. In reality, scientific progress here will consist in returning to Aristotle's basic understanding of the nature of the mind. We need to go "forward to Aristotle", as Harré has put it (Harré, 1997). When we come forward to Aristotle we will realize that it is necessary to view things through both scientific and cultural lenses. We will understand that there is a need for both quantitative methods, which give shape to experiments and process the collected data statistically, as well as qualitative methods that seek to describe and understand human experience, meaning and norms. And we will be obliged to recognize that there is also an asymmetry in the way that the cultural-scientific and qualitative approach is a primary condition for the remaining ways to investigate the mind to get underway in the first place. Even though the qualitative understanding of the mind is supported only rarely by research grants or disseminated in the profession's leading magazines, this understanding is indispensable as a condition for being able to have a psychology at all.

From a more pessimistic perspective, we can fear that the fledgling hybrid psychology will not stand a chance, but instead will be trampled by the dominating neuro- and bioscientific approaches to the mind. These approaches represent the biggest trend in today's world of scientific psychology (Spear, 2007). And in both the media and in our daily lives, the biological perspective of the human being is 
also becoming more widespread (Rose, 2009). If my analysis in this book is valid, then there is reason to fear the imperialism of scientific, nomic psychology to the detriment of the normative and folk psychological understanding we have of each other, but there will also be clear limits to how far this imperialism can go. Our folk psychological and normative understanding of thoughts, feelings and actions may well be colonized for a period of time, but it can never disappear entirely.

\section{Note}

1 This chapter reworks materials from an article that has previously appeared in Culture \& Psychology (Brinkmann, 2016b). 


\section{References}

Aristotle. (1957). On the Soul. London: William Heinemann Ltd.

Aristotle. (1976). Nichomachean Ethics. London: Penguin.

Aristotle. (1978). The Politics. London: Penguin.

Baars, B. (1997). In the Theatre of Consciousness. Oxford: Oxford University Press.

Barash, D. (1982). Sociobiology and Behavior. New York: Elsevier Press.

Barkley, R. (1997). ADHD and the Nature of Self-Control. New York: Guilford Press.

Bem, S. \& Looren de Jong, H. (2006). Theoretical Issues in Psychology: An Introduction. (2nd edition). London: Sage.

Bennett, M. R. \& Hacker, P. M. S. (2003). Philosophical Foundations of Neuroscience. Oxford: Blackwell.

Berk, L. (1997). Child Development. (4th edition). London: Allyn and Bacon.

Bloom, F. E. \& Lazerson, A. (1996). Brain, Mind, and Behavior. New York: W.H. Freeman \& Co.

Bourdieu, P. (1980). Distinction: A Social Critique of the Judgement of Taste. (This edition 2004). London: Routledge.

Bowden, G. (2014). The merit of sociological accounts of disorder: The Attention-Deficit Hyperactivity Disorder case. Health, 18, 422-438.

Brinkmann, S. (2006). Damasio on mind and emotions: A conceptual critique. Nordic Psychology, 58, 366-380.

Brinkmann, S. (2008a). Changing psychologies in the transition from industrial society to consumer society. History of the Human Sciences, 21, 85-110.

Brinkmann, S. (2008b). Identity as self-interpretation. Theory \& Psychology, 18, 404-422.

Brinkmann, S. (2011a). Psychology as a Moral Science: Perspectives on Normativity. New York: Springer.

Brinkmann, S. (2011b). Towards an expansive hybrid psychology: Integrating theories of the mediated mind. Integrative Psychological and Behavioral Science, 45, 1-20.

Brinkmann, S. (2012). The mind as skills and dispositions: On normativity and mediation. Integrative Psychological and Behavioral Science, 46, 78-89.

Brinkmann, S. (2013). John Dewey: Science for a Changing World. New Brunswick, NJ: Transaction Publishers.

Brinkmann, S. (2014). Psychiatric diagnoses as semiotic mediators: The case of ADHD. Nordic Psychology, 66, 121-134.

Brinkmann, S. (2016a). Diagnostic Cultures: A Cultural Approach to the Pathologization of Modern Life. Farnham: Ashgate. 
Brinkmann, S. (2016b). Toward a cultural psychology of mental disorder: The case of attention deficit hyperactivity disorder. Culture \& Psychology, 22, 80-93.

Brinkmann, S. (2017). Stand Firm: Resisting the Self-Improvement Craze. Cambridge: Polity.

Brinkmann, S. \& Kvale, S. (2015). InterViews: Learning the Craft of Qualitative Research Interviewing. (3rd edition). Thousand Oaks, CA: Sage.

Brinkmann, S. \& Tanggaard, L. (2010). Toward an epistemology of the hand. Studies in Philosophy and Education, 29, 243-257.

Brown, T. (2005). Attention Deficit Disorder: The Unfocused Mind in Children and Adults. New Haven, CT: Yale University Press.

Budd, M. (1989). Wittgenstein's Psychology. London: Routledge.

Buitelaar, J., Kan, C. C., \& Asherson, P. (2011). ADHD in Adults: Characterization, Diagnosis, and Treatment. Cambridge: Cambridge University Press.

Cahan, E. D. \& White, S. H. (1992). Proposals for a second psychology. American Psychologist, 47, 224-235.

Chaiklin, S. (2007). Kulturhistorisk psykologi. In B. Karpatschof \& B. Katzenelson (Eds.), Klassisk og moderne psykologisk teori. København: Hans Reitzels Forlag.

Chomsky, N. (1966). Cartesian Linguistics: A Chapter in the History of Rationalist Thought. New York: Harper \& Row.

Church, J. (2004). Social constructionist models: Making order out of disorder - On the social construction of madness. In J. Radden (Ed.), The Philosophy of Psychiatry (pp. 393-406). Oxford: Oxford University Press.

Churchland, P. M. (1981). Eliminative materialism and the propositional attitudes. Journal of Philosophy, 78, 67-90.

Clark, A. (2008). Supersizing the Mind: Embodiment, Action and Cognitive Extension. Cambridge: Cambridge University Press.

Clark, A. \& Chalmers, D. (1998). The extended mind. Analysis, 58, 7-19.

Clarke, A. \& Shim, J. (2010). Medicalization and biomedicalization revisited: Technoscience and transformations of health, illness and American medicine. In B. A. Pescosolido, J. K. Martin, J. D. McLeod, \& A. Rogers (Eds.), Handbook of the Sociology of Health, Illness, and Healing (pp. 173-199). New York: Springer.

Cole, M. (1996). Cultural Psychology: A Once and Future Discipline. Cambridge, MA: Harvard University Press.

Conrad, P. (2006). Identifying Hyperactive Children: The Medicalization of Deviant Behavior. (2nd expanded edition). Aldershot: Ashgate.

Costall, A. (2004). From Darwin to Watson (and cognitivism) and back again: The principle of animal-environment mutuality. Behavior and Philosophy, 32, 179-195.

Coulter, J. (1979). The Social Construction of Mind: Studies in Ethnomethodology and Linguistic Philosophy. London: Macmillan.

Coulter, J. (1989). Mind in Action. Oxford: Blackwell.

Coulter, J. \& Sharrock, W. (2007). Brain, Mind, and Human Behavior in Contemporary Cognitive Science: Critical Assessments of the Philosophy of Psychology. Lewiston, NY: The Edwin Mellen Press.

Crick, F. (1995). The Astonishing Hypothesis. London: Touchstone.

Crowell, S. (2009). Husserlian phenomenology. In H. Dreyfus \& M. Wrathall (Eds.), $A$ Companion to Phenomenology and Existentialism (pp. 9-30). Oxford: Wiley-Blackwell.

Damasio, A. (1994). Descartes'Error: Emotion, Reason, and the Human Brain. New York: Quill. 
Damasio, A. (1999). The Feeling of What Happens: Body, Emotion and the Making of Consciousness. London: Vintage.

Danziger, K. (1990). Constructing the Subject: Historical Origins of Psychological Research. Cambridge: Cambridge University Press.

Danziger, K. (1997). Naming the Mind: How Psychology Found Its Language. London: Sage.

Dencik, L., Jørgensen, P. S., \& Sommer, D. (2008). Familie og børn i en opbrudstid. København: Hans Reitzels Forlag.

Descartes, R. (2002). Meditationer over den første filosofi. København: Samlerens bogklub.

Dewey, J. (1896). The reflex arc concept in psychology. The Psychological Review, 3, 357-370.

Dewey, J. (1916). Demokrati og uddannelse. (Denne udgave 2005). Århus: Klim.

Dewey, J. (1917). The need for social psychology. In J. A. Boydston (Ed.), The Middle Works, Volume 10: 1916-1917. (This edition published 1980). Carbondale, IL: Southern Illinois University Press.

Dewey, J. (1922). Human Nature and Conduct: An Introduction to Social Psychology. (This edition published 1930). New York: The Modern Library.

Dewey, J. (1925). Experience and Nature. Chicago, IL: Open Court.

Dewey, J. (1934). Art as Experience. (Late Works of John Dewey). Carbondale, IL: Southern Illinois University Press.

Dilthey, W. (1977). Descriptive Psychology and Historical Understanding. (First published 1894). The Hague: Martinus Nijhoff.

Dreyfus, H. (1991). Being-in-the-World - A Commentary on Heidegger's Being and Time, Division I. Cambridge, MA: The MIT Press.

Dreyfus, H. \& Taylor, C. (2015). Retrieving Realism. Cambridge, MA: Harvard University Press.

Edelman, G. \& Tononi, G. (2000). A Universe of Consciousness: How Matter Becomes Imagination. New York: Basic Books.

Foucault, M. (1993). About the beginning of the hermeneutics of the self: Two lectures at Dartmouth. Political Theory, 21, 198-227.

Foucault, M. (1994). The subject and power. In J. D. Faubion (Ed.), Power: Essential Works of Michel Foucault, Volume 3. London: Penguin.

Frances, A. (2013). Saving Normal. New York: HarperCollins.

Frank, L. (2007). Den femte revolution: Fortollinger fra hjernens tidsalder. København: Gyldendal.

Gadamer, H. G. (1960). Truth and Method. (2nd revised edition published 2000). New York: Continuum.

Gannik, D. (2005). Social sygdomsteori: Et situationelt perspektiv. Frederiksberg: Samfundslitteratur.

Gardner, H. (1987). The Mind's New Science. (2nd edition with a new epilogue). New York: Basic Books.

Gazzaniga, M. \& Heatherton, T. (2003). Psychological Science: Mind, Brain and Behavior. New York: Norton.

Gergen, K. (1994). Realities and Relationships. Cambridge, MA: Harvard University Press. Gerlach, C. \& Starrfelt, R. (2007). Hjernen og psyken. In B. Karpatschof \& B. Katzenelson (Eds.), Klassisk og modern psykologisk teori. København: Hans Reitzels Forlag.

Gibson, J. J. (1986). The Ecological Approach to Visual Perception. (First published 1979). Hillsdale, NJ: Lawrence Erlbaum Associates. 
Gillespie, A., Best, C., \& O’Neill, B. (2012). Cognitive function and assistive technology for cognition: A systematic review. Journal of the International Neuropsychological Society, 18, 1-19.

Giorgi, A. \& Giorgi, B. (2003). The descriptive phenomenological psychological method. In P. M. Camic, J. E. Rhodes, \& L. Yardley (Eds.), Qualitative Research in Psychology: Expanding Perspectives in Methodology and Design. Washington, DC: American Psychological Association.

Hacker, P. M. S. (2007). Human Nature: The Categorial Framework. Oxford: Blackwell.

Hacking, I. (1986). Making up people. In T. C. Heller, M. Sosna, \& D. Wellbery (Eds.), Reconstructing Individualism. Stanford, CA: Stanford University Press.

Hacking, I. (1995). Rewriting the Soul. Princeton, NJ: Princeton University Press.

Hacking, I. (1998). Mad Travelers: Reflections on the Reality of Transient Mental Disease. Charlottesville, VA: University Press of Virginia.

Hamilton, R. (2008). The Darwinian cage. Theory, Culture \& Society, 25, 105-125.

Harré, R. (1983). Personal Being. Oxford: Basil Blackwell.

Harré, R. (1997). Forward to Aristotle: The case for a hybrid ontology. Journal for the Theory of Social Behaviour, 27, 173-191.

Harré, R. (1998). The Singular Self: An Introduction to the Psychology of Personhood. London: Sage.

Harré, R. (2002). Cognitive Science: A Philosophical Introduction. London: Sage.

Harré, R. (2012). The brain can be thought of as a tool. Integrative Psychological and Behavioral Science, 46, 387-395.

Harré, R. (2016). Persons as the products and the producers of social acts. Culture \& Psychology, 22, 534-545.

Harré, R. \& Moghaddam, F. M. (2012). Psychology for the Third Millennium: Integrating Cultural and Neuroscience Perspectives. Thousand Oaks, CA: Sage.

Harré, R. \& Tissaw, M. (2005). Wittgenstein and Psychology: A Practical Guide. Aldershot: Ashgate.

Hauser, M. D. (2006). Moral Minds: How Nature Designed Our Universal Sense of Right and Wrong. New York: HarperCollins.

Healy, D. (2012). Pharmageddon. Berkeley, CA: University of California Press.

Heidegger, M. (1927). Being and Time. (This edition published 1962). New York: HarperCollins Publishers.

Heine, S. O. (2001). Self as a cultural product: An examination of East Asian and North American selves. Journal of Personality, 69, 881-906.

Holiday, A. (1988). Moral Powers: Normative Necessity in Language and History. London: Routledge.

Horwitz, A. V. (2002). Creating Mental Illness. Chicago, IL: University of Chicago Press.

Horwitz, A. V. \& Wakefield, J. C. (2007). The Loss of Sadness: How Psychiatry Transformed Normal Sorrow Into Depressive Disorder. Oxford: Oxford University Press.

Hume, D. (1978). A Treatise of Human Nature: Being an Attempt to Introduce the Experimental Method of Reasoning Into Moral Subjects. (First published 1739). Oxford: Clarendon Press.

Ingold, T. (2011). Being Alive: Essays on Movement, Knowledge and Description. London: Routledge.

Johnson, M. (2007). The Meaning of the Body: Aesthetics of Human Understanding. Chicago, IL: The University of Chicago Press. 
Katzenelson, B. (2007). Evolutionspsykologi. In B. Karpatschof \& B. Katzenelson (Eds.), Klassisk og moderne psykologisk teori. København: Hans Reitzels Forlag.

Kawamura, S. (1959). The process of sub-culture propagation among Japanese macaques. Primates, 2, 43-60.

Kessen, W. \& Cahan, E. D. (1986). A century of psychology: From subject to object to agent. American Scientist, 74, 640-649.

Kincaid, H. \& Sullivan, J. (2014). Classifying psychopathology: Mental kinds and natural kinds. In H. Kincaid \& J. Sullivan (Eds.), Classifying Psychopathology: Mental Kinds and Natural Kinds (pp. 1-10). Cambridge, MA: MIT Press.

Köhler, W. (1959). The Place of Value in a World of Facts. (First published 1938). New York: Meridian Books.

Krøjgaard, P. (2007). Spædbarnet. In B. Karpatschof \& B. Katzenelson (Eds.), Klassisk og moderne psykologisk teori. København: Hans Reitzels Forlag.

Krueger, J. W. (2008). Levinasian reflections on somaticity and the ethical self. Inquiry, $51,603-626$.

Lakoff, G. \& Johnson, M. (1980). Metaphors We Live By. Chicago, IL: University of Chicago Press.

Lakoff, G. \& Johnson, M. (1999). Philosophy in the Flesh. New York: Basic Books.

Latour, B. (1996). On interobjectivity. Mind, Culture, and Activity, 3, 228-245.

Latour, B. (2005). Reassembling the Social. Oxford: Oxford University Press.

Lave, J. (1993). The practice of learning. In S. Chaiklin \& J. Lave (Eds.), Understanding Practice: Perspectives on Activity and Context. Cambridge: Cambridge University Press.

Leontjev, A. N. (2002). Virksomhed, bevidsthed, personlighed. København: Hans Reitzels Forlag.

Louch, A. R. (1966). Explanation and Human Action. (This edition published 2000). Christchurch, New Zealand: Cybereditions Corporation.

MacIntyre, A. (1988). Whose Justice? Which Rationality? London: Duckworth.

Maiese, M. (2012). Rethinking attention deficit hyperactivity disorder. Philosophical Psychology, 25, 893-916.

Mammen, J. \& Mironenko, I. (2015). Activity theories and the ontology of psychology: Learning from Danish and Russian experiences. Integrative Psychological and Behavioral Science, 49, 681-713.

Martin, E. (1999). Mind-body problems. American Ethnologist, 27, 569-590.

Martin, E. (2007). Bipolar Expeditions: Mania and Depression in American Culture. Princeton, NJ: Princeton University Press.

McDowell, J. (1998). Mind, Value, and Reality. Cambridge, MA: Harvard University Press.

Meltzoff, A. \& Moore, K. (1997). Explaining facial imitation: A theoretical model. Developmental Psychology, 6, 179-192.

Merleau-Ponty, M. (1945). Phenomenology of Perception. (This edition published 2002). London: Routledge.

Mill, J. S. (1843). The Logic of the Moral Sciences. (This edition published 1987). London: Duckworth.

Mol, A. (2002). The Body Multiple: Ontology in Medical Practice. Durham, NC: Duke University Press.

Mol, A. (2008). I eat an apple. On theorizing subjectivity. Subjectivity, 22, 28-37.

Much, N. \& Harré, R. (1994). How psychologies "secrete" moralities. New Ideas in Psychology, 12, 291-321. 
Murray, L. \& Trevarthen, C. (1985). Emotional regulation of interactions between twomonth-olds and their mothers. In T. M. Field \& N. A. Fox (Eds.), Social Perception in Infants (pp. 101-125). Norwood: Ablex.

Nagel, T. (1974). What is it like to be a bat? The Philosophical Review, 83, 435-450.

Nielsen, M. (2016). My ADHD and me: Identifying with and distancing from ADHD. Nordic Psychology, 69, 33-46.

Nielsen, M. (2017). ADHD and temporality: A desynchronized way of being in the world. Medical Anthropology, 36, 260-272.

Noë, A. (2009). Out of Our Heads: Why Your Are Not Your Brain, and Other Lessons From the Biology of Consciousness. New York: Hill \& Wang.

Paterson, M. (2006). Consumption and Everyday Life. London: Routledge.

Pinker, S. (1999). How the Mind Works. London: Penguin.

Place, U. T. (1956). Is consciousness a brain process? In C. V. Borst (Ed.), The Mind-Brain Identity Theory. (Denne udgave 1970). London: Macmillan.

Proudfoot, D. (1997). On Wittgenstein on cognitive science. Philosophy, 72, 189-217.

Putnam, H. (1975). The meaning of "meaning". In Mind, Language and Reality: Philosophical Papers, Volume 2. Cambridge: Cambridge University Press.

Putnam, H. (1999). The Threefold Cord: Mind, Body, and World. New York: Columbia University Press.

Reckwitz, A. (2002). Toward a theory of social practices: A development in culturalist theorizing. European Journal of Social Theory, 5, 243-263.

Robinson, D. N. (1989). Aristotle's Psychology. New York: Columbia University Press.

Robinson, D. N. (2008). Consciousness and Mental Life. New York: Columbia University Press.

Rorty, R. (2004). The brain as hardware, culture as software. Inquiry, 47, 219-235.

Rorty, R. (1980). Philosophy and the Mirror of Nature. Princeton, NJ: Princeton University Press.

Rose, N. (1996). Inventing Our Selves: Psychology, Power, and Personhood. Cambridge: Cambridge University Press.

Rose, N. (1999). Governing the Soul: The Shaping of the Private Self. (2nd edition). London: Free Association Books.

Rose, N. (2009). Livets politik: Biomedicin, magt og subjektivitet i det 21. århundrede. København: Dansk Psykologisk Forlag.

Rose, N. \& Abi-Rached, J. M. (2013). Neuro: The New Brain Sciences and the Management of the Mind. Princeton, NJ: Princeton University Press.

Rosenberg, C. E. (2007). Our Present Complaint: American Medicine, Then and Now. Baltimore, MD: Johns Hopkins University Press.

Rouse, J. (2007). Social practices and normativity. Philosophy of the Social Sciences, 37, $46-56$.

Ryle, G. (1949). The Concept of Mind. London: Hutchinson \& Co.

Schatzki, T. R. (2001). Practice theory. In T. R. Schatzki, K. Knoor Cetina, \& E. von Savigny (Eds.), The Practice Turn in Contemporary Theory. London: Routledge.

Searle, J. (1980). Minds, brains and programs. Behavioral and Brain Sciences, 13, 585-642.

Searle, J. (1992). The Rediscovery of the Mind. Cambridge, MA: MIT Press.

Shweder, R. A. (1990a). Cultural psychology - What is it? In J. W. Stigler, R. A. Shweder, \& G. Herdt (Eds.), Cultural Psychology: Essays on Comparative Human Development. Cambridge: Cambridge University Press.

Shweder, R. A. (1990b). In defense of moral realism: Reply to Gabennesch. Child Development, 61, 2060-2067. 
Shweder, R. A., Much, N., Mahapatra, M., \& Park, L. (1997). The "big three" of morality (autonomy, community, divinity) and the "big three" Explanations of suffering. In A. M. Brandt \& P. Rozin (Eds.), Morality and Health. London: Routledge.

Singh, I. \& Rose, N. (2009). Biomarkers in psychiatry. Nature, 460, 202-207.

Smedslund, J. (2008). Har du sluttet å slå din kone? Ja/Nei. Kan psykologisk praksis være evidensbasert? Ja/Nei. Tidsskrift for Norsk Psykologforening, 45, 455-459.

Smith, B. H. (2005). Scandalous Knowledge: Science, Truth and the Human. Durham, NC: Duke University Press.

Smith, M. (2012). Hyperactive: The Controversial History of ADHD. London: Reaktion Books.

Spear, J. H. (2007). Prominent schools or other active specialties? A fresh look at some trends in psychology. Review of General Psychology, 11, 363-380.

Sprague, E. (1999). Persons and Their Minds. Boulder, CO: Westview.

Sterelny, K. (2012). The Evolved Apprentice: How Evolution Made Humans Unique. Cambridge, MA: MIT Press.

Still, A. \& Good, J. (1998). The ontology of mutualism. Ecological Psychology, 10, 39-63.

Taylor, C. (1989). Sources of the Self. Cambridge: Cambridge University Press.

Tolman, C. W. (2003). The moral dimension of psychological practice, theory, and subject matter. In D. B. Hill \& M. J. Kral (Eds.), About Psychology: Essays at the Crossroads of History, Theory, and Philosophy. Albany, NY: State University of New York Press.

Trevarthen, C. (1993). The self born in intersubjectivity: The psychology of an infant communicating. In U. Neisser (Ed.), The Perceived Self. Cambridge: Cambridge University Press.

Valsiner, J. (1987). Culture and the Development of Children's Actions. New York: John Wiley \& Sons.

Valsiner, J. (2007). Culture in Minds and Societies: Foundations of Cultural Psychology. New Delhi: Sage.

Valsiner, J. (2014). An Invitation to Cultural Psychology. London: Sage.

Valsiner, J. \& Rosa, A. (2007). The Cambridge Handbook of Sociocultural Psychology. Cambridge: Cambridge University Press.

Vetlesen, A. J. \& Stänicke, E. (1999). Fra hermeneutikk til psykoanalyse. Oslo: ad Notam, Gyldendal.

Vygotsky, L. S. (1927). The historical meaning of the crisis in psychology. Tilgængelig på www.marxists.org/archive/vygotsky/works/crisis/.

Vygotsky, L. S. (1929). Collected Works of L.S. Vygotsky. Volume 2: The Fundamentals of Defectology. (This edition 1993). New York: Plenum Press.

Vygotsky, L. S. (1978). Mind in Society: The Development of Higher Psychological Processes. Cambridge, MA: Harvard University Press.

Vygotsky, L. S. (1997). Research method. In R. W. Rieber (Ed.), The Collected Works of L.S. Vygotsky - Volume 4. New York: Plenum Press.

Watson, R. \& Coulter, J. (2008). The debate over cognitivism. Theory, Culture \& Society, $25,1-17$.

Wittgenstein, L. (1953). Philosophical Investigations. Oxford: Basil Blackwell.

Young, I. M. (1980). Throwing like a girl: A phenomenology of feminine body comportment, motility and spatiality. Human Studies, 3, 137-156.

Zittoun, T. (2006). Transitions: Development Through Symbolic Resources. Greenwich: Information Age Publishing. 
$\because$ Taylor \& Francis

Taylor \& Francis Group

http://taylorandfrancis.com 


\section{Index}

Abi-Rached, J. M. 56, 119

actions $82,83,85$

action theory 82

active externalism 97-9

activity theory $81-2$

anger $22-4,64$

Aquinas, T. 21-2

Aristotle 10, 11, 19-22, 24, 27, 52, 64, 75

artefacts 78

Asherson, P. 119

attention-deficit/hyperactivity disorder

(ADHD): essentialist and constructionist

approaches to 119-22; mediated mind

and 124-6; situational approach to $122-3$;

Tom's story 117-19

Augustine of Hippo, Saint 14, 21

autonomy 88

background 69-71

Barkley, R. 120

behaviourism 19, 49

Bem, S. 40-1, 44, 53

Bennett, M. R. 17, 24, 26, 38, 39, 42, 52

Berkeley, G. 17-18

Berk, L. 90, 92

Best, C. 123

biological body $59-60$

Blakemore, C. 38

Bloom, F. E. 36

body: as biological organism 59-60; cultural 61-2; dualism of body-mind 13-17, 58, 62-4, 71-3; ecological 60; habits as background 69-71; "image schemas" 67; metaphors 65-9; phenomenology of $60-4$; social 61

Bourdieu, P. 61

Bowden, G. 120

brain: cerebellum 36 ; cerebrum 36 ; hemispheres 37-8; materialistic perspectives on 39-51; as organ $6,36-9$; structures of $36-8$

brain research 2, 34-5

Brave New World (Huxley) 91

Brinkmann, S. 2, 11, 22, 25, 28, 30, 38, 43, 50, 59, 66, 67, 70, 89, 106, 117

Brown, T. 120

Buddhism 86-7

Buitelaar, J. 119

Buss, D. 53

Cahan, E. D. 28, 29

Cambridge Handbook of Sociocultural Psychology, The (Valsiner \& Rosa) 116

Cartesian materialists . 40

cerebellum 36

cerebral localization 37

cerebrum 36

Chaiklin, S. 78, 81, 82

Chalmers, D. 95, 97

children 5, 72-3, 79-80, 92-3, 105

Chinese room experiment 50

Chomsky, N. 49

Christianity 2, 11, 21, 58, 86-7, 105

Church, J. 121

Churchland, P. M. 42

Churchland, P. S. 42

Clark, A. 95, 97

cognition 50-1

cognitive neuroscience 45

cognitive science $3,39,42,44-51,53-4$, 59, 94, 108

Cognitive Science (Harré) 108

Cole, M. 77-8, 116

community 88

consciousness 9-10, 13-19, 52, 64

Copernicus 34

Cosmides, L. 53

Costall, A. 54 
Coulter, J. 3, 4, 24, 55

Crick, F. 34, 38, 46-7

cross-cultural psychology 87

cultural body $61-2$

cultural codes $87-8$

cultural-historical psychology 78

cultural psychology 87

culture 78, 86-90

Damasio, A. 38, 46

Danziger, K. 11, 12, 87, 102

Darwin, C. 19, 34, 55

Davidson, D. 27

De Anima (Aristotle) 20

Dencik, L. 91, 92

Dennett, D. 27

Descartes, R. 3, 13-19, 24, 27, 39, 52, 58, 78,94

developmental psychology 71-3

Dewey, J. viii, 4, 24, 26, 30-1, 50, 68, 70-1, 80, 96

Dilthey, W. 28, 29, 30

Dreyfus, H. 62, 63, 70

dyad 72

ecological body 60

ecological psychology 51

Edelman, G. 36, 38

Einstein, A. 82

eliminative materialism 9, 42-4

empathy 72-3

essentialism 119-22

ethnocentricity 89

ethology 28-9

evolutionary psychology $53-5$

existence 11-12

family 90-3

Fifth Revolution, The (Den femte revolution) (Frank) 34

Fodor, J. 27

folk psychology 43, 44, 90

Foucault, M. 102-3, 104

Frances, A. 125

Frank, L. 34-5, 56-7

Freud, S. 8, 34

functionalism 19, 44-6

Gadamer, H-G. 76

Galileo 21

Gall, F. J. 37

Gannik, D. 122-4

Gardner, H. 3, 45
Garfinkel, H. 55

Gates, B. 65

Gergen, K. 121

Gerlach, C. 37

Gibson, J. 63

Gillespie, A. 123

Good, J. 62

grammar: concept of 15-16; language instinct 49; M-grammar 110-12;

O-grammar 109-10; P-grammar 109; S-grammar 108

habits 69-71

Hacker, P. 4, 13, 17, 23, 24, 26, 38, 39 , 42,52

Hacking, I. 103, 119

Hamilton, R. 47, 54

Harré, R. viii, 4, 6, 24, 86, 107-9, 112, 114,127

Hauser, M. D. 49

Hegel, G.W.F. 75, 77

Heidegger, M. 20

hemispheres 37-8

Holiday, A. 89-90

Horwitz, A. V. 120

human sciences 28

Hume, D. 12, 17, 18, 21, 27, 28, 78

Husserl, E. 62

Huxley, A. 91

hybrid psychology 112

idealism 17-18

ideas $13-14,17-18$

infants 72

Ingold, T. 54

intentionality $5,63,79$

intentional world 83-4

intersubjectivity $72-3$

Johnson, M. 58, 59-62, 65-9

Jørgensen, P. S. 91, 92

Kahneman, D. 45

Kan, C. C. 119

Kant, I. 17, 18, 78, 81

Katzenelson, B. 53

Kawamura, S. 75

Kessen, W. 29

Kierkegaard, S. 75

Kosslyn, S. 45

Krøjgaard, P. 73

Krueger, J. W. 60

Kvale, S. 30 
Lakoff, G. 65

language $15-16,47,49,76,78,81,108-12$

language games viii

Latour, B. 77, 100, 104

Lazerson, A. 36

Leontiev, A. N. 82

Locke, J. 27

Looren de Jong, H. 40-1, 44, 53

Louch, A. R. 85

McDowell, J. 22

MacIntyre, A. 86

Mahapatra, M. 88, 89

Maiese, M. 120, 124

Mammen, J. 81

Martin, E. 56-7

Marx, K. 55, 76, 77, 89

materialist identity theory $19,40-1$

material resources 102-4

meaning 64-5, 69, 73, 96-9

Meaning of the Body, The (Johnson) 65

Meltzoff, A. 72

mental acts 68-9

mental development 93, 104-6

mental disorder: essentialist and constructionist approaches to 119-22; mediated mind and 124-6; situational approach to $122-3$

mental functions $27,31,36-9,49-51,64$, 78-81, 105, 111-12

mentalism 3, 31

mental modules 53-4

mental representations 13, 22, 32, 46-51, 64 mental rotation 97

mental technology 95-9

mereological fallacies 38-9, 47

Merleau-Ponty, M. 4, 6, 59, 60, 62-4

metaphysics 15, 19-20, 22, 86, 90

Mill, J. S. 28-9

mind: across cultures 86-90; brief scientific history 27-31; as a concept 4-5, 8-9; as consciousness 13-19, 52; current discourse on 2; as disembodied object 2; dualism of body-mind 13-17, $58,62-4,71-3$; evolutionary psychology perspective 53-5; false portrayal of 2 ; in family $90-3$; future of $126-8$; main tracks in history of ideas about 12-26; materialistic perspectives on 39-51; mental development 93, 104-6; mentalistic approach to 3-4; mental technology 95-9; metaphor for 6; mind as verb $24-5$; neurologically based theories of 56-7; psychological realm 11-12; in public dimension 63-4; as rationality 19-26; reductionist approach 3-4; social practices and 82-5; social technology 99-102; syntactic-modular approach 49-50; technology of the self and 102-4; things and 94-106

mindism 26-7, 31, 40

Mindmetic 35

Moghaddam, F. M. 112

Mohammed, Prophet of Islam 88

Mol, A. $60,122,124$

molecule grammar (M-grammar) 110-12

Moore, K. 72

morality $86-7$

moral philosophy 85

Much, N. 86, 88, 89

Murray, L. 72

nativism 49

naturalism 78

natural order 88

natural science 29

neo-Darwinist Cartesian problem 54-5

neural Cartesians 40

neuro-marketing 35

Newton, I. 12, 18, 21, 28

Nielsen, M. 43, 124

Noë, A. 6,35

nomic view 22-4, 82-3

normative accountability $84-5$

normative view $22-4,82-3$

O’Neill, B. 123

operative intentionality 63

organism grammar (O-grammar) 109-10

Park, L. 88, 89

participants 78

Paterson, M. 62

person grammar (P-grammar) 109

personism 26-7, 31, 32

Phaedo (Plato) 13

phenomenological body $60-4$

phenomenology 62

Philosophical Investigations (Wittgenstein) 16

phobias 25-6

phrenology 37

physicalism 19

Pinker, S. 19, 45, 53

Place, U. T. 40

Plato 13-14, 19-20, 66

practical rationality 21 
practice $84-5$

pragmatic psychology 80

primary metaphors $67-8$

private language 47

Proudfoot, D. 47

psyche 10

psychological development $80-1$

psychological realm 11-12

psychology: crisis in 31 ; cross-cultural

87; cultural 86-90; cultural-historical

78-81; current discourse on mind 1-2;

dualization of 27-31; ecological 51;

evolutionary $53-5$; experimental 16 ; folk 43, 44, 90; mentalistic 3, 31; as moral science 28-9; pragmatic 80 ; principles for hybrid 112-14; qualitative 28, 30; quantitative 28,30 ; scientific 28 ; sociocultural 76-82; task-tool metaphor 112; taxonomic priority principle $113-14$; technology of the self and 102-4; twosided nature of 27-31

Putnam, H. 24, 96-7

qualia 9, 51-2

qualitative psychology 28,30

quantitative psychology 28,30

rationality 19-26

reasoning 24

Reckwitz, A. 84

reductionism 3, 31, 39

Robinson, D. N. 22, 39

Rorty, R. 31, 51

Rosa, A. 116

Rose, N. 11, 56-7, 102, 119, 128

Rosenberg, C. E. 120

Rouse, J. 84

Ryle, G. 27, 58

Schatzki, T. R. 84

scientific psychology 28

Searle, J. 27, 50, 70

Sharrock, W. 3, 4

Shweder, R. 29, 83-4, 87, 88, 89

signs 76

Singh, I. 119

Smith, B. H. 53

social body 61

social constructionist approaches 119-22

social gender inequality 61

social practices $82-5$

social sciences 28

social technology 99-102

society $75,77,81$

socio-cultural psychology 76-82 solipsism 14

Sommer, D. 91, 92

soul 11-12, 20

soul grammar (S-grammar) 108

Spear, J. H. 127

spirit 75-6

Sprague, E. 26, 31

Stänicke, E. 63

Starrfelt, R. 37

Sterelny, K. 63

Still, A. 62

suffering 89

symbolic resources 102-4

syntactic-modular approach 49-50

System of Logic, A (Mill) 28

Tanggaard, L. 25, 66

task-tool metaphor 112

taste 61-2

taxonomic priority principle 112

Taylor, C. 11, 62, 63, 85

technology: mental 95-9; of the self 102-3; social 99-102

teleology 111-12

theoretical rationality 21

Tolman, C. W. 89

Tononi, G. 36

Tooby, J. 53

Treatise of Human Nature, A (Hume) 18

Trevarthen, C. 72

Valsiner, J. ix, 4, 29, 76, 77, 91, 116

Vetlesen, A. J. 63

Vygotsky, L. 4, 30-1, 78-82

Wakefield, J. C. 120

Watson, J. 34

Watson, R. 55

White, S. H. 28

Winnicott, D. 72

Wittgenstein, L.: basic understanding of mind 22; concept of meaning 96; concept of mind 24-5; grammar 108; importance of 4; language games viii, 20; life-form concept $39,58,77$; mental representations 47-8, 69; moral values 89 ; personism and $26-7,32$; perspective on actions 83; Philosophical Investigations 16; on psychology's status as science 15-16

Wundt, W. 30

Young, I. M. 61

Zittoun, T. 105-6 